University of Wisconsin Milwaukee UWM Digital Commons

Theses and Dissertations

December 2018

\title{
A Biofuel Supply Chain Equilibrium Analysis with Subsidy Consideration
}

Amirsaman Hamzeh Bajgiran

University of Wisconsin-Milwaukee

Follow this and additional works at: https://dc.uwm.edu/etd

Part of the Industrial Engineering Commons

\section{Recommended Citation}

Hamzeh Bajgiran, Amirsaman, "A Biofuel Supply Chain Equilibrium Analysis with Subsidy Consideration" (2018). Theses and Dissertations. 1992.

https://dc.uwm.edu/etd/1992

This Dissertation is brought to you for free and open access by UWM Digital Commons. It has been accepted for inclusion in Theses and Dissertations by an authorized administrator of UWM Digital Commons. For more information, please contact open-access@uwm.edu. 


\title{
A BIOFUEL SUPPLY CHAIN EQUILIBRIUM ANALYSIS WITH SUBSIDY CONSIDERATION
}

by

Amirsaman Hamzeh Bajgiran

A Dissertation Submitted in

Partial Fulfillment of the

Requirements for the Degree of

\author{
Doctor of Philosophy \\ in Engineering
}

at

The University of Wisconsin-Milwaukee

December 2018 


\title{
ABSTRACT \\ A BIOFUEL SUPPLY CHAIN EQUILIBRIUM ANALYSIS WITH SUBSIDY CONSIDERATION
}

\author{
by \\ Amirsaman Hamzeh Bajgiran \\ The University of Wisconsin-Milwaukee \\ Under the Supervision of Professor Jaejin Jang
}

Growing environmental concerns in the last few decades along with the energy security issues have led governments to take actions to reduce their dependence on fossil fuels and enhance renewable energy usage, including biofuels. Enforcement of federal or state mandates and regulations that obligate a certain amount of biofuel production are one of the main levers that governments use to achieve their goals. In the U.S. as an example, Renewable Fuel Standard (RFS) requires the production of 36 billion gallons of ethanol by 2022, 21 billion gallons of which should be advanced biofuels derived from biostocks other than corn starch. In parallel with making regulations, governments also incentivize the biofuel producers and farmers who provide the input biomass by paying subsidies to promote the industry. In this research, one of our main goals is to study the government's role as both a regulator and incentive payer in the biofuel production industry.

In the first chapter we consider a pilot biofuel supply chain problem, in which a farmer supplies two downstream refineries with non-identical crops (corn and energy crop). The problem has been modeled as a multi-leader-single-follower game to derive the farmer's decisions on land allocation as well as refineries' proposed prices to the farmer for their raw materials. We consider subsidizing the farmer and the refinery that uses the energy crop to study whether a subsidy plan can enhance the advanced biofuel production and meet the existing mandate. We solve the problem under four cases based on the willingness of the farmer to sell corn to the food market as well as the availability of farming land expansion. The Nash Equilibrium 
(NE) is derived for all cases, and parametric analyses are used to study the effect of subsidies on the profit of the players and the total social welfare of the supply chain. We observe that a government's expenditure can be offset by the increase of the social welfare under certain circumstances. We find the minimum budget requirement to meet the EPA's mandate and show that a specific budget can be distributed to the farmer and the refineries in different ways while obtaining the same results.

In Chapter 3, we extend the above equilibrium analysis by relaxing a simplifying assumption regarding the capacity of the refineries, which assumes that the refineries can process any amount of crop from the land that the farmer may allocate to them. Relaxing this assumption gives us the possibility of analyzing the problem under more general circumstances, although the assumption may be acceptable in a relatively small regional scope. We obtain closed form solutions for the supply chain equilibrium under the existence of the new capacity constraints and find that refineries offer lower prices under the new condition, while their profit is larger. However, this is not the case for the farmer, and her profit diminishes under this capacitated problem.

In Chapter 4, we extend the scope of our problem by generalizing the model to consider multiple players and make conclusions more general. Our bilevel supply chain is modeled as an equilibrium problem with equilibrium constraints (EPEC) and solved using a linearization method and a commercial solver of GAMS. We perform several analyses including the effect of the farmer's cost structure and the number of the refineries on a few socio-economic measures. We also perform a sensitivity analysis on a few critical industry and market parameters. Through our analyses, we first find that the budget requirement to meet the government's goal is higher than what other similar works have estimated. Second, we find that as new refineries are built, the government should spend more on the subsidy to obtain the goal; however, the total social welfare increases because of an increase in the biofuel market consumer surplus from lower fuel price. We show that the government's expenditure will not be offset by the increase of the social welfare in this multi-player case as the required conditions found from Chapter 2 do not hold. Finally, we find that the advanced biofuel technology improvement is the most important factor among the considered industry 
related factors in reducing the subsidy requirements. Finally, in Chapter 5, we summarize the introduced models in previous chapters, the effect of the capacity constraint, and the effect of the competition. 
(C) Copyright by Amirsaman Hamzeh Bajgiran, 2018

All Rights Reserved 
To

My parents,

My wife,

And my sisters 


\section{TABLE OF CONTENTS}

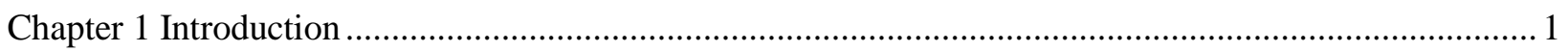

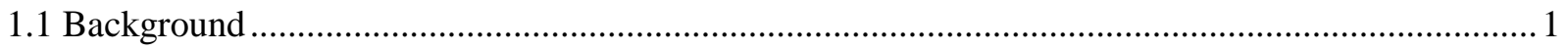

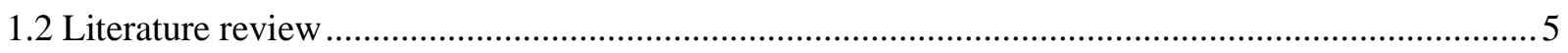

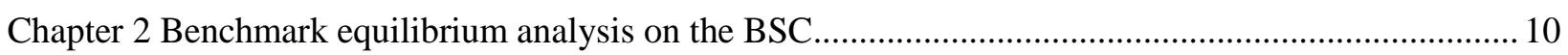

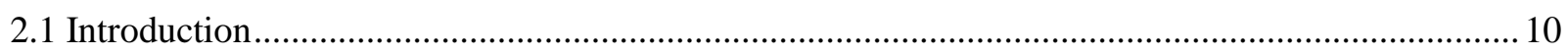

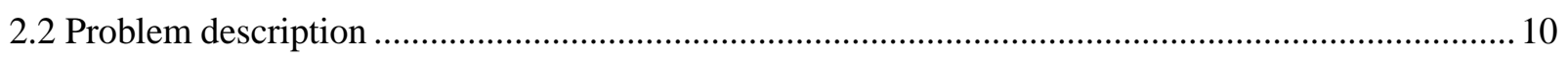

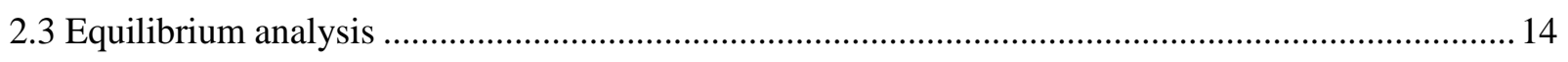

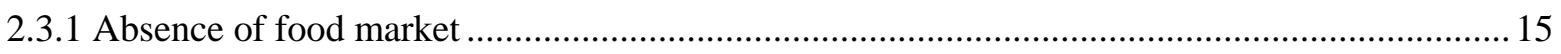

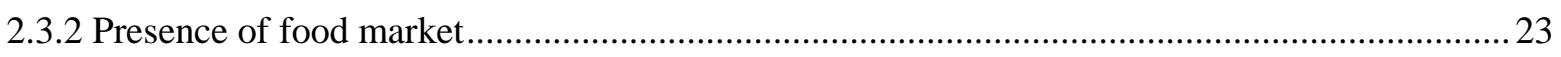

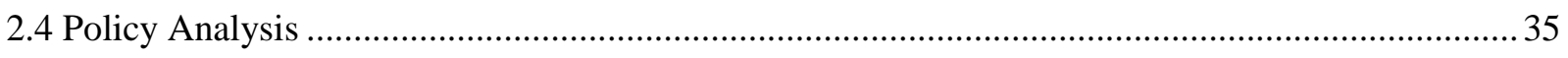

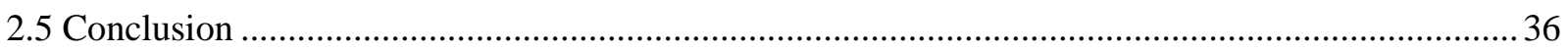

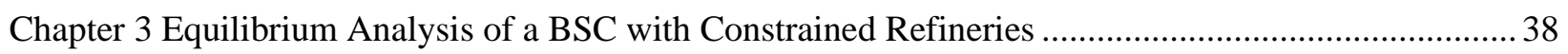

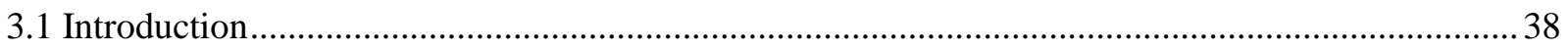

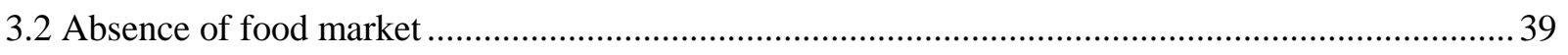

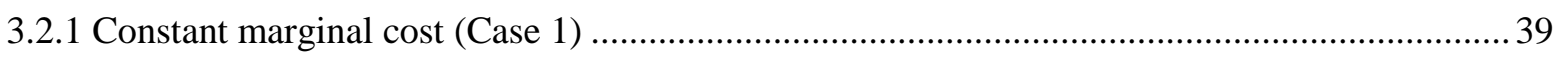

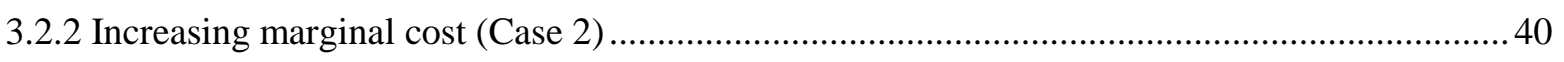

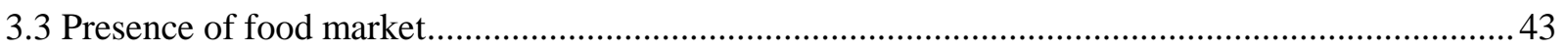

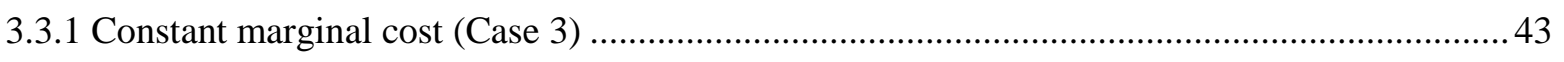

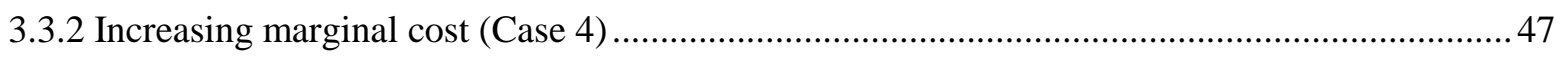

Chapter 4 Equilibrium analysis of a bilevel BSC with EPEC approach ................................................51

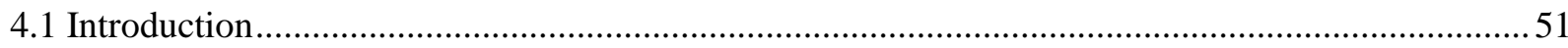

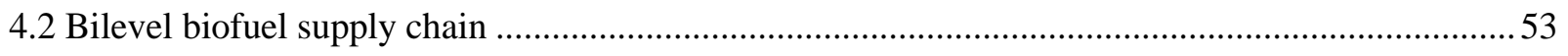

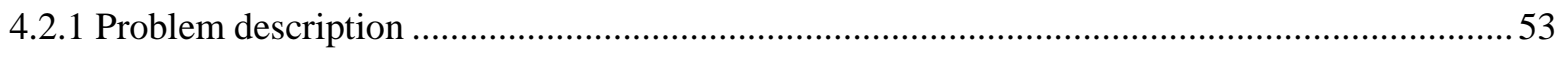

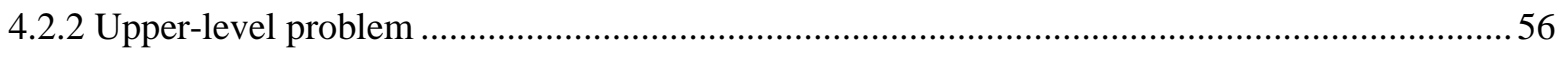

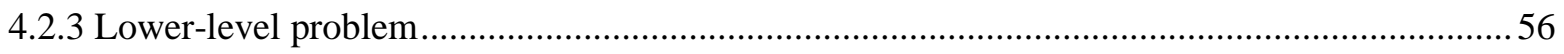

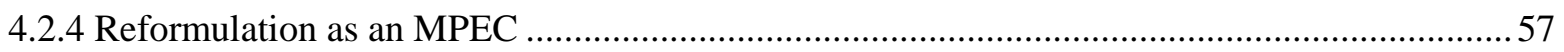

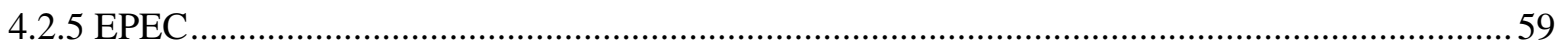

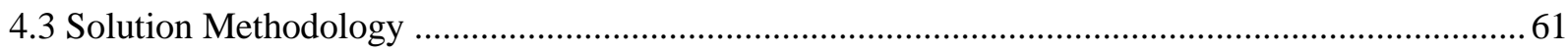

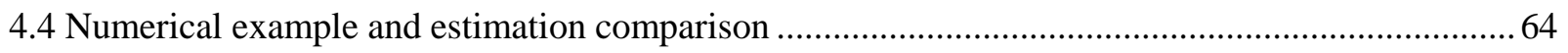

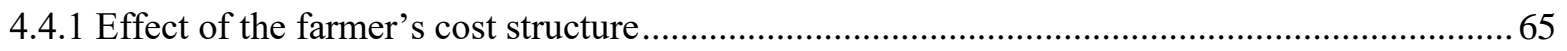

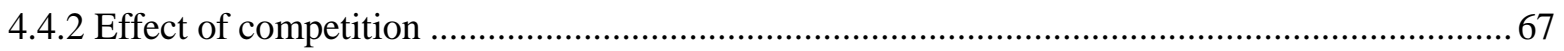

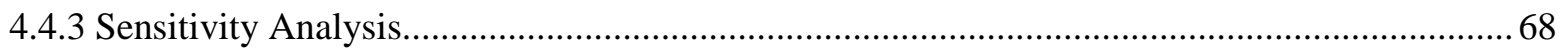

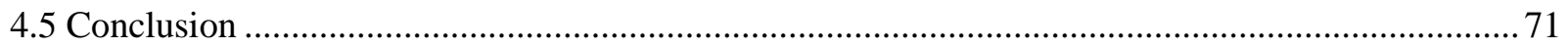




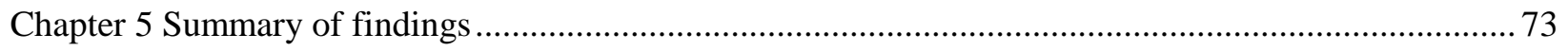

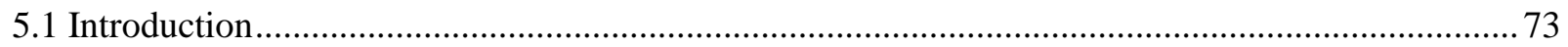

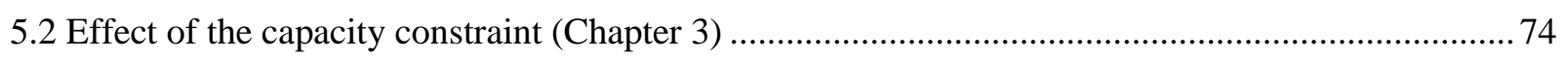

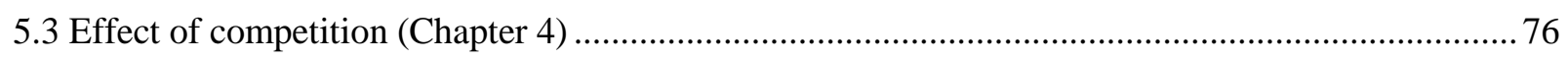

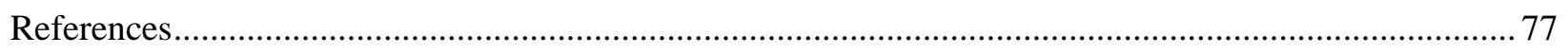

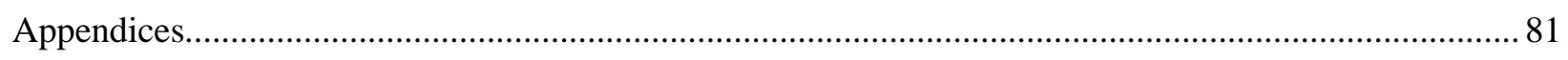

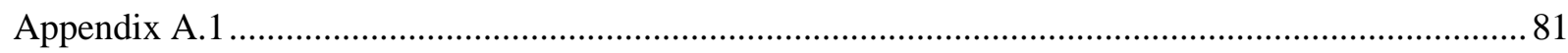

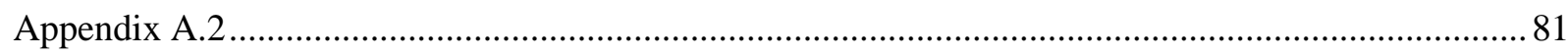

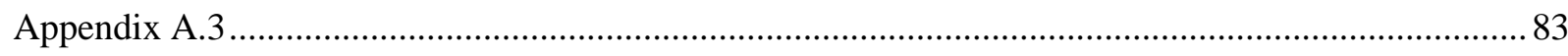

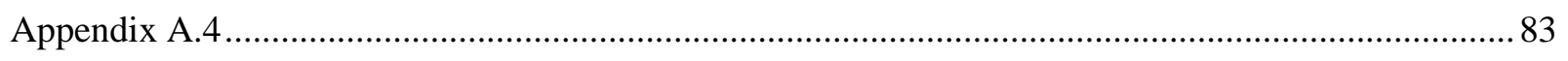

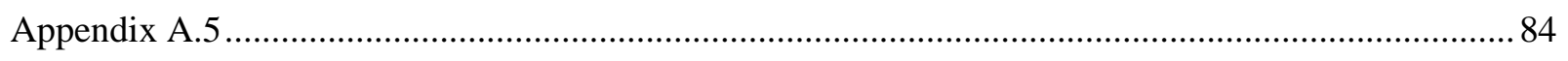

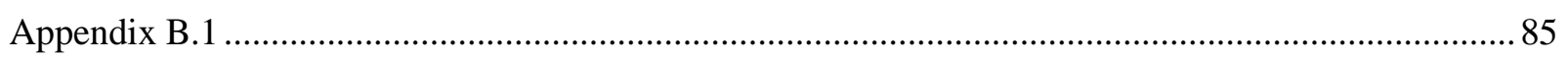

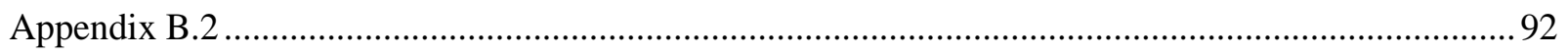

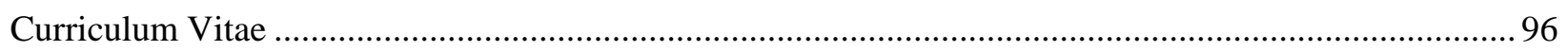




\section{LIST OF FIGURES}

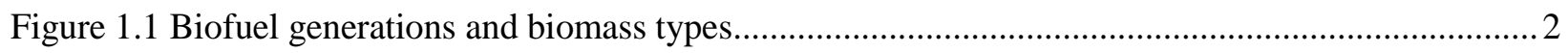

Figure 1.2 Biofuel production trend in recent years and projected year ................................................. 4

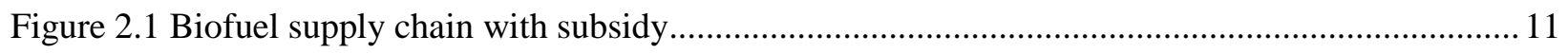

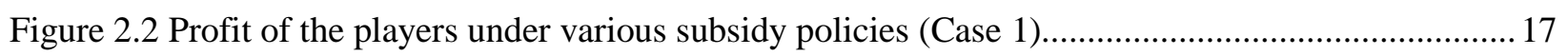

Figure 2.3 Total SW increase breakdown under various policies vs. total subsidy paid (Case 1)............. 18

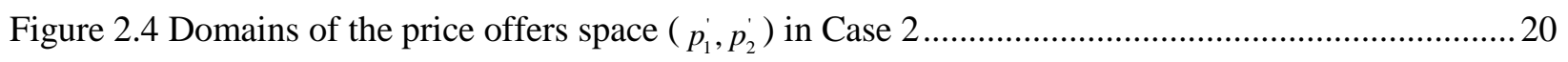

Figure 2.5 Profit of the farmer and refineries under various subsidy policies (Case 2)..........................23

Figure 2.6 Total SW increase breakdown under various policies vs. subsidy paid (Case 2)....................23

Figure 2.7 Domains of the price offers' space $\left(p_{1}^{\prime}, p_{2}^{\prime}\right)$ in Case 3 ...........................................................25

Figure 2.8 Profit of the farmer and refineries under various subsidy policies (Case 3)...........................28

Figure 2.9 Total SW increase breakdown under various policies vs. subsidy paid (Case 3)....................28

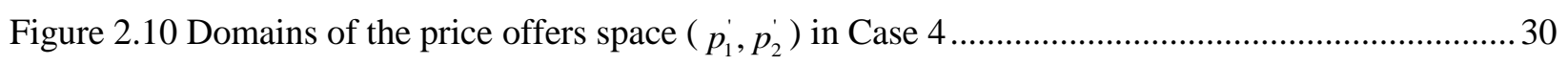

Figure 2.11 Profit of the farmer and refineries under various subsidy policies (Case 4).......................... 34

Figure 2.12 Total SW increase breakdown under various policies vs. subsidy paid (Case 4)................... 34

Figure 2.13 Best policy selection in Cases 2 (with corn market) and 4 (without corn market) .................36

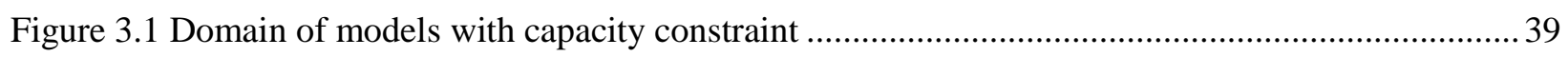

Figure 3.2 Total SW increase breakdown under various policies vs. subsidy paid (Capacitated model-Case

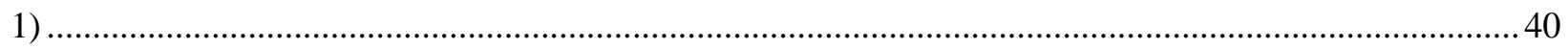

Figure 3.3 Domains of the price offers' space $\left(p_{1}^{\prime}, p_{2}^{\prime}\right)$ in Case 2 of the capacitated model .....................42

Figure 3.4 Domains of the price offers' space $\left(p_{1}^{\prime}, p_{2}^{\prime}\right)$ in Case 3 of the capacitated model .......................45

Figure 3.5 Domains of the price offers' space $\left(p_{1}^{\prime}, p_{2}^{\prime}\right)$ in Case 4 of the capacitated model ...................... 48

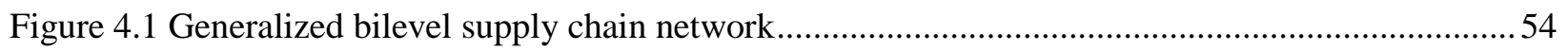

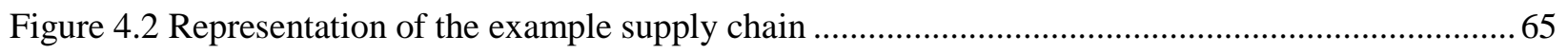

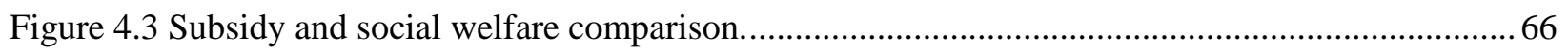

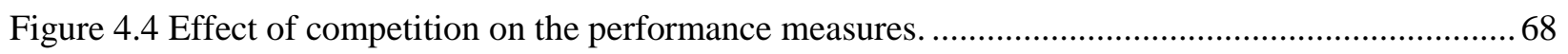

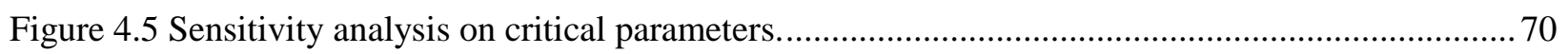

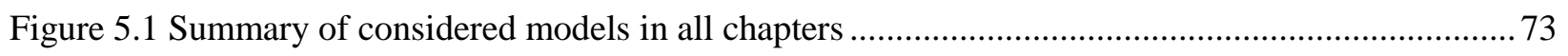

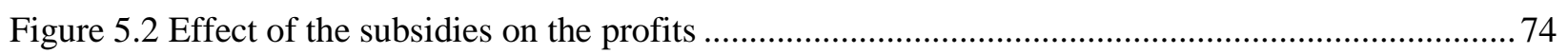

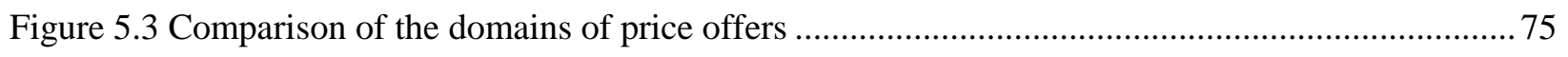

Figure 5.4 Comparison of results for Case 1 after adding capacity limit................................................ 76

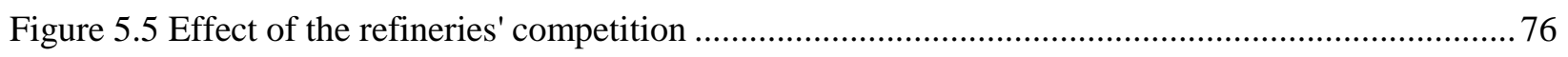




\section{LIST OF TABLES}

Table 1.1 Summary of the most relevant works …............................................................................

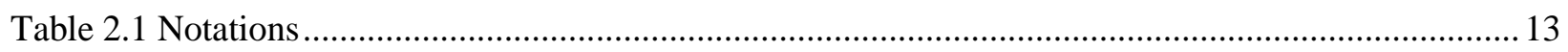

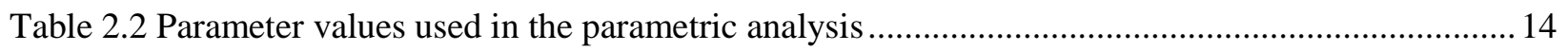

Table 2.3 Domains, conditions on offered prices and the farmer's BR in Case 2 ..................................20

Table 2.4 Domains, conditions on offered prices and the farmer's BR in Case 3..................................25

Table 2.5 Domains, conditions on offered prices and the farmer's BR in Case 4 ..................................... 30

Table 3.1 Conditions on offered prices and the farmer's BR in Case 2 of the capacitated model ..............42

Table 3.2 Conditions on offered prices and the farmer's BR in Case 3 of the capacitated model ..............46

Table 3.3 Conditions on offered prices and the farmer's BR in Case 4 of the capacitated model ............. 49

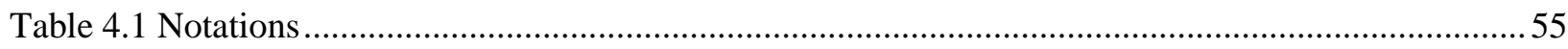

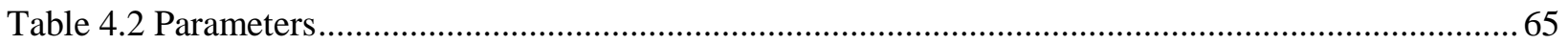




\section{ACKNOWLEDGEMENTS}

This dissertation would not have been possible without the support and help of many, a few of whom it is possible to give particular mention.

I would like to express the deepest appreciation to my advisor, Professor Jang, during my studies at University of Wisconsin-Milwaukee. He continually and convincingly conveyed a spirit of adventure in research, and excitement in teaching. Without his guidance and persistent help, this dissertation would not have been possible.

I would also like to express my sincere gratitude to my committee members, Professor Otieno, Professor Petering, Professor Fang, and Professor Peoples for their valuable comments and insight.

I would like to acknowledge the financial and academic support of the Department of Industrial and Manufacturing Engineering, the College of Engineering and Applied Sciences, and the Graduate School at University of Wisconsin-Milwaukee. Specially, the teaching assistantship appointments, graduate student and Chancellor awards gave me the necessary financial support during my academic persuits at UWM.

Above all, and most importantly, I would like to express my very profound gratitude to my family, especially my mother, who emphasized the value of education, guided me through life and served as my role model. Special thanks to my wife for her continued love and encouragement and providing me with unfailing support throughout my years of study and research. At the same time, I am grateful to my friends for their support, lasting interest and friendship even at times of hardship. This accomplishment would not have been possible without them. 


\section{Chapter 1 Introduction}

\subsection{Background}

Energy security and global warming are two strong driving forces that motivates governments and industry to invest in renewable energy research and production. Oil price fluctuations, coalitions of producers to keep the production level low, and the limited amount of oil available on the earth are a few economic drivers of that investment. On the other hand, growing environmental concerns for greenhouse gas (GHG) emissions in the last few decades have led governments to take actions to reduce the discharge rate of the pollution into natural resources. Enforcement of federal or state mandates and regulations that obligate a certain amount of renewable energy production are examples of such actions in the US to promote renewable energy research, development, and production. According to the Annual Energy Outlook 2016, the projected share of renewable energy from total energy production increases from $9 \%$ in 2015 to $15 \%$ in 2040, which includes liquid biofuels (Annual Energy Outlook, 2016). This increase would be a response to the above mentioned federal and state motivators. Renewable Fuel Standard (RFS) and its revision (RFS2) are examples of powerful energy regulatory drivers in biofuel production industry (EPA, 2007). The mandate requires the production of 36 billion gallons of ethanol by 2022, 21 billion gallons of which should be advanced biofuels derived from biostocks other than corn starch.

Biofuel production technology has evolved in the last few decades, and currently there exist four generations of biofuel depending on the technology and the input biomass (Figure 1.1). The production technology for the first three generations are well established and commercialized, while the fourth is still in a lab scale. First generation biofuels use arable products (starch-based) as input, and the choice of the crop depends on its abundance in the region (e.g. corn or sugar cane). Second, third, and fourth generation biofuels, also known as advanced biofuels, use non-starch-based biomass such as energy crops, agricultural 
residues, algae, and genetically modified grass. In this research, we consider cellulosic based energy crops (second generation) as the source biomass of advanced biofuel. Advanced biofuels are preferred over conventional (first generation) ones for a few important reasons. First, they don't interfere with food supply chain if their inputs are grown on marginal lands. Wu and Langpap (2015) show that the 2007 biofuel mandates have increased the price of corn by $25-40 \%$ and increased the price of food by $1.5-2.5 \%$. Second, they have the potential to reduce GHG emissions by $60 \%$ (EPA, 2007).

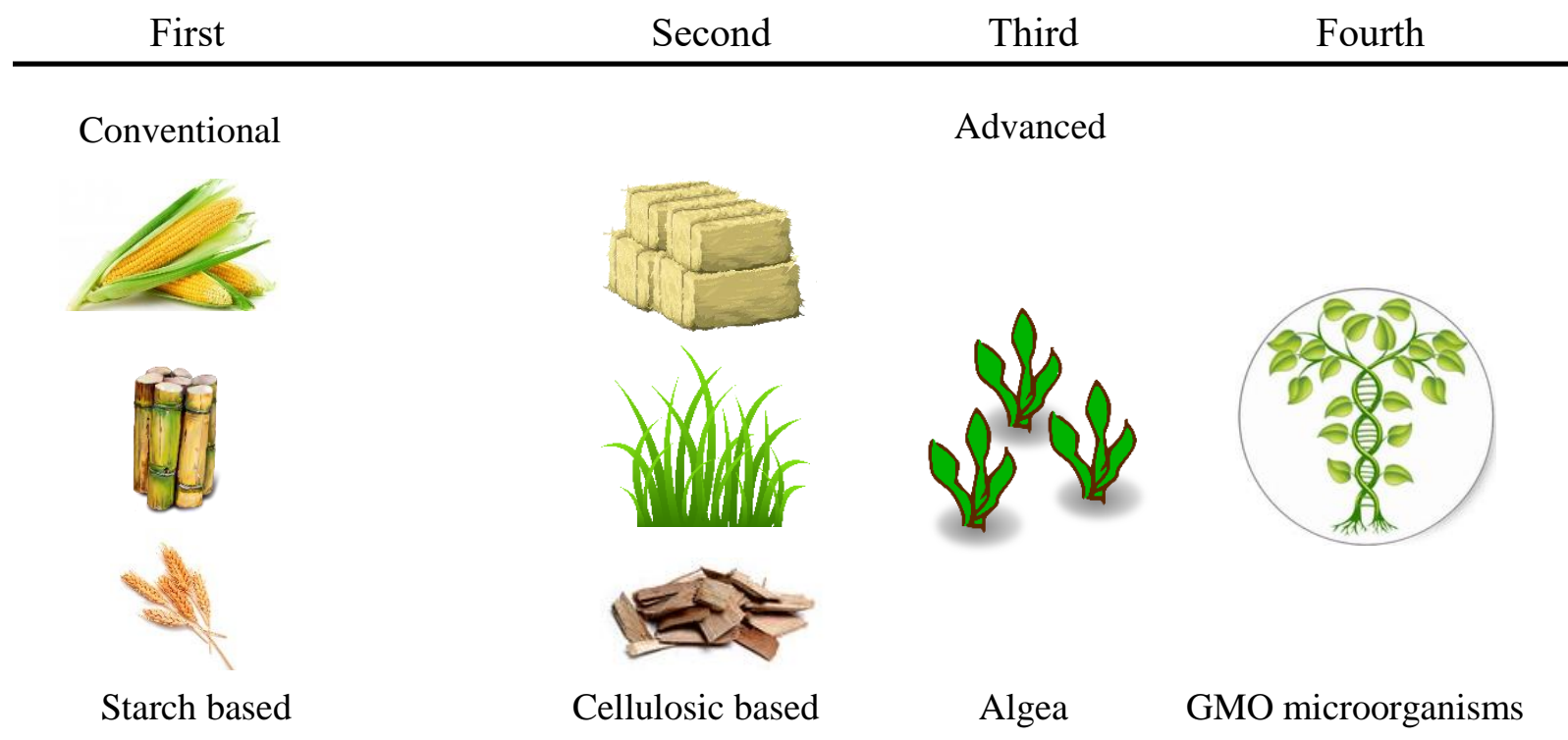

Figure 1.1 Biofuel generations and biomass types

The development of a refinery that produces advanced biofuel significantly influences the economy in the region if the refinery is fed with energy crops from local farms. Increased demand for such crops motivates farmers to change their choice of cultivation from corn (in the U.S. case, or sugar cane in Brazil) to these crops. As a result, the price and production quantity of crops can be affected. Advanced biofuel production is more expensive in many cases, and because of the lower energy content of some biostocks used for advanced biofuels, it makes them bulkier and their transportation costs higher. These drawbacks have prevented advanced biofuels from penetrating in the biofuel market in spite of their advantages over the 
first-generation biofuels. In a recent work, Li et al. (2015) study the profitability of investment in cellulosic biofuel industry in Iowa and conclude that, with current technology profiles, investment in the future is more profitable than now.

Current production trends (Figure 1.2) show that it is unlikely to reach the EPA's goal, which could be because of being less economically attractive for advanced biofuel producers (refineries). Financial barriers of investment in advanced biofuel production can be lifted if enough subsidies are provided for some firms in the supply chain. On one hand, refineries can be incentivized to overcome the low conversion rate and technology expenses of advanced biofuel production in various ways such as receiving a tax credit. On the other hand, farmers can also be incentivized to grow energy crops, and it can be done by creating markets for those crops or paying subsidies to them. The effectiveness of such incentivizing mechanisms on Biofuel Supply Chain (BSC) has not been addressed properly yet in the literature. In one of the most relevant works, Luo and Miller (2013) calculate the incentives required to stimulate the advanced biofuel production under a simplified framework, which does not consider the socio-economic impact of the biofuel supply chain. In this research, we consider a supplier (farmer) with possibly limited production capacity providing two different biostocks. The downstream manufacturers (refineries) with different technology profiles use these biostocks to make an identical final product (biofuel) while competing for the limited existing lands of the farmer. We investigate the new market equilibrium at the BSC, and its effect on the achievement of EPA's mandate when subsidies are given to the farmer and the advanced refineries. More specifically, we investigate the following questions:

1. How do firms in the BSC such as farmer, corn-based refinery, and energy crop-based refinery interact, and how their behavior is influenced by the subsidy policies?

2. What effects does the choice of farmer to expand her land have on the performance of the supply chain? 


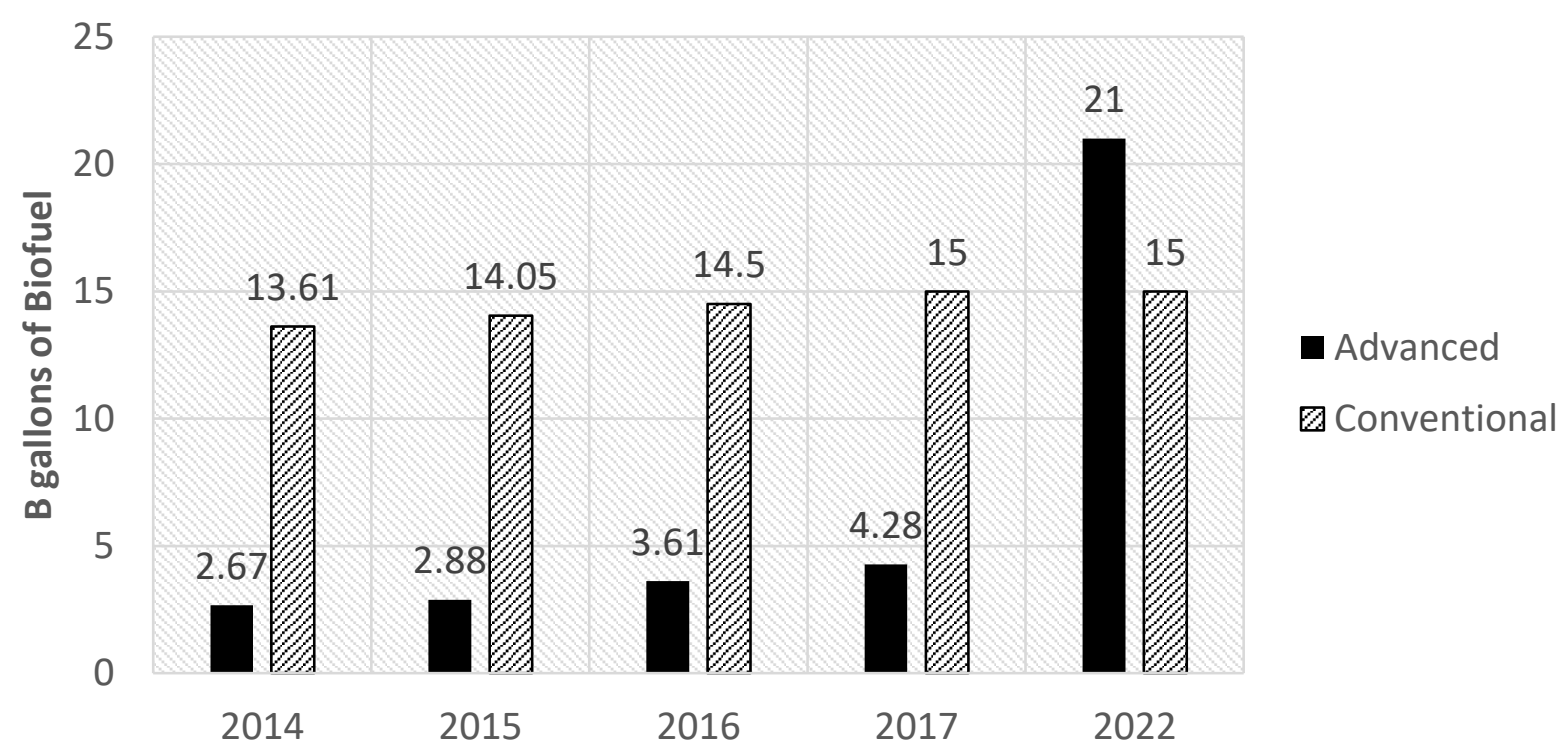

Figure 1.2 Biofuel production trend in recent years and projected year

3. Which firms are better off, and which are affected by the subsidy plans? Is the government expenditure for the subsidy plan offset by the increase of the social welfare? What subsidy plan has the lowest financial burden for the government while being effective enough?

4. What effects does the number of refineries who are competing for limited farming lands have on the performance of the supply chain?

The rest of the dissertation is organized as follows. Chapter two introduces the general framework of our biofuel supply chain, and the assumptions we have used followed by the notations. It is continued by the formulation of various cases that we have considered based on our assumptions and their analytical solution is presented in terms of several propositions. The equilibria are found using backward induction, and the marginal effect of subsidies on the equilibria are also studied. We also discuss the policy analysis in this chapter and draw important conclusions. Chapter three is an extension of Chapter two, in which we add an assumption about the capacity limit of the refineries and solve the model for all the cases considered in Chapter two. In Chapter 4, we move further and generalize our model to be able to consider any number of firms in the supply chain. The model is formulated as an equilibrium problem with equilibrium constrains (EPEC), which we solve for the solution, called Generalized Nash Equilibrium. A comprehensive analysis 
is performed using a numerical example and realistic parameter values to investigate the validity of conclusions in the first chapter and study the effect of the number of refineries on the supply chain's performance.

\subsection{Literature review}

Decision making in a supply chain can take place at any level of the hierarchy including strategic (longterm), tactical (medium- term), and operational (short-term). One of the main streams of research in BSC addresses the strategic level of decision makings such as finding the optimal location of facilities, transportation mode, required technology, and future development options. Earlier works at this level used GIS to optimize the location and transport decisions (e.g. Panichelli et al. 2008 and Zhang et al. 2011). However, mathematical programming has been more extensively used in recent studies (Xie et al. 2014; Akgul et al. 2012). Xie et al. (2014) studied the integration of multimodal transport into cellulosic biofuel supply chain and evaluated the cost-effectiveness of multimodal transport against single mode. Huang et al. (2014) addressed location and size decisions of refineries and fuel storages considering the seasonality of biomass production. Tactical and operational decisions such as land allocation, storage, transportation, and final product flow between nodes are another stream of articles in BSC (An et al. 2011; Marvin et al. 2011; Papapostolou et al. 2011; Wanga et al. 2013; Cobuloglu et al. 2015). We cannot draw a sharp line between these levels of decision making and there are several works that investigate all of them such as Bai et al. (2011), Huang et al. (2014), and Bai et al. (2012). However, our work can be considered at the tactical

level as we are determining quantity and pricing decisions, which are midterm decisions. It means that they are neither long term (e.g., capacity decision) nor short term (e.g., inventory decision).

Policy analysis on BSC has been done in recent years to evaluate its impacts on farm and renewable fuel industries (e.g. Wu and Langpap, 2015; Huang et al. 2014; Palak et al. 2014). Most of the works study existing mandates and subsidy plans concerning refineries only. For example, Chen et al. (2010) elaborate on the need to shift the subsidy plans from conventional biofuels toward advanced biofuels to prevent the competition for land use. They find that biofuel production would rely on corn at least for $50 \%$ to meet the 
RFS mandate without subsidies. It is claimed that with the existence of tax credit for cellulosic ethanol, its share would increase to $88 \%$ of the cumulative biofuels. That would reduce the competition for land as well as corn prices. The absence of subsidy for advanced biofuels would result in importing almost $10 \%$ of total biofuel requirement to make up the advanced biofuel share in the mandate (Nunez et al. 2013).

Incentivizing farmers is another aspect of subsidy plans to stimulate advanced biofuel production. Conservation Reserve Program (CRP) is one of the policies that directly aims farmers, and its impact on BSC has been studied previously (e.g. Ferris and Joshi 2010; Bai et al. 2012; Cobuloglu et al. 2015; Bai et al. 2016). However, subsidizing farmers to grow energy crops along with subsidizing refineries has not been clearly addressed, and in this research, we try to fill this gap. In a work by Luo and Miller (2013), they explore the efficacy of a potential carbon market as an incentive mechanism to encourage farmers and refineries to promote advanced biofuel production under two conservative and optimistic scenarios. They show that it is only under the optimistic technological scenario that the carbon market is effective. However, their work is restricted by several simplifying assumptions, which is a source of motivation for us to perform a more comprehensive research and policy analysis. The simplifying assumptions they have considered include a constant market price for the biofuel and ignoring the existence of food market to which farmers are capable to sell their product.

Several researchers have studied BSC optimization under a centralized framework (i.e. they assume that all operations are under the supervision of a corporation and the objective is to maximize its profit) or have optimized the objective function of a single firm in the supply chain (Del-Mas et al. 2011; Awudu and Zhang, 2013; Marufuzzaman et al. 2014). However, more often than not, farmers and refineries make decisions independently for their own benefits, and their objectives are usually conflicting. In this case, the decision of a firm in the supply chain or outside of the supply chain (e.g. government) interacts with the decision of the others and the resultant market conditions are determined by the decisions of the firms collectively. Game theoretic models can explain such environments. The way that players interact with each other defines the type of game, which can be classified under various schemes such as Cooperative/Non- 
cooperative or Simultaneous/Sequential (Yue et al. 2014). Most of the existing literature in BSC design focuses on a cooperative (centralized) system. For example, Zamarripa et al. (2013) in their cooperative scenario, consider a set of supply chains as a single firm and minimize the total cost. Yue and You (2014) develop a cooperative model assuming that there is no secret information among firms, and decision making is based on negotiation.

The non-cooperative aspect of the problem deserves as much attention as the cooperative framework since it can capture the competition of firms (e.g. farmers, refineries, blenders, fuel and food market, and government) in the supply chain. The firms are usually at different levels (echelons) of a BSC, and in case they make decisions sequentially, leader-follower (Stackelberg) game modeling could be beneficial. Bai et al. (2012) explore a problem, in which a corn-based refinery first proposes its price to farmers, and in response, farmers independently decide the quantity they give to the refinery and a food market considering the transportation costs. The model is designed as a Stackelberg game with the refinery as the leader and farmers as followers. Huang et al. (2014) consider a multi-echelon design (consisting of biomass producers, refineries, and blenders) for the supply chain. They solve the problem under two assumptions of perfect and imperfect competitions between blenders. In a perfect competition, blenders have no market power (i.e. they are price takers) while, in the imperfect competition, blenders offer their price for biofuel to refineries. In another work by Yue and You (2014), they design a non-cooperative supply chain, in which a single refinery makes strategic decisions first and followers (suppliers and customers) react to the leader's move to maximize their own profit.

When solving a bilevel (leader-follower) game, we usually have an optimization problem embedded as constraints in another optimization problem. In case we can rewrite the inner (lower level) optimization problem in the form of equivalent variational inequalities or KKT conditions, we can transform the bilevel problem into a single level optimization problem that consists of equilibrium constraints (e.g. complementarity conditions). Because of the existence of this constraints, such single level problems are called mathematical programs with equilibrium constraints (MPEC) (Luo et al. 1996). MPECs have been 
extensively studied and used in many different applications and industries including energy, transportation and production. For example, refer to Koh (2012), Allevi et al. (2018), and Siddiqui and Christensen (2016). MPECs are considered as non-linear programming (NPL), and special algorithms have been developed to solve them.

In our biofuel supply chain, refineries are competing for the farmer's land, and each refinery solves a bilevel problem in which the refinery (leader) maximizes his profit at the upper level problem and the farmer (follower) maximizes her profit at the lower level problem. Because of the convexity of the farmer's problem, we can replace it with its KKT conditions and include them in the refineries' problems as new constraints and solve the resultant single level problem (MPEC). Having derived the MPEC for each of the refineries, we need to jointly consider all MPECs to obtain the Generalized Nash Equilibrium, which is one of the main objectives of this chapter. For that, we obtain the KKT conditions of each single level problem and combine them into one single optimization problem. The new problem is called equilibrium problems with equilibrium constraints (EPEC), which has been previously addressed in other works and industries, especially electricity market (e.g. Pozo, \& Contreras 2011; Ruiz et al. 2012 and Kazempour et al. 2013), but not in biofuel supply chain.

As previously discussed, environmental concerns in a BSC have been raised in the literature and in practice; and a government can deal with this issue by providing subsidies to the advanced biofuel industry. Advanced biofuel has several environmental advantages over conventional biofuel (e.g. having higher carbon capture capacity and being less water-intensive), which makes subsidizing its producers feasible. This research contributes to the literature by (1) addressing the competition between existing conventional refineries and emerging advanced biofuel refineries, and exploring the behavior of farmers and refineries, (2) considering subsidies paid to farmers to grow energy crops and second-generation refineries to produce advanced biofuel and studying the effect of that on the supply chain performance, (3) considering the possibility of land expansion or any other diseconomy of scale for the farmers through utilizing increasing marginal cost structure, and finally (4) taking food and fuel market demand function into consideration. In 
particular, a leader-follower game model is designed to capture the behavior of players in equilibrium under the above-mentioned circumstances.

Table 1.1 shows a summary of the most relevant works to ours, grouped based on the discussed features in the literature review along with the features of our work.

Table 1.1 Summary of the most relevant works

\begin{tabular}{|c|c|c|c|c|c|c|c|c|c|c|c|}
\hline \multirow[b]{2}{*}{ Paper } & \multicolumn{2}{|c|}{$\begin{array}{l}\text { Level of } \\
\text { Decision } \\
\text { Making }\end{array}$} & \multicolumn{3}{|c|}{ Policy } & \multicolumn{2}{|c|}{$\begin{array}{l}\text { Supply } \\
\text { Chain } \\
\text { Structure }\end{array}$} & \multicolumn{2}{|c|}{ Method } & \multicolumn{2}{|c|}{ Uncertainty } \\
\hline & 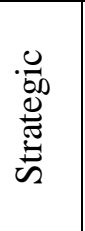 & 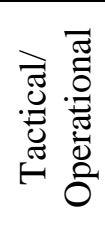 & 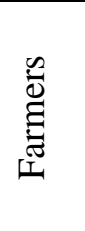 & 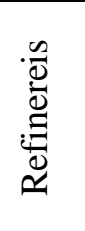 & 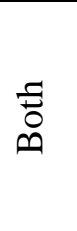 & 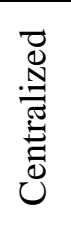 & 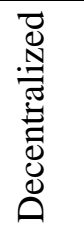 & 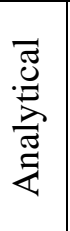 & 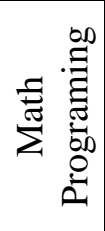 & 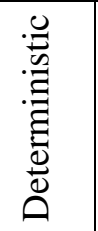 & 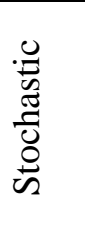 \\
\hline Marufuzzaman et al. (2014) & $\checkmark$ & $\checkmark$ & & $\sqrt{ }$ & & $\sqrt{ }$ & & & $\checkmark$ & & $\checkmark$ \\
\hline Bai et al. (2012) & $\checkmark$ & $\checkmark$ & & & & & $\checkmark$ & & $\checkmark$ & & $\checkmark$ \\
\hline Huang et al. (2014) & $\checkmark$ & $\checkmark$ & & & & & $\checkmark$ & & $\checkmark$ & $\checkmark$ & \\
\hline Yue and You (2014) & $\checkmark$ & $\checkmark$ & & & & & $\checkmark$ & & $\checkmark$ & $\checkmark$ & \\
\hline Bai et al. (2016) & $\checkmark$ & $\checkmark$ & $\checkmark$ & & & & $\sqrt{ }$ & & $\sqrt{ }$ & $\checkmark$ & \\
\hline $\begin{array}{l}\text { Cobugloglu and Buyuk } \\
\qquad(2015)\end{array}$ & & $\checkmark$ & & & & $\checkmark$ & & & $\checkmark$ & $\checkmark$ & \\
\hline Awudu and Zhang (2013) & & $\checkmark$ & & & & $\checkmark$ & & & $\checkmark$ & & $\checkmark$ \\
\hline Nasiri and Zaccour (2009) & & $\checkmark$ & & $\sqrt{ }$ & & & $\checkmark$ & $\checkmark$ & & $\checkmark$ & \\
\hline Luo and Miller (2013) & & $\checkmark$ & & & $\checkmark$ & & $\checkmark$ & & $\checkmark$ & $\checkmark$ & \\
\hline Our work & & $\checkmark$ & & & $\checkmark$ & & $\checkmark$ & $\sqrt{ }$ & $\checkmark$ & $\checkmark$ & \\
\hline
\end{tabular}




\section{Chapter 2 \\ Benchmark equilibrium analysis on the BSC}

\subsection{Introduction}

In this chapter, we consider a region-based biofuel supply chain and analyze it under various cases based on our assumptions regarding the food market and farmer's production cost structure. An extensive problem description along with the considered cases and notations are given in Section 2.2. In Section 2.3 we find the price and quantity equilibrium for the cases and perform a socio-economic analysis. A policy analysis to find the required amount of subsidy and its distribution among the firms is performed in Section 2.4. We conclude the chapter in Section 2.5 with the most remarkable findings from the chapter

\subsection{Problem description}

We consider a biofuel supply chain with three firms: a corn-based refinery, an energy crop-based refinery (named R1 and R2, respectively, hereafter), and a union of the farmers (Figure 2.1), all trying to maximize their own profits. The farmer can grow either her conventional crop (corn), only one type of energy crop or both on her land. Corn can be sold either to a corn market or R1, while energy crop can be sold to R2 only as there is no significant market for this type of crop currently in the U.S.

In the benchmark setting, we assume that, because of the logistics expenses imposed to the farmer or the uncertainty of crop prices at the selling season, the corn market is not a priority for the farmer to sell her crops to, and consequently, refineries are considered as farmer's only customers. In another setting, the farmer sells her corn to a food market as well. The government subsidizes the farmer and R2 to support advanced biofuel production because of its environmental advantages and announces the value of subsidies (dollars per unit quantity) publicly. Knowing the subsidy, refineries announce their quote $\left(p_{1}, p_{2}\right)$ to the farmer for corn and energy crop, respectively, at the beginning of the growing season to secure their production, and then the farmer makes decisions on the use of her land for corn or energy crop $\left(q_{0}, q_{1}, q_{1}\right)$ to 
maximize its profit. We implicitly assume in this research that refineries do not quote lower than the minimum marginal cost of the farmer. We also assume a linear price, or inverse demand function in the form of $\mathrm{P}()=.\mathrm{a}-\mathrm{b}($.$) for both markets (corn and biofuel), to which the farmer and refineries sell their$ products, respectively. The outputs of refineries are homogenous and can be sold in the same biofuel market, where the price is determined by the total realized biofuel production.

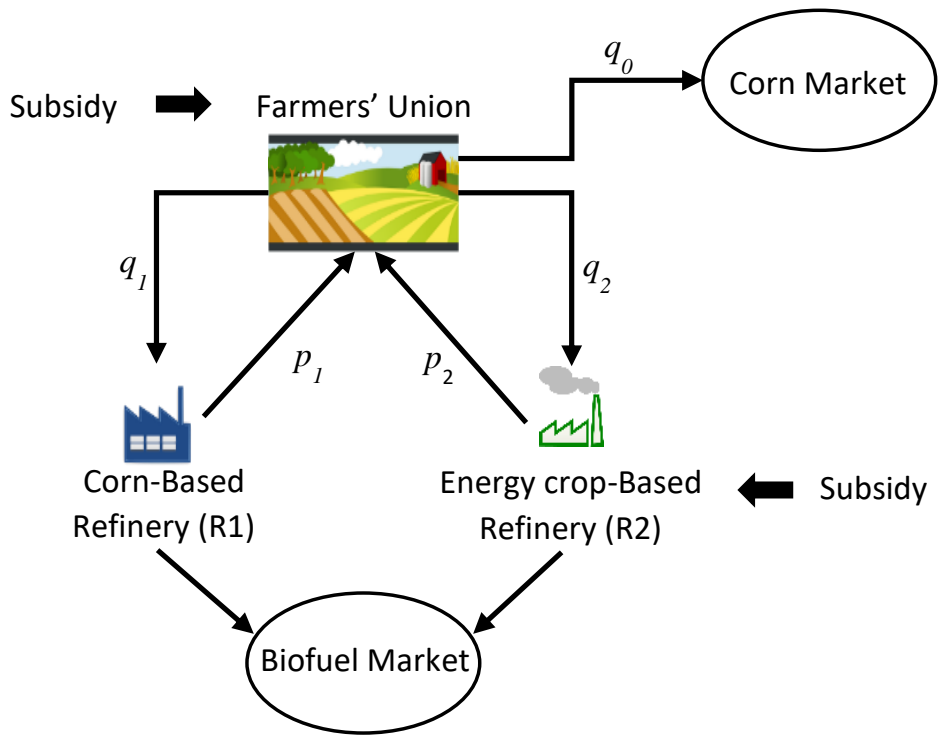

Figure 2.1 Biofuel supply chain with subsidy

This work considers a single farmer. Small farmers in a region can and actually often do form a union, which brings several advantages for them such as better leverage in price negotiation and greater ability entering markets to which they cannot access individually (Agbo et al. 2015). Tchami (2007) mentions that forming a union enables small farms to attain advantage from economies of scale through uniting together and lowering their costs (other examples at Camanzi et al. 2011; Jang and Klein, 2011). In some developed countries, there are large private agricultural corporations who have a high market share and produce a lot of farm products (Cargill ${ }^{1}$, established in 1865, and Monsanto are examples of such companies). In our model, the representative farmer can make land-use decisions in a region.

\footnotetext{
${ }^{1}$ https://www.cargill.com
} 
This work considers two types of farmer's production cost structure. Depending on factors such as geographical or financial conditions, a farmer in a region may have an option to expand her land in various ways such as leasing more farmlands, deforesting the wood lands or even utilizing her own marginal lands on which she did not grow any crops. Depending on whether the farmer can expand her land or not, she faces different types of production cost. Land expansion requires capital investment, and it may not be economically viable beyond some point for small-sized farmers, and consequently, they may face an increasing marginal cost of production. Application of fertilizers for increased production can also cause increasing marginal production cost. At first, the crop yield can be increased with small amount of fertilizer, but, beyond some level, more fertilizer is needed. Some examples of increasing MC are: (a) for daily regular production, we can easily increase the production quantity; however, after some point, it becomes more difficult because we need to use over time. (b) Sometimes, we need to do outsourcing when it is beyond our capacity, which requires higher cost.

It needs to be mentioned that we only consider the increasing part of the total cost curve for the increasing marginal cost function. This type of production cost has been used to capture the diseconomies of scale as the farmland grows. Wickens et al. (1973) argue that farmers may need to finance their farm expansion and advocated a quadratic production cost structure. Peterson (1997) studied the total expenses of corn farms in Midwest and argues that, as the farm size increases, the economy of scale disappears considering other sources of cost such as management, farm dwelling, and off-farm employment (other examples can be found at Parikh (1979), Nasiri and Zaccour (2009), Agbo et al. (2015), Alizamir et al. (2015). In this work, we consider both linear and quadratic production cost structure of the farmer to model both the unavailability and availability of land expansion.

Table 2.1 summarizes the notations in this work, and Table 2.2 summarizes the realistic value of the parameters used in the parametric analyses along with the sources. 
Table 2.1 Notations

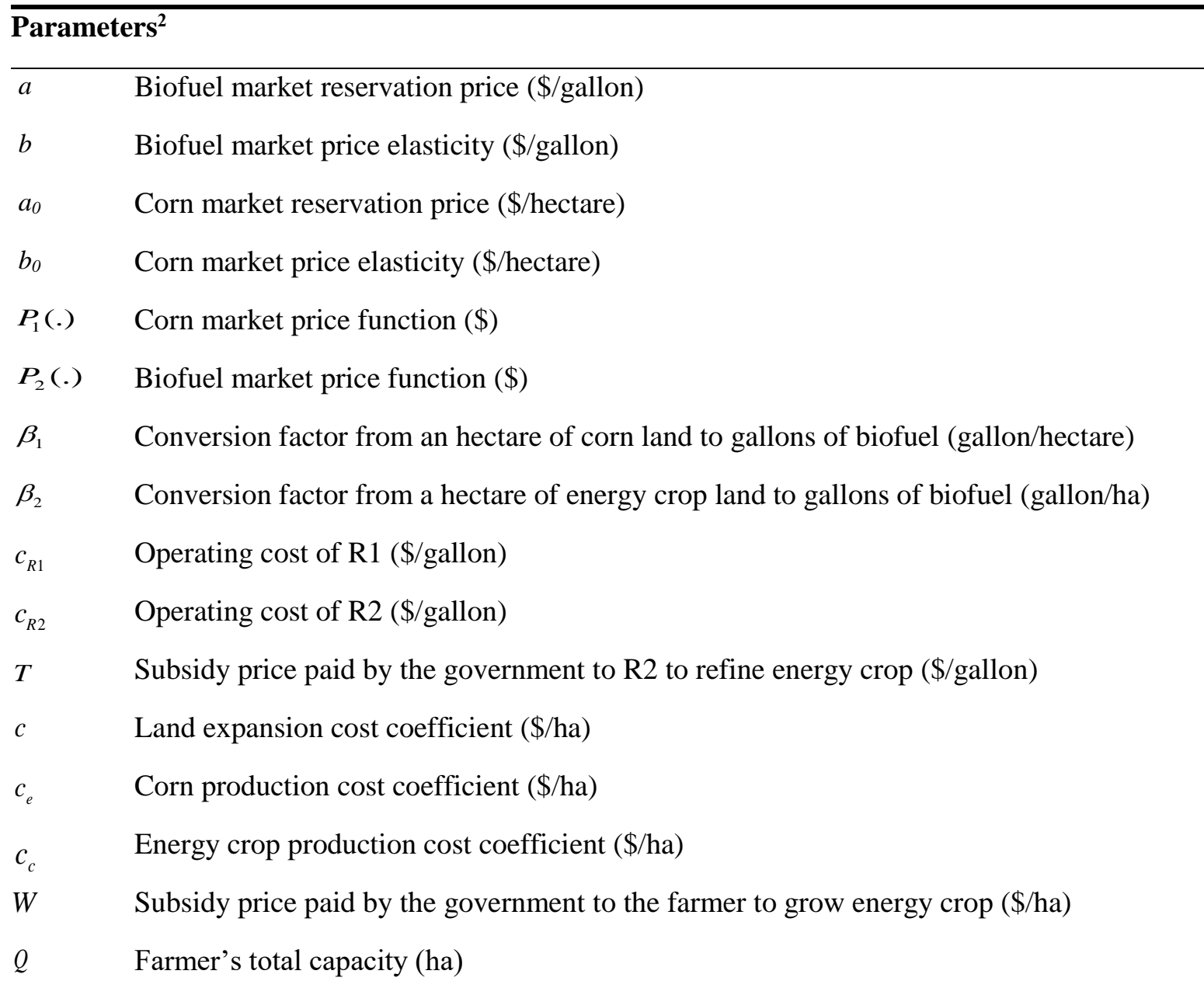

\section{Decision variables}

$q_{0} \quad$ Hectares of land used to grow corn for the local food market (ha)

$q_{1} \quad$ Hectares of land used to grow corn for the refinery R1 (ha)

$q_{2} \quad$ Hectares of land used to grow energy crop for the refinery R2 (ha)

$p_{1} \quad$ Price proposed to the farmer by R1 for a hectare of land allocated to corn $(\$ / \mathrm{ha})$

$p_{2} \quad$ Price proposed to the farmer by R2 for a hectare of land allocated to energy crop ( $\left.\$ / \mathrm{ha}\right)$

\footnotetext{
${ }^{2}$ All parameters are positive values.
} 
Table 2.2 Parameter values used in the parametric analysis

\begin{tabular}{|c|c|c|c|}
\hline $\begin{array}{l}\text { Parameter } \\
\text { value }\end{array}$ & Source & $\begin{array}{l}\text { Parameter } \\
\text { value }\end{array}$ & Source \\
\hline$a=\$ 10$ & Bai et al. (2016) & $c_{R I}=890(\$ / \mathrm{ha})$ & Luo and Miller (2013) \\
\hline$b=0.0005$ & Bai et al. (2016) & $c_{R 2}=1211(\$ / \mathrm{ha})$ & Luo and Miller (2013) \\
\hline$a_{0}=\$ 1500$ & Alizamir et al. (2015) & $c=50(\$ / \mathrm{ha})$ & $\begin{array}{l}\text { Cobuloglu and Buyuktahtakın } \\
\text { (2015) }\end{array}$ \\
\hline$b_{0}=0.0005$ & Alizamir et al. (2015) & $c_{c}=610(\$ / \mathrm{ha})$ & $\begin{array}{l}\text { Cobuloglu and Buyuktahtakın } \\
\text { (2015) }\end{array}$ \\
\hline$\beta_{1}=693(\mathrm{~g} / \mathrm{ha})$ & $\begin{array}{l}\text { Cobuloglu and Buyuktahtakin } \\
\text { (2015) }\end{array}$ & $c_{e}=550(\$ / \mathrm{ha})$ & $\begin{array}{l}\text { Cobuloglu and Buyuktahtakın } \\
\text { (2015) }\end{array}$ \\
\hline$\beta_{2}=890(\mathrm{~g} / \mathrm{ha})$ & $\begin{array}{l}\text { Cobuloglu and Buyuktahtakın } \\
\text { (2015) }\end{array}$ & & \\
\hline
\end{tabular}

\subsection{Equilibrium analysis}

In this section, we present our models for two scenarios: (1) the farmer sells all the crops to refineries only (i.e. there is no food market) (2) the farmer sells the corn to the local food market. Within each scenario, we consider two different production cost structures (constant and increasing marginal cost) of the farmer. With the announced government's decisions of subsidy values, we would have the following sequence of the game where the two refineries move simultaneously under a Bertrand game and then the farmer responds accordingly:

1) R1 and R2 announce their quote for corn and energy crop, respectively, at the same time to the farmer to maximize their profit functions:

$$
\begin{aligned}
& \max _{p_{1}} \pi_{R 1}=P_{2}\left(\beta_{1} q_{1}+\beta_{2} q_{2}\right) \beta_{1} q_{1}-p_{1} q_{1}-c_{R 1} q_{1} \\
& \max _{p_{2}} \pi_{R 2}=P_{2}\left(\beta_{1} q_{1}+\beta_{2} q_{2}\right) \beta_{2} q_{2}-p_{2} q_{2}-c_{R 2} q_{2}+T \beta_{2} q_{2}
\end{aligned}
$$

2) The farmer decides the amount of land for the food market and refineries, $q_{0}, q_{1}$, and $q_{2}$ to maximize her profit:

$$
\max _{q_{1}, q_{2}} \pi_{f}=p_{1} q_{1}+p_{2} q_{2}-g_{1}\left(q_{0}, q_{1}\right)-g_{2}\left(q_{2}\right)+W q_{2}+P_{1}\left(q_{0}\right) q_{0}
$$

Equation (1) is the profit function of R1 consisting of three terms. The first one is the revenue from selling biofuel with the quantity of $\beta_{1} q_{1}$ to the fuel market at the market price of $P_{2}\left(\beta_{1} q_{1}+\beta_{2} q_{2}\right)$, which is a linear 
price function of both refineries' biofuel production quantities. Second and third terms are biostock purchase cost and production cost respectively. Equation (2) is similar to Equation (1), except in the last term that is the subsidy paid to R2 for producing $\beta_{2} q_{2}$ gallons of the energy crop-based biofuel. We have the profit function of the farmer in Equation (3). The first two terms are revenue from selling corn and energy crop to $\mathrm{R} 1$ and $\mathrm{R} 2$ respectively. The next two terms with a negative sign are operating cost of growing corn and energy crop respectively. The fifth term is the subsidy paid to the farmer for allocating $q_{2}$ hectares of land to energy crop and the last term is the revenue from selling corn to the market at the price of $P_{1}\left(q_{0}\right)$.

\subsubsection{Absence of food market}

The first scenario that we consider is the absence of the food market. We previously argued that, for reasons such as high logistics costs to the farmer or the long-term contract that refineries offer, the farmer may be willing to sell the output to the refineries only, which is considered in this subsection under two cases (constant/increasing marginal cost).

\subsubsection{Constant marginal cost (Case 1)}

Using the constant marginal cost structure, we can rewrite the farmer's problem (Equation 3) as follows:

$$
\begin{aligned}
& \max _{q_{1}, \mathrm{q}_{2}} \pi_{F}=\left(p_{1}-c_{c}\right) q_{1}+\left(p_{2}-c_{e}+W\right) q_{2} \\
& q_{1}+q_{2} \leq Q \\
& q_{1}, q_{2} \geq 0
\end{aligned}
$$

The profit function of the refineries would be the same as Equations (1)-(2). The winner (the refinery offering higher marginal profit to the farmer) takes all the farmer's capacity as a result of the Bertrand game. They would share the farmer's land in case of providing equal marginal profits for the farmer. There are three possible cases for which we can find the optimal price of refineries, summarized in Proposition 2.1 .

Proposition 2.1 In the absence of food market and with constant marginal cost structure, the NE are as follows: 
i) if $p_{1}^{\max }-c_{c}>p_{2}^{\max }-c_{e}+W, p_{1}^{*}=p_{2}^{\max }-c_{e}+W+c_{c}+\varepsilon, p_{2}^{*}=p_{2}^{\max }$

$$
q_{1}^{*}=Q, q_{2}^{*}=0
$$

ii) if $p_{1}^{\max }-c_{c}<p_{2}^{\max }-c_{e}+W, p_{2}^{*}=p_{1}^{\max }-c_{c}+c_{e}-W_{c}+\varepsilon, p_{1}^{*}=p_{1}^{\max }$

$$
q_{1}^{*}=0, q_{2}^{*}=Q
$$

iii) if $p_{1}^{\max }-c_{c}=p_{2}^{\max }-c_{e}+W, p_{1}^{*}=p_{1}^{\max }, p_{2}^{*}=p_{2}^{\max }$

$$
q_{1}^{*}=q_{2}^{*}=\frac{Q}{2}
$$

See Appendix A.1.

In Proposition 2.1, $p_{1}^{\max }=a \beta_{1}-b \beta_{1}^{2} Q-c_{R 1}$ and $p_{2}^{\max }=a \beta_{2}-b \beta_{2}^{2} Q-c_{R 2}+T \beta_{2}$ (obtained from the proof) are the highest prices that R1 and R2 can pay while maintaining non-negative profit if they are given all the farmer's land. Intuitively, if there is no subsidy payment, the more efficient refinery would have a higher maximum price and would win the competition by quoting a little bit higher the other one, while the subsidy payment will be in favor of R2 and makes him more competitive against R1. Given the maximum prices of refineries, Proposition 2.1 provides a full comparison of the farmer's marginal profits and the resulting NE. In the first case of Proposition 2.1, marginal profit of growing corn is higher, and the farmer would allocate all the land to R1. The second case is the opposite of the first one where the farmer allocates all the land to $\mathrm{R} 2$, and the third case is the one where refineries share the farmer's land equally. This result illustrates the effect of subsidy plans on the NE such that it makes R2 more competitive against R1 despite possibly being less efficient $\left(c_{R 2} \geq c_{R 1}\right.$ ) and reveals the importance of the government's role in this game. A government can use this result to find the minimum budget required to increase the advanced biofuel production.

Using a parametric analysis with realistic parameter values from the literature, we study the effect of subsidy values on the profit of players (Figure 2.2) as well as on the social welfare of the supply chain (Figure 2.3). From Figure 2.2a, the farmer will be better off under both subsidy plans. From Figure 2.2b-c, it is observed that R2 becomes competitive enough against R1 and makes a positive profit only at higher values of $T(T=2)$. The opposite happens in $T=0,1$. 
Figure 2.3 illustrates the total social welfare (SW) of the supply chain; consisting of (1) the total profit of the firms considered as producer surplus (PS), (2) consumer surplus (CS) at the fuel market, (3) the total subsidy paid by the government under various policies, and sum of (1) and (2) as total social welfare (SW) increase. At lower values of $T, \mathrm{R} 1$ wins the competition and $\mathrm{R} 2$ will not produce anything so no subsidy is paid, and the total SW remains the same. However, at $T=2, \mathrm{R} 2$ price outs R1 by the help of subsidy. With more subsidy on the refinery, the total subsidy paid increases and PS increases. However, the CS of the supply chain declines due to the shrinkage of biofuel production, reflected on SW. The reason behind this reduction lies in the lower efficiency (conversion rate from energy-crop to biofuel) of R2. Fewer biofuel is produced if R2 wins the competition, hence the biofuel price increases, and CS is reduced.

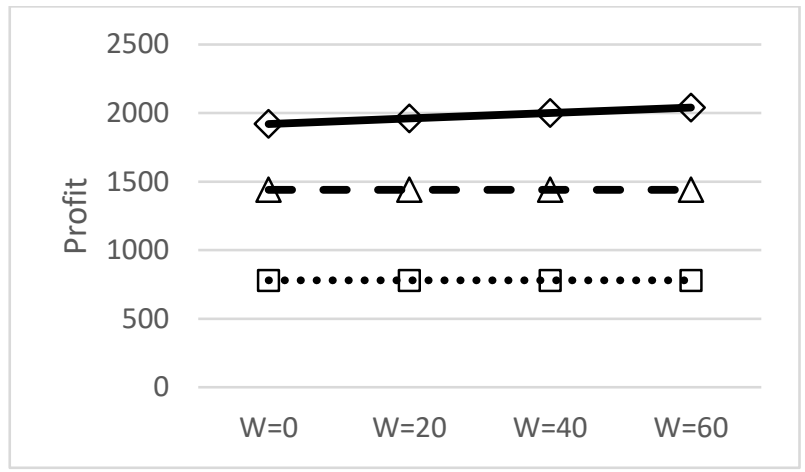

(a) Profit of the farmer

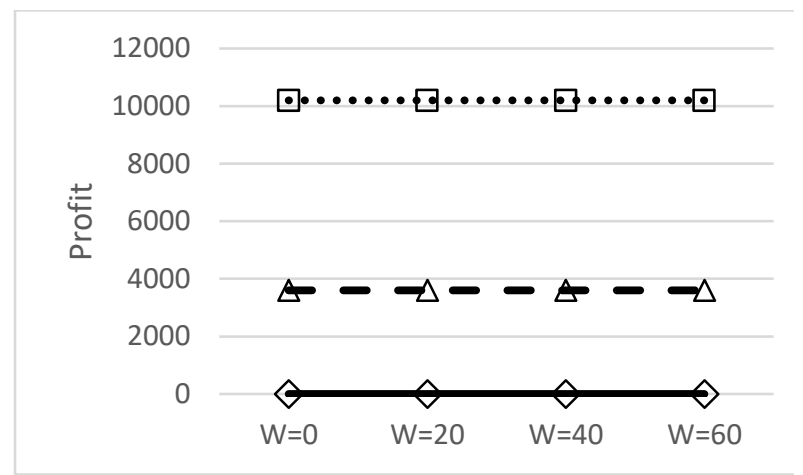

(b) Profit of corn-based refinery (R1)

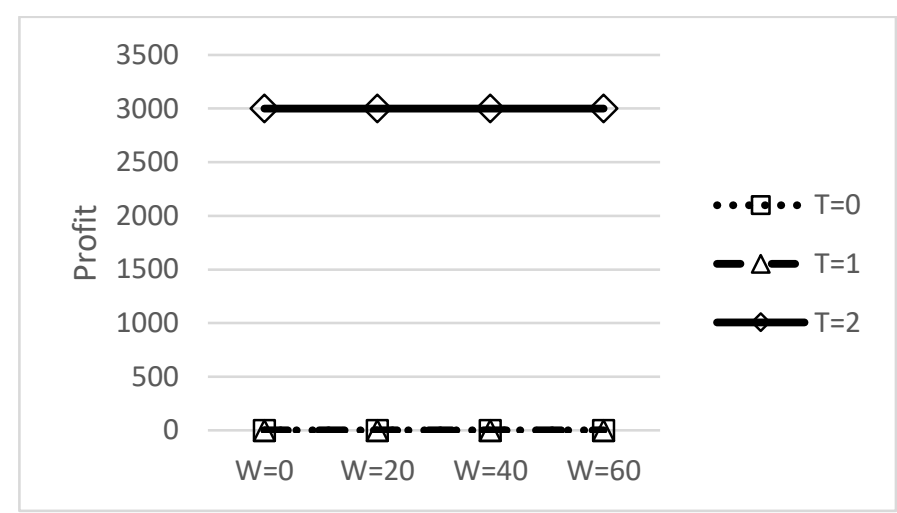

(c) Profit of energy crop-based refinery (R2)

Figure 2.2 Profit of the players under various subsidy policies (Case 1) 


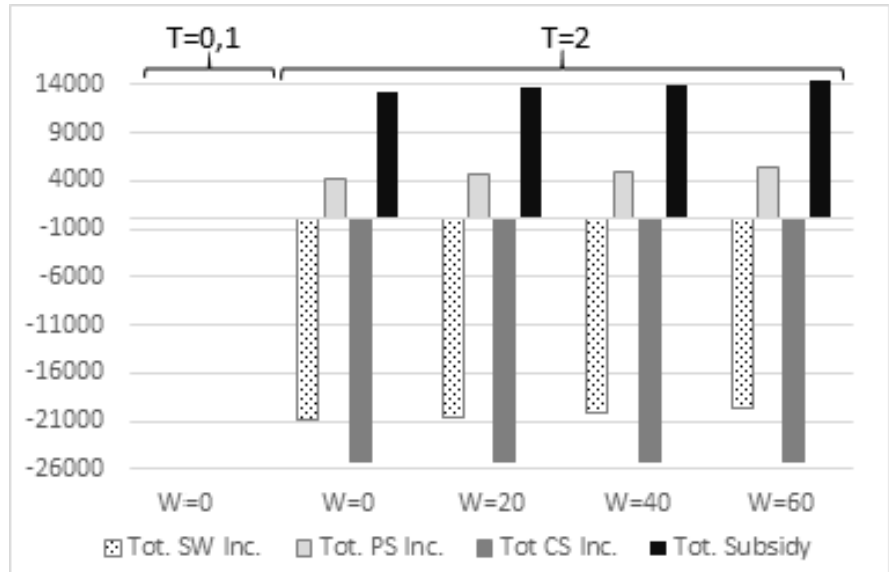

Figure 2.3 Total SW increase breakdown under various policies vs. total subsidy paid (Case 1) Tot. SW Inc. $=$ Total SW increase from the $(W=0, T=0)$ policy

Tot. PS Inc. $=$ Total PS increase from the $(W=0, T=0)$ policy

Tot. CS Inc. $=$ Total CS increase from the $(W=0, T=0)$ policy

\subsubsection{Increasing marginal cost (Case 2)}

With the increasing marginal cost of production from land expansion, we derive the equilibrium of refineries' and the farmer's decisions by backward induction. The farmer's problem can be rewritten as:

$$
\begin{aligned}
& \max _{q_{1}, q_{2}} \pi_{F}=p_{1} q_{1}+p_{2} q_{2}-\left[c q_{1}^{2}+c_{c} q_{1}\right]-\left[c q_{2}^{2}+c_{e} q_{2}\right]+W q_{2} \\
& q_{1}+q_{2} \leq Q \quad\left(\lambda_{3}\right) \\
& q_{1}, q_{2} \geq 0 \quad\left(\lambda_{1}, \lambda_{2}\right)
\end{aligned}
$$

This convex optimization problem can be represented by the Karush-Kuhn-Tucker (KKT) conditions as below and solved for the best responses (BR) of the farmer.

$$
\begin{aligned}
& L=\pi_{f}+\lambda_{3}\left(Q-q_{1}-q_{2}\right)+\lambda_{1} q_{1}+\lambda_{2} q_{2} \\
& \partial L / \partial q_{1}=p_{1}-2 c q_{1}-c_{c}-\lambda_{3}+\lambda_{1}=0 \\
& \partial L / \partial q_{2}=p_{2}-2 c q_{2}-c_{e}+W-\lambda_{3}+\lambda_{2}=0 \\
& 0 \leq \lambda_{1} \perp q_{1} \geq 0
\end{aligned}
$$


$0 \leq \lambda_{2} \perp q_{2} \geq 0$

$0 \leq \lambda_{3} \perp Q-q_{1}-q_{2} \geq 0$

In Equations (4)-(8), $\lambda_{i} \mathrm{~s}$ are KKT multipliers or the shadow price of constraints. Among all possible solutions of Equations (4)-(8), we are more interested in those where the capacity constraint of the farmer is binding since it is only in those solutions that refineries compete on the price. Otherwise (i.e. if the capacity constraint is not binding) there would be no competition between refineries, and the farmer would be able to make land use decision for each refinery without considering the offered price of the other one. The farmer's BR depends on the simultaneously offered prices. Hence, we need to find domains of the offered prices space $\left(p_{1}, p_{2}\right)$ where the BR function of the farmer remains in the same form (domain in the $\left(p_{1}, p_{2}\right)$ space, is an area in which the farmer would have a unique form of land allocation decision). Each domain corresponds to a combination of KKT multipliers as shown in Table 2.3, and the graphical illustration of them is shown in Figure 2.4.

In Figure 2.4, domains are represented based on the value of $p_{1}^{\prime}=p_{1}-c_{c}$ and $p_{2}^{\prime}=p_{2}+W-c_{e}$, which are the marginal profit of the farmer from corn and energy crop evaluated at $q_{1}=0$ and $q_{2}=0$, respectively. In domains $2-4$, the farmer utilizes all her land $\left(\lambda_{3}>0\right)$ while in domain 1 , which has multiple subcases, the land is underutilized $\left(\lambda_{3}=0\right)$, and there is no competition between refineries. In domain 2 (3), the farmer allocates all her capacity to R2 (R1) whereas in domain 4, refineries would share the land (not necessarily equally). In Figure 2.4, $2 c Q$ is derived from the positivity conditions on the farmer's basic variables and the Lagrangian multipliers of its KKT conditions as shown in Equations (6)-(8). In Table 2.3, we show the conditions under which the equilibrium would fall in either of these four domains as well as the corresponding BR of the farmer and the value of KKT multipliers for those domains. 


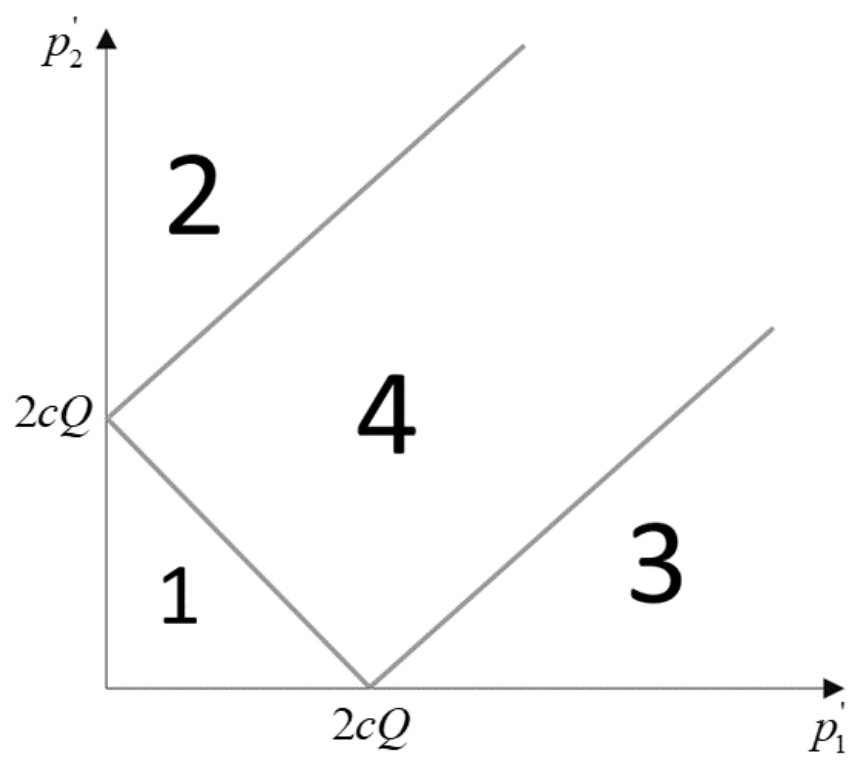

Figure 2.4 Domains of the price offers space $\left(p_{1}^{\prime}, p_{2}^{\prime}\right)$ in Case 2

Table 2.3 Domains, conditions on offered prices and the farmer's BR in Case 2

\begin{tabular}{|c|c|c|c|c|}
\hline Domain & $\left(\lambda_{1}, \lambda_{2}, \lambda_{3}\right)$ & Conditions & Farmer's BR & $\lambda_{i}$ \\
\hline 1 & $(0,0,0)$ & $p_{2}-c_{e}+W+p_{1}-c<2 c Q$ & $\begin{array}{l}q_{1}^{*}=\frac{p_{1}^{*}-c_{c}}{2 c} \\
q_{2}^{*}=\frac{p_{2}^{*}-c_{e}+W}{2 C}\end{array}$ & $\begin{array}{l}\lambda_{1}=0 \\
\lambda_{2}=0 \\
\lambda_{3}=0\end{array}$ \\
\hline 2 & $(+, 0,+)$ & $p_{2}-c_{e}+W-p_{1}+c_{c}>2 c Q$ & $\begin{array}{l}q_{1}^{*}=0 \\
q_{2}^{*}=Q\end{array}$ & $\begin{array}{l}\lambda_{2}=0 \\
\lambda_{1}=\lambda_{3}-p_{1}^{\prime} \\
\lambda_{3}=p_{2}^{\prime}-2 c Q\end{array}$ \\
\hline 3 & $(0,+,+)$ & $p_{1}-c_{c}-p_{2}+c_{e}-W>2 c Q$ & $\begin{array}{l}q_{1}^{*}=Q \\
q_{2}^{*}=0\end{array}$ & $\begin{array}{l}\lambda_{1}=0 \\
\lambda_{2}=\lambda_{3}-p_{2}^{\prime} \\
\lambda_{3}=p_{1}^{\prime}-2 c Q\end{array}$ \\
\hline 4 & $(0,0,+)$ & $\begin{array}{l}p_{2}-c_{e}+W+p_{1}-c \geq 2 c Q \\
p_{2}-c_{e}+W-p_{1}+c \leq 2 c Q \\
p_{1}-c-p_{2}+c_{e}-W \leq 2 c Q\end{array}$ & $\begin{array}{l}q_{1}^{*}=\frac{p_{1}-c_{c}-\lambda_{3}}{2 c} \\
q_{2}^{*}=\frac{p_{2}-c_{e}+W-\lambda_{3}}{2 c}\end{array}$ & $\begin{array}{l}\lambda_{1}=\lambda_{2}=0 \\
\lambda_{3}=\frac{p_{1}^{\prime}+p_{2}^{\prime}-2 c Q}{2}\end{array}$ \\
\hline
\end{tabular}

In the next stage of the game, knowing the farmer's BR, refineries make their decisions accordingly to maximize their own profits. The NE of domains 1-3 are presented in Proposition 2.2 and the NE of domain 4 is shown in Proposition 2.3. 
Proposition 2.2 Under the absence of food market and with a farmer's increasing marginal cost structure, the NE of refineries in domains 1-3 are as follows:

i) In domain $1, p_{1}^{*}=\frac{z e-y f}{x e-y^{2}}, p_{2}^{*}=\frac{z y-x f}{y^{2}-x e}$

ii) In domain $2, p_{2}^{*}=a \beta_{1}-b \beta_{1}^{2} Q-c_{R 1}, p_{1}^{*}<p_{2}^{*}$

iii) In domain $3, p_{1}^{*}=a \beta_{2}-b \beta_{2}^{2} Q-c_{R 2}+T \beta_{2}, p_{2}^{*}<p_{1}^{*}$

The proof and the value of constants (e.g. $z, e, y, f$, and $x$ ) in the NE of domain 1 are shown in Appendix A.2.

Domains 2, 3 can be considered as extreme cases where either of the refineries offers much higher than the other one and is allocated the whole land, while in domain 4, neither of the prices are high enough to win the whole farmer's land. Please note that if $\lambda_{3}=0$ (domain 1), the farmer underutilizes her land, and as was remarked earlier, we can find the optimal value of land allocation for each crop independently from the offered price for the other crop (no competition between refineries).

Proposition 2.3 Under the absence of food market and with the farmer's increasing marginal cost structure, the NE of refineries in domain 4 is as follows:

$$
p_{1}^{*}=\frac{z e-y f}{x e-y d}+c_{c}, p_{2}^{*}=\frac{z d-x f}{y d-x e}-W+c_{e}
$$

The proof and the value of constants are shown in Appendix A.3.

Having the closed form solution of the refineries' optimal decisions, we can explore the impact of subsidy on firms' decisions and their profits. We perform this analysis on the results of domain 4 as shown in Corollary 2.1. Domain 4 is selected because that is the most representative case where all refineries are allocated part of the farmer's land, which makes this domain more interesting. 
Corollary 2.1 If the conversion factor (gallons/ha) of corn is higher than that of energy crop $\left(\beta_{1}>\beta_{2}\right)$, the following holds for the effect of policies on the supply chain's decision variables:
(1) $\frac{\partial p_{1}}{\partial W}, \frac{\partial p_{1}}{\partial T}>0$
(2) $\frac{\partial p_{2}}{\partial W}<0$
(3) $\frac{\partial p_{2}}{\partial T}>0$
(4) $\frac{\partial q_{1}}{\partial W}=-\frac{\partial q_{2}}{\partial W}<0$
(5) $\frac{\partial q_{1}}{\partial T}=-\frac{\partial q_{2}}{\partial T}<0$

Proofs of Corollary 2.1 are provided in Appendix A.4.

Corollary 1 reveals the reaction of refineries and the farmer to the government's subsidies. R1 would announce a higher price for more subsidies (larger $W$ or $T$ ), while the farmer decreases land allocation to R1 despite his higher price offer. Unlike R1, R2 quotes lower price with higher subsidy to the farmer, but the farmer is willing to increase the land allocation to him.

Subsidy policies affect profits and total SW. Figure 2.5 shows the profit of firms at different levels of subsidies $(W, T)$. It is observed that the subsidies bring higher profits for the farmer and R1, but not $\mathrm{R} 2$. Figure 2.6 compares the increase in the total SW (supply chain's profit and biofuel consumer's surplus) and total subsidy paid by the government. It can be observed that though the subsidy increases the total SW, government's expenditure on subsidy is not offset by the gained SW in this case. It is also noticeable that the increase of the SW is due to the increase of PS but not the CS. The reason for the decrease of the CS is the lower efficiency of R2 than R1, which results in the lower biofuel production from the same amount of land.

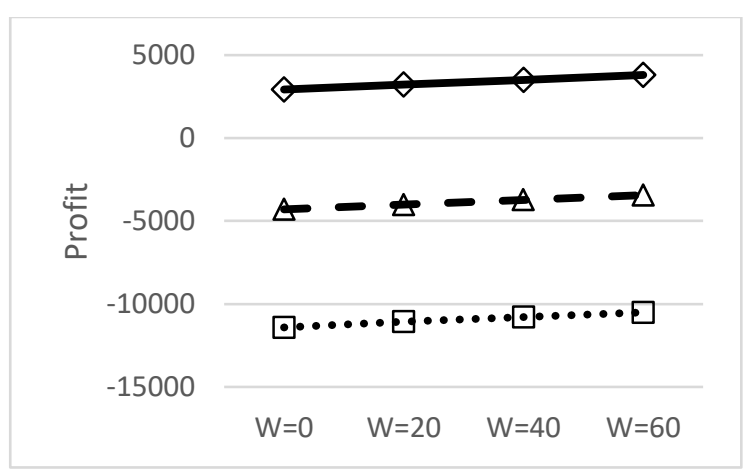

(a) Profit of farmer

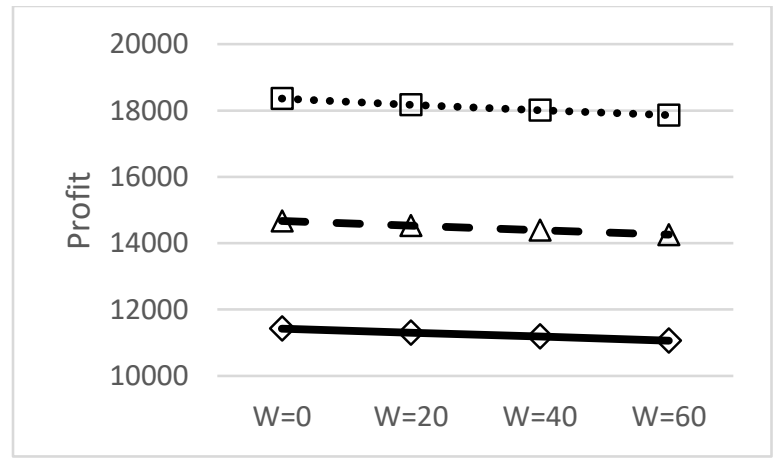

(b) Profit of corn-based refinery (R1) 


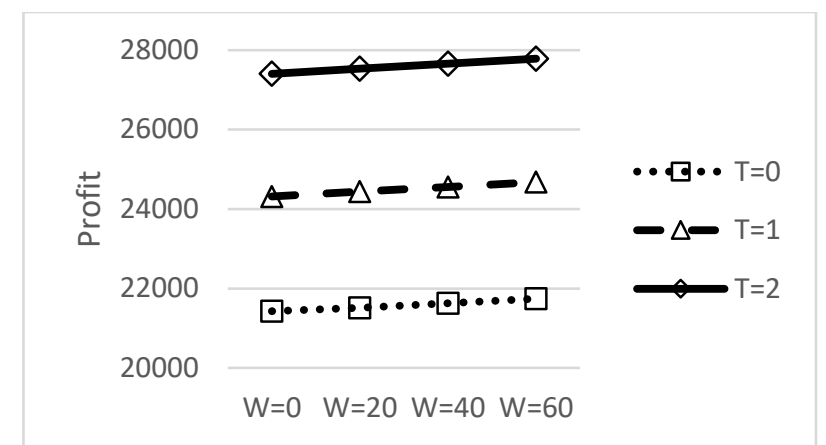

(c) Profit of energy-crop based refinery (R2)

Figure 2.5 Profit of the farmer and refineries under various subsidy policies (Case 2)

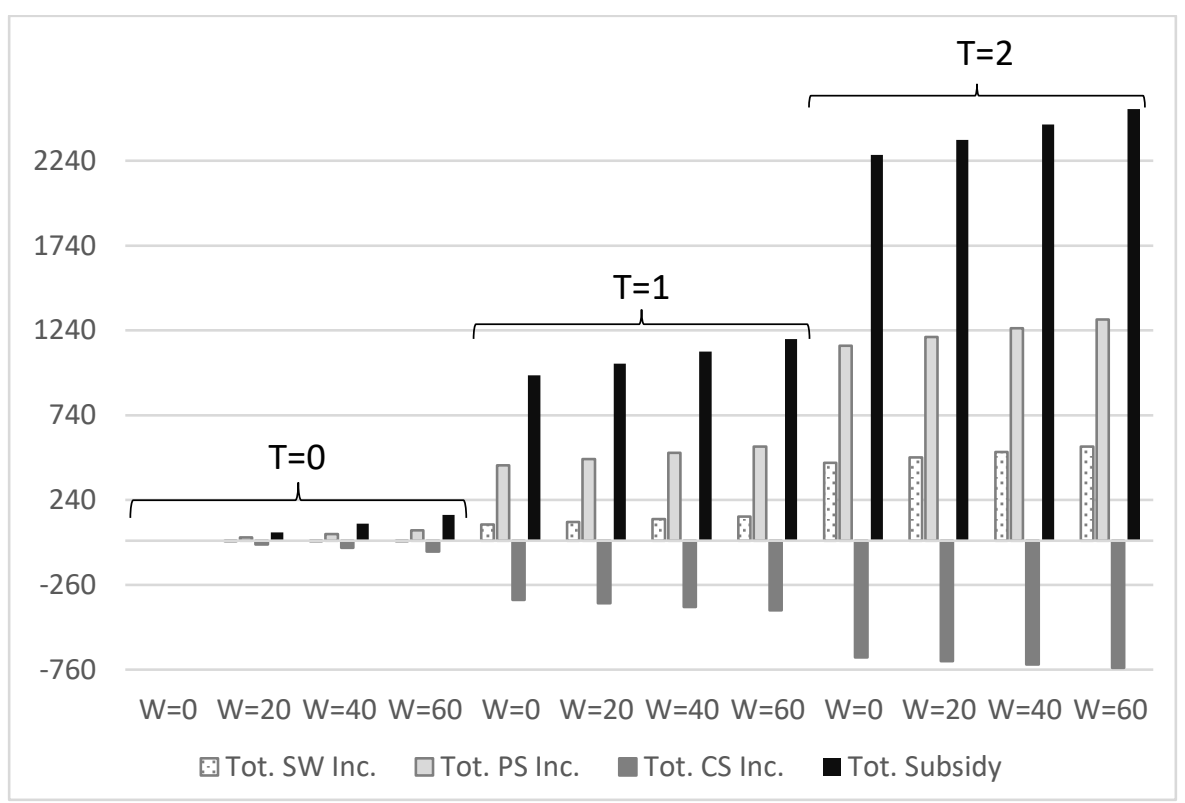

Figure 2.6 Total SW increase breakdown under various policies vs. subsidy paid (Case 2) Tot. SW Inc. $=$ Total $\mathrm{SW}$ increase from the $(\mathrm{W}=0, \mathrm{~T}=0)$ policy

Tot. PS Inc. $=$ Total PS increase from the $(\mathrm{W}=0, \mathrm{~T}=0)$ policy

Tot. $\mathrm{CS}$ Inc. $=$ Total CS increase from the $(\mathrm{W}=0, \mathrm{~T}=0)$ policy

\subsubsection{Presence of food market}

The general form of the farmer's profit function in the presence of food market is as follows:

$\pi_{F}=p_{1} q_{1}+p_{2} q_{2}-g_{1}\left(q_{0}, q_{1}\right)-g_{2}\left(q_{2}\right)+W q_{2}+P_{1}\left(q_{0}\right) q_{0}$ 
In Equation (9), $\mathrm{g}_{1}($.$) and \mathrm{g}_{2}($.$) are representing farmer's cost functions. The last term in Equation (9) is$ the profit of the farmer from allocating $q_{0}$ hectares of land (on which she will grow corn) to the food market with the market price determined by the linear price function $P_{1}($.$) .$

\subsubsection{Constant marginal cost (Case 3)}

The farmer has the following optimization problem:

$$
\begin{array}{ll}
\max _{q_{0}, q_{1}, q_{2}} \pi_{F}=p_{1} q_{1}+p_{2} q_{2}-c_{c}\left(q_{1}+q_{0}\right)-c_{e} q_{2}+W q_{2}+\left(a_{0}-b_{0} q_{0}\right) q_{0} \\
q_{0}+q_{1}+q_{2} \leq Q & \left(\lambda_{3}\right) \\
q_{0}, q_{1}, q_{2} \geq 0 & \left(\lambda_{0}, \lambda_{1}, \lambda_{2}\right)
\end{array}
$$

The KKT conditions of this constrained maximization problem are shown below where $\lambda_{i} \mathrm{~s}$ are the shadow price of constraints.

$$
\begin{aligned}
& L=\pi_{f}+\lambda_{3}\left(Q-q_{0}-q_{1}-q_{2}\right)+\lambda_{0} q_{0}+\lambda_{1} q_{1}+\lambda_{2} q_{2} \\
& \partial L / \partial q_{0}=a_{0}-c_{c}-2 b_{0} q_{0}-\lambda_{3}+\lambda_{0}=0 \\
& \partial L / \partial q_{1}=p_{1}-c_{c}-\lambda_{3}+\lambda_{1}=0 \\
& \partial L / \partial q_{2}=p_{2}-c_{e}+W-\lambda_{3}+\lambda_{2}=0 \\
& 0 \leq \lambda_{0} \perp q_{0} \geq 0 \\
& 0 \leq \lambda_{1} \perp q_{1} \geq 0 \\
& 0 \leq \lambda_{2} \perp q_{2} \geq 0 \\
& 0 \leq \lambda_{3} \perp Q-q_{0}-q_{1}-q_{2} \geq 0
\end{aligned}
$$

If the refineries offer prices at least equal to the marginal cost of the farmer for each crop, the farmer's capacity constraint will be binding under her constant marginal cost. Hence, we focus on the solutions of Equations (10)-(16) in which the farmer utilizes all her land $\left(\lambda_{3} \neq 0\right)$. Similar to the previous case, we need to find all the domains of offered prices under which the farmer would have a corresponding BR. Figure 
2.7 shows all considered domains based on $\left(p_{1}^{\prime}, p_{2}^{\prime}\right)$. Table 2.4 summarizes the conditions on the offered prices for each domain, the corresponding farmer's BR and KKT multipliers.

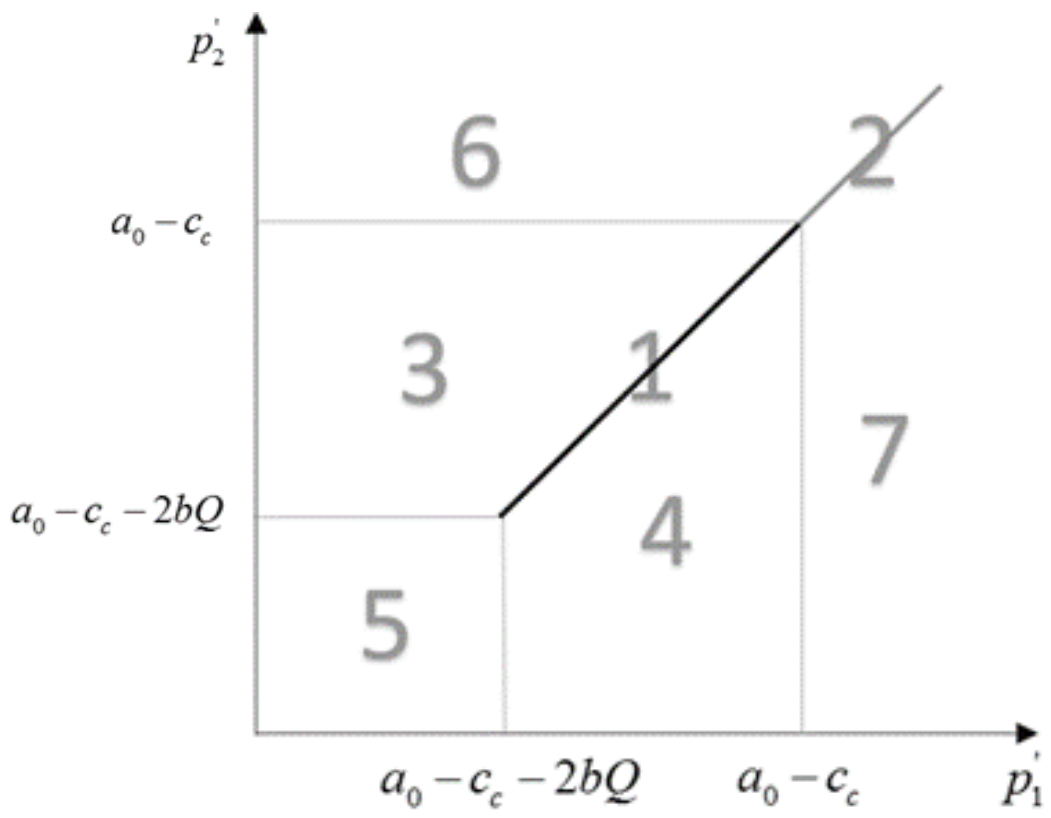

Figure 2.7 Domains of the price offers' space $\left(p_{1}^{\prime}, p_{2}^{\prime}\right)$ in Case 3

In domain 1 , all decision variables $\left(q_{0}, q_{1}, q_{2}\right)$ take positive values meaning that the farmer is willing to allocate her land to all end users including the food market. In this domain, refineries offer prices such that the profit per unit land area from each crop are equal. In domain 2, both refineries offer higher prices than in domain 1 such that the farmer won't sell to the food market and will share its land between only refineries. In domain 3 (4), the farmer will sell to the refinery R2 (R1) and the food market. In the domain 6 (7), the farmer will only sell to the refinery R2 (R1). Finally, in domain 5, the farmer will only sell to the food market.

Table 2.4 Domains, conditions on offered prices and the farmer's BR in Case 3

\begin{tabular}{|c|c|c|c|c|}
\hline Domain & $\left(\lambda_{0}, \lambda_{1}, \lambda_{2}, \lambda_{3}\right)$ & Conditions & Farmer's BR & $\lambda_{i}$ \\
\hline 1 & $(0,0,0,+)$ & $\begin{array}{l}p_{1} \leq a_{0} \\
p_{2}-c_{e}+W \leq a_{0}-c_{c} \\
p_{2}-c_{e}+W=p_{1}-c=\lambda_{3} \\
p_{2}-c_{e}+W \geq a_{0}-c_{c}-2 b_{0} Q \\
p_{1} \geq a_{0}-2 b_{0} Q\end{array}$ & $\begin{array}{l}q_{0}=\frac{a_{0}-c_{c}-\lambda_{3}}{2 b_{0}} \\
q_{1}=q_{2}=\frac{\left(Q-q_{0}\right)}{2}\end{array}$ & $\begin{array}{l}\lambda_{0}=\lambda_{1}=\lambda_{2}=0 \\
\lambda_{3}=p_{1}^{\prime}=p_{2}^{\prime}\end{array}$ \\
\hline
\end{tabular}




$$
\begin{aligned}
& \begin{array}{llll} 
& p_{1}>a_{0} & q_{0}=0 & \lambda_{1}=\lambda_{2}=0 \\
& p_{2}-c_{e}+W>a_{0}-c_{c} & q_{1}=q_{2}=\frac{Q}{2} & \lambda_{0}=\lambda_{3}-a_{0}+c_{c} \\
& p_{2}-c_{e}+W=p_{1}-c_{c} & & \lambda_{3}=p_{1}^{\prime}=p_{2}^{\prime}
\end{array} \\
& 3 \\
& p_{2}-c_{e}+W=\lambda_{3} \\
& p_{2}-c_{e}+W \leq a_{0}-c_{c} \\
& q_{0}=\frac{a_{0}-c_{c}-\lambda_{3}}{2 b_{0}} \quad \lambda_{0}=\lambda_{2}=0 \\
& \begin{array}{lll}
p_{2}-c_{e}+W>p_{1}-c_{c} & q_{1}=0 & \lambda_{1}=\lambda_{3}-p_{1}
\end{array} \\
& p_{2}-c_{e}+W \geq a_{0}-c_{c}-2 b_{0} Q \\
& q_{2}=Q-q_{0} \\
& \begin{array}{l}
p_{1} \leq a_{0} \\
p_{1}>a_{0}-2 b_{0} Q
\end{array} \quad q_{0}=\frac{a_{0}-c_{c}-\lambda_{3}}{2 b_{0}} \quad \lambda_{0}=\lambda_{1}=0 \\
& 4 \\
& (0,0,+,+) \quad p_{1}-c_{c}=\lambda_{3} \\
& p_{2}-c_{e}+W<p_{1}-c_{c} \\
& q_{1}=Q-q_{0} \\
& \lambda_{2}=\lambda_{3}-p_{2}^{\prime} \\
& \lambda_{3}=p_{1}^{\prime} \\
& 5 \\
& (0,+,+,+) \\
& p_{2}-c_{e}+W<a_{0}-c_{c}-2 b_{0} Q \quad q_{0}=Q \\
& p_{1}<a_{0}-2 b_{0} Q \\
& q_{1}=q_{2}=0 \\
& \lambda_{0}=0 \\
& \lambda_{1}=\lambda_{3}-p_{1}^{\prime} \\
& \lambda_{2}=\lambda_{3}-p_{2}^{\prime} \\
& \lambda_{3}=a_{0}-c_{c}-2 b_{0} Q \\
& \lambda_{0}=\lambda_{3}+c_{c}-a_{0} \\
& p_{2}-c_{e}+W>a_{0}-c_{c} \quad q_{0}=q_{1}=0 \quad \lambda_{1}=\lambda_{3}-p_{1}^{\prime} \\
& 6 \\
& (+,+, 0,+) \quad p_{2}-c_{e}+W>p_{1}-c_{c} \\
& q_{2}=Q \\
& \lambda_{2}=0 \\
& \lambda_{3}=p_{2}^{\prime} \\
& \lambda_{0}=\lambda_{3}+c_{c}-a_{0} \\
& 7 \quad(+, 0,+,+) \quad \begin{array}{l}
p_{1}>a_{0} \\
p_{2}-c_{e}+W<p_{1}-c_{c}
\end{array} \\
& \begin{array}{l}
q_{0}=q_{2}=0 \\
q_{1}=Q
\end{array} \\
& \lambda_{1}=0 \\
& \lambda_{2}=\lambda_{3}-p_{2}^{\prime} \\
& \lambda_{3}=p_{1}^{\prime}
\end{aligned}
$$

Equations (10)-(16) are not sufficient to give the closed form solution of all the BR of the farmer for the domains on the lines, but by assuming that the farmer is indifferent between the refineries, equal sharing of the land $\left(q_{1}=q_{2}\right)$ would be a possible solution as given in the Farmer's BR column for domains 1 and 2.

For the follower's decision, the BR of the farmer, we can find the prices of refineries for each domain following the backward induction procedure. Proposition 2.4 summarizes the NE of all domains in this case.

Proposition 2.4 In the presence of food market and with the farmer's constant marginal cost structure, the NE of refineries in domains 1-7 are as follows:

i) In domain $1, p_{1}^{*}=\frac{2 a \beta_{1} b_{0}-2 b b_{0} \beta_{1}^{2} Q+b \beta_{1}^{2} a_{0}-2 b b_{0} \beta_{1} \beta_{2} Q+b \beta_{1} \beta_{2} a_{0}+2 b_{0}\left(a_{0}-c_{R 1}-Q\right)}{4 b_{0}+b \beta_{1} \beta_{2}+b \beta_{1}^{2}}, p_{2}^{*}=p_{1}^{*}-c_{c}+c_{e}-W$ 
ii) In domain $2, p_{1}^{*}=a \beta_{1}-b \beta_{1}^{2} \frac{Q}{2}-b \beta_{1} \beta_{2} \frac{Q}{2}-c_{R 1}, p_{2}^{*}=a \beta_{2}-b \beta_{2}^{2} \frac{Q}{2}-b \beta_{1} \beta_{2} \frac{Q}{2}-c_{R 2}+T \beta_{2}$

iii) In domain $3, p_{2}^{*}=\frac{a \beta_{2} b_{0}-2 b b_{0} \beta_{2}^{2} Q+b \beta_{2}^{2} a_{0}-b \beta_{2}^{2} c_{c}+b \beta_{2}^{2} c_{e}-b \beta_{2}^{2} W-2 b_{0}^{2} Q+b_{0}\left(a_{0}-c_{c}+c_{e}-W-c_{R 2}+T \beta_{2}\right)}{b \beta_{2}^{2}+2 b_{0}}, p_{1}^{*}<p_{2}^{*}$

iv) In domain $4, p_{1}^{*}=\frac{a \beta_{1} b_{0}-2 b b_{0} \beta_{1}^{2} Q+b \beta_{1}^{2} a_{0}-2 b_{0}^{2} Q+a_{0} b_{0}-b_{0} c_{R 1}}{b \beta_{1}^{2}+2 b_{0}}, p_{2}^{*}<p_{1}^{*}$

v) In domain $5, p_{1}^{*}<a_{0}-2 b Q, p_{2}^{*}<a_{0}-c_{c}+c_{e}-W-2 b Q$

vi) In domain $6, p_{2}^{*}=a \beta_{2}-b \beta_{2}^{2} Q-c_{R 2}+T \beta_{2}, p_{1}^{*}<p_{2}^{*}$

vii) In domain $7, p_{1}^{*}=a \beta_{1}-b \beta_{1}^{2} Q-c_{R 1}, p_{2}^{*}<p_{1}^{*}$

The NE shown in Proposition 2.4 can be derived from the first order condition (FOC) of refineries' profit functions. Among the domains, domain 1 is the domain, in which refineries will share the land equally and the food market also receives a positive share from the land. This is the most representative case, and we use the results of this domain to study the effect of subsidies on profits and the total SW increase. The profit of firms under various subsidy plans have been illustrated in Figure 2.8. It is observed that despite the existence of food market, in this case, the farmer is worse off than Case 1 (no food market-linear cost structure) and subsidy plans have no effect on the profit of the farmer. The intuition behind this result is the domain that we are using for this parametric analysis. Domain 1 considers equal land allocation between the refineries and the change of subsidies does not change the resultant equilibrium. The rate of change of R1's profit is positive by the increase of $W$ unlike the previous models (without food market), while $T$ has no effect on that, and the profit of R2 is generally lower in this model than the previous ones (without food market). This result can also be explained by the domain which we have used for the analyses. The most interesting result of this model can be observed in Figure 2.9 where the total SW of the supply chain is compared against the total subsidy paid by the government. It is shown that the government's expenditure (Tot. Subsidy) is completely offset by the increase of the total SW (Tot. SW Inc.) under several policy plans. 
This result shows how the interference of the government in biofuel industry can benefit all the engaged players and consumers.

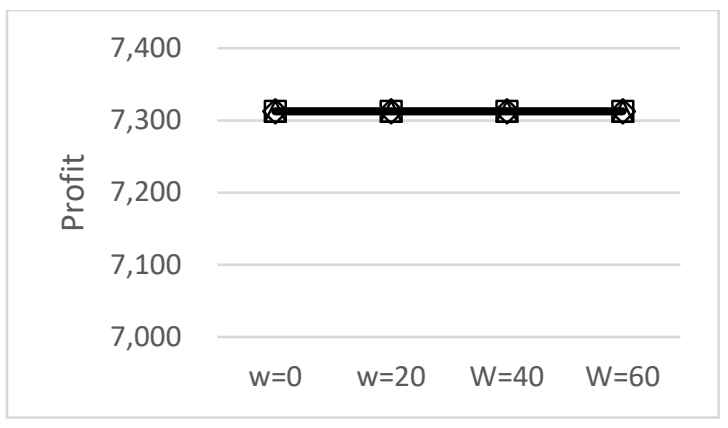

(a) Profit of the farmer

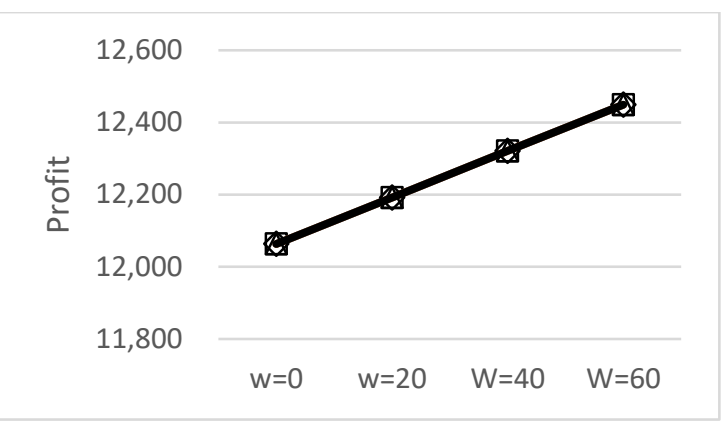

(b) Profit of corn-based refinery (R1)

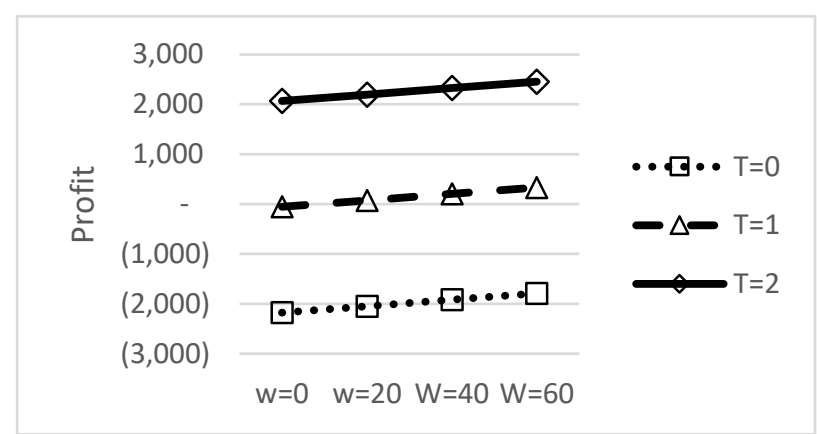

(c) Profit of energy crop-based refinery (R2)

Figure 2.8 Profit of the farmer and refineries under various subsidy policies (Case 3)

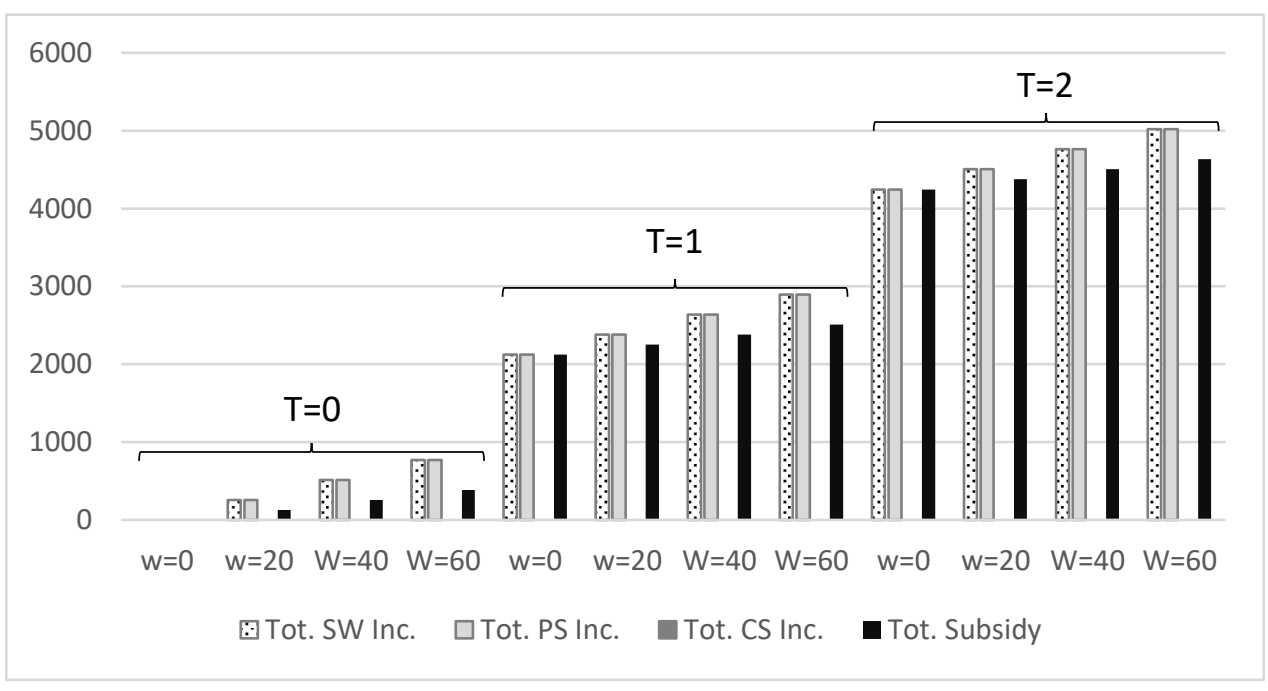

Figure 2.9 Total SW increase breakdown under various policies vs. subsidy paid (Case 3) Tot. SW Inc. $=$ Total SW increase from the $(\mathrm{W}=0, \mathrm{~T}=0)$ policy

Tot. PS Inc. $=$ Total PS increase from the $(\mathrm{W}=0, \mathrm{~T}=0)$ policy

Tot. CS Inc. $=$ Total CS increase from the $(\mathrm{W}=0, \mathrm{~T}=0)$ policy 


\subsubsection{Increasing marginal cost (Case 4)}

In this case, the farmer has the following constrained maximization problem:

$$
\begin{array}{ll}
\max _{q_{0}, q_{1}, q_{2}} \pi_{F}=p_{1} q_{1}+p_{2} q_{2}-\left[c\left(q_{0}^{2}+q_{1}^{2}\right)+c_{c}\left(q_{0}+q_{1}\right)\right]-\left[c q_{2}^{2}+c_{e} q_{2}\right]+W q_{2}+\left(a_{0}-b_{0} q_{0}\right) q_{0} \\
q_{0}+q_{1}+q_{2} \leq Q & \left(\lambda_{3}\right) \\
q_{0}, q_{1}, q_{2} \geq 0 & \left(\lambda_{0}, \lambda_{1}, \lambda_{2}\right)
\end{array}
$$

We derive the KKT conditions of this optimization problem to find the BR of the farmer under various domains of the offered prices. Similar to the previous models, considering the assumption of having positive marginal profits for the farmer, the capacity constraint of the farmer will be binding in all considered solutions. Seven domains were found for this case (case 4), shown in Figure 2.10. The results are summarized in Table 2.5 where $\left(p_{1}^{\prime}, p_{2}^{\prime}\right)=\left(p_{1}-c_{c}, p_{2}-c_{e}+W\right)>0, k=\left(a_{0}-c_{c}\right) c-2 c l Q$, and $l=b_{0}+c$.

$L=\pi_{F}+\lambda_{3}\left(Q-q_{0}-q_{1}-q_{2}\right)+\lambda_{0} q_{0}+\lambda_{1} q_{1}+\lambda_{2} q_{2}$

$\partial L / \partial q_{0}=a_{0}-c_{c}-2 c q_{0}-2 b_{0} q_{0}-\lambda_{3}+\lambda_{0}=0$

$\partial L / \partial q_{1}=p_{1}-2 c q_{1}-c_{c}-\lambda_{3}+\lambda_{1}=0$

$\partial L / \partial q_{2}=p_{2}-2 c q_{2}-c_{e}+W-\lambda_{3}+\lambda_{2}=0$

$0 \leq \lambda_{0} \perp q_{0} \geq 0$

$0 \leq \lambda_{1} \perp q_{1} \geq 0$

$0 \leq \lambda_{2} \perp q_{2} \geq 0$

$0 \leq \lambda_{3} \perp Q_{2}-q_{0}-q_{1}-q_{2} \geq 0$ 
In Figure 2.10, domain 1 is the region where all the farmer's decision variables take positive values. In domain 2, refineries offer higher prices such that the farmer will allocate the whole land to both and nothing to the food market. In domain 3 (4), the farmer will share the land between R2 (R1) and the food market. In domain 5 (6) all the farmer's capacity will be allocated to R2 (R1), and finally, in domain 7, the food market receives all the farmer's capacity. Table 2.5 summarizes the conditions on the offered prices for each domain, the corresponding farmer's BR and KKT multipliers.

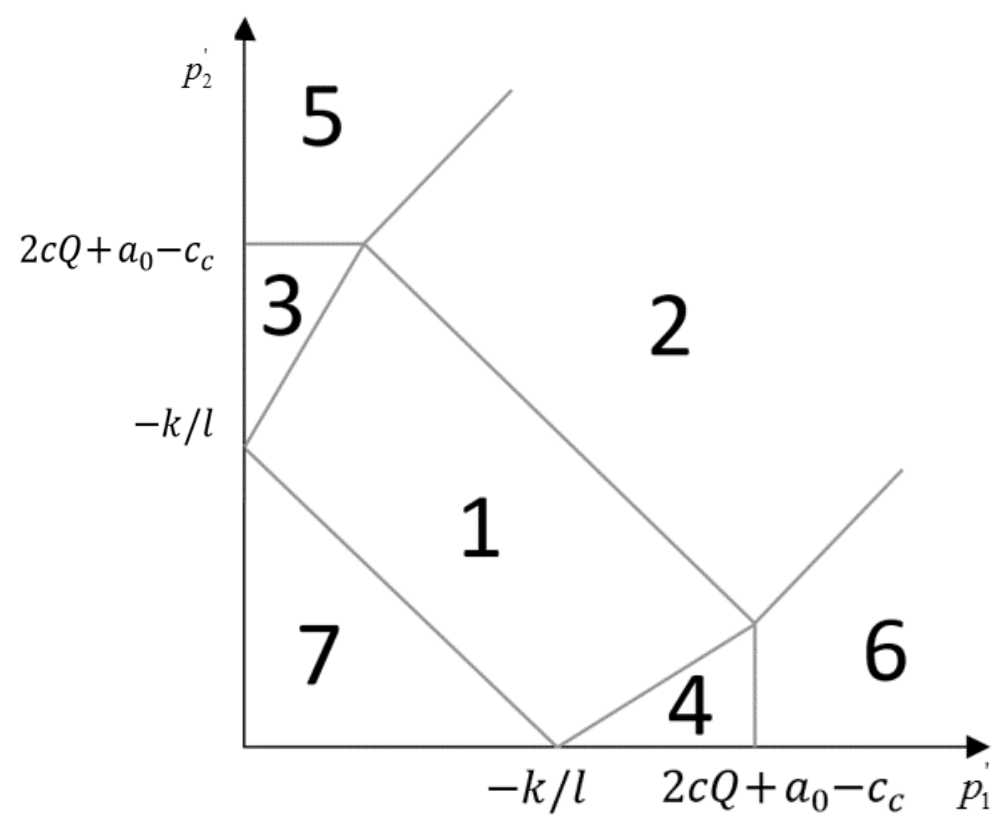

Figure 2.10 Domains of the price offers space $\left(p_{1}^{\prime}, p_{2}^{\prime}\right)$ in Case 4

Table 2.5 Domains, conditions on offered prices and the farmer's BR in Case4

\begin{tabular}{|c|c|c|c|c|}
\hline Domain & $\left(\lambda_{0}, \lambda_{1}, \lambda_{2}, \lambda_{3}\right)$ & Conditions & Farmer's BR & $\lambda_{i}$ \\
\hline 1 & $(0,0,0,+)$ & $\begin{array}{l}p_{1}-c+p_{2}-c_{e}+W>-k / l \\
(l+c)\left(p_{2}-c_{e}+W\right)-l\left(p_{1}-c\right) \geq k \\
(l+c)\left(p_{1}-c\right)-l\left(p_{2}-c_{e}+W\right) \geq k \\
p_{1}-c+p_{2}-c_{e}+W \leq\left((c+2 l)\left(a_{0}-c_{c}\right)-k\right) / l\end{array}$ & $\begin{array}{l}q_{0}=\frac{a_{0}-c_{c}-\lambda_{3}}{2 l} \\
q_{1}=\frac{p_{1}-c_{c}-\lambda_{3}}{2 c} \\
q_{2}=\frac{p_{2}-c_{e}+W-\lambda_{3}}{2 c}\end{array}$ & $\begin{array}{l}\lambda_{0}=\lambda_{1}=\lambda_{2}=0 \\
\lambda_{3}=\frac{k+p_{1}^{\prime} l+p_{2}^{\prime} l}{c+2 l}\end{array}$ \\
\hline 2 & $(+, 0,0,+)$ & $\begin{array}{l}p_{1}-c_{c}+p_{2}-c_{e}+W>2\left(c Q-c_{c}+a_{0}\right) \\
p_{1}-c_{c}-p_{2}+c_{e}-W \geq-2 C Q \\
p_{2}-c_{e}+W-p_{1}+c_{c} \geq-2 C Q\end{array}$ & $\begin{array}{l}q_{0}=0 \\
q_{1}=\frac{p_{1}-c_{c}-\lambda_{3}}{2 c} \\
q_{2}=\frac{p_{2}-c_{e}+W-\lambda_{3}}{2 c}\end{array}$ & $\begin{array}{l}\lambda_{0}=\lambda_{3}+c_{c}-a_{0} \\
\lambda_{1}=\lambda_{2}=0 \\
\lambda_{3}=\frac{p_{1}^{\prime}+p_{2}^{\prime}-2 c Q}{2}\end{array}$ \\
\hline
\end{tabular}




$$
\begin{aligned}
& 3 \quad(0,+, 0,+) \quad \begin{array}{l}
\left(p_{1}-c_{c}\right)(c+l)-\left(p_{2}-c_{e}+W\right) l<k \\
\\
p_{2}-c_{e}+W \leq a_{0}-c_{c}+2 c Q
\end{array} \\
& 4 \quad(0,0,+,+) \quad \begin{array}{l}
\left(p_{1}-c_{c}\right) l-\left(p_{2}-c_{e}+W\right)(c+l)>-k \\
\\
p_{1}-c_{c} \leq a_{0}-c_{c}+2 c Q
\end{array} \\
& \begin{array}{ll}
q_{0}=\frac{a_{0}-c_{c}-\lambda_{3}}{2 l} & \lambda_{0}=\lambda_{2}=0 \\
q_{1}=0 & \lambda_{1}=\lambda_{3}-p_{1}^{\prime} \\
q_{2}=\frac{p_{2}-c_{e}+W-\lambda_{3}}{2 c} & \lambda_{3}=\frac{k+p_{2}^{\prime} l}{c+l}
\end{array} \\
& q_{0}=\frac{a_{0}-c_{c}-\lambda_{3}}{2 l} \quad \lambda_{0}=\lambda_{1}=0 \\
& q_{1}=\frac{p_{1}-c_{c}-\lambda_{3}}{2 c} \quad \lambda_{2}=\lambda_{3}-p_{2}^{\prime} \\
& q_{2}=0 \quad \lambda_{3}=\frac{k+p_{1} l}{c+l} \\
& \begin{array}{llll} 
& & & \\
& p_{2}-c_{e}+W>2 c Q+a_{0}-c_{c} & q_{0}=\lambda_{3}-a_{0}+c_{c} \\
& p_{2}-c_{e}+W-p_{1}+c_{c}>0 & q_{2}=Q & \lambda_{1}=\lambda_{3}-p_{1}^{\prime} \\
& & & \lambda_{2}=0 \\
& & \lambda_{3}=p_{2}^{\prime}-2 c Q
\end{array} \\
& 6 \quad(+, 0,+,+) \quad \begin{array}{l}
p_{1}-c_{c}>2 c Q+a_{0}-c_{c} \\
p_{1}-c_{c}-p_{2}+c_{e}-W>2 c Q
\end{array} \\
& 7 \\
& (0,0,0,0,0,0) \quad p_{1}^{\prime}+p_{2}^{\prime} \leq 2 c Q-\frac{2 c\left(a_{0}-c_{c}\right)}{2 b_{0}+2 c} \\
& \begin{array}{ll}
q_{0}=q_{2}=0 & \lambda_{0}=\lambda_{3}-a_{0}+c \\
q_{1}=Q & \lambda_{1}=0 \\
& \lambda_{2}=\lambda_{3}-p_{2}^{\prime} \\
& \lambda_{3}=p_{1}^{\prime}-2 c Q
\end{array} \\
& q_{0}^{*}=\frac{a_{0}-c_{c}}{2 l} \\
& q_{1}^{*}=\frac{p_{1}^{\prime}}{2 c} \quad \lambda_{i}=0 \\
& q_{2}^{*}=\frac{p_{2}^{\prime}}{2 c}
\end{aligned}
$$

For the leader's decision (refineries decision), having the BR of the farmer and following the backward induction procedure, the closed form solution of the NE of the prices is derived for each of the domains. In the following, we present the NE of domain 1 in Proposition 2.5, the NE of domain 2 in Proposition 2.6 and the NE of other domains in Proposition 2.7.

Proposition 2.5 In the presence of food market and with a farmer's increasing marginal cost structure, the NE of refineries in domain 1 are as follows:

$$
p_{1}^{*}=\frac{z e-y f}{x e-y d}+c_{c}, p_{2}^{*}=\frac{z d-x f}{y d-x e}-W+c_{e}
$$

where, 


$$
\begin{aligned}
& x=\frac{1}{2 c}\left(-k_{2}+k_{2} k_{7}+k_{3} k_{7}-k_{4}+k_{4} k_{7}-1+k_{7}\right)-k_{5}, \\
& y=\frac{1}{2 c}\left(k_{2} k_{7}-k_{3}+k_{3} k_{7}+k_{4} k_{7}+k_{7}\right), \\
& z=-k_{1}-\frac{k_{6}}{2 c}\left(k_{2}+k_{3}+k_{4}+1\right), \\
& d=\frac{1}{2 c}\left(r_{2} k_{7}-k_{3}+k_{3} k_{7}+r_{4} k_{7}+k_{7}\right), \\
& e=\frac{1}{2 c}\left(-r_{2}+r_{2} k_{7}+k_{3} k_{7}-r_{4}+r_{4} k_{7}-1+k_{7}\right)-k_{5}, \\
& f=-r_{1}-\frac{k_{6}}{2 c}\left(r_{2}+k_{3}+r_{4}+1\right) .
\end{aligned}
$$

The value of $k_{i}^{\prime} \mathrm{s}, i=1, \ldots, 7$ and $r_{i}^{\prime} s, i=1, \ldots, 4$ are summarized in Appendix A.5. The NE is found by simultaneously solving the FOC of the refineries' profit function given the BR of the farmer in domain 1.

Proposition 2.6 In the presence of food market and with the farmer's increasing marginal cost structure, the NE of refineries in domain 2 are as follows:

$$
p_{1}^{*}=\frac{z e-y f}{x e-y d}+c_{c}, p_{2}^{*}=\frac{z d-x f}{y d-x e}-W+c_{e}
$$

where,

$$
\begin{aligned}
& x=\frac{1}{2 c}\left(-b \beta_{1}^{2}+b \beta_{1} \beta_{2}\right)-2, \\
& y=\frac{1}{2 c}\left(b \beta_{1}^{2}-b \beta_{1} \beta_{2}\right)+1, \\
& z=-a \beta_{1}+b \beta_{1}^{2} Q+2 c Q+c_{c}+c_{R 1} \\
& d=\frac{1}{2 c}\left(b \beta_{2}^{2}-b \beta_{1} \beta_{2}\right)+1, \\
& e=\frac{1}{2 c}\left(-b \beta_{2}^{2}+b \beta_{1} \beta_{2}\right)-2, \\
& f=-a \beta_{2}+b \beta_{2}^{2} Q+2 c Q+c_{e}+c_{R 2}-T \beta_{2}-W .
\end{aligned}
$$

The NE can be found by simultaneously solving the FOC of the refineries' profit function given the BR of the farmer in domain 2. 
Proposition 2.7 In the presence of food market and with the farmer's increasing marginal cost structure, the NE of refineries in domains 3-7 are as follows:

i) In domain $3, p_{2}^{*}=\frac{(c+l)\left(a \beta_{2} c+k-c c_{R 2}-c c_{e}+c T \beta_{2}+c W\right)+b \beta_{2}^{2} k}{b \beta_{2}^{2} c+2 c(c+l)}-c_{e}+W, p_{1}^{*}<p_{2}^{*}$

ii) In domain $4, p_{1}^{*}=\frac{(c+l)\left(a \beta_{1} c+k-c c_{R 1}-c c_{c}\right)+b \beta_{1}^{2} k}{b \beta_{1}^{2} c+2 c(c+l)}-c_{c}, p_{2}^{*}<p_{1}^{*}$

iii) In domain $5, p_{2}^{*}=a \beta_{2}-b \beta_{2}^{2} Q-c_{R 2}+T \beta_{2}, p_{1}^{*}<p_{2}^{*}$

iv) In domain $6, p_{1}^{*}=a \beta_{1}-b \beta_{1}^{2} Q-c_{R 1}, p_{2}^{*}<p_{12}^{*}$

v) In domainn $7, p_{1}^{*}-c+p_{2}^{*}-c_{e}+W \leq \frac{-\left(\left(a_{0}-c_{c}\right) c-2 c Q\left(b_{0}+c\right)\right)}{\left(b_{0}+c\right)}$

The NE can be found by simultaneously solving the FOC of the refineries' profit function given the BR of the farmer in domains 3-7. In domain 7, price offers are too low (lower than the given threshold) such that the farmer will not allocate any land to the refineries. The threshold is given in the Proposition.

Using the results from domain 1, in which all the farmer's decision variables are positive, we can numerically study the effect of subsidies on the performance of the BSC. $p_{1}$ is increased with the increase of both $W$ and $T . p_{2}$ is decreased with an increase of $W$, but not with an increase of $T$. The increase of both $W$ and $T$ decreases the land allocated to the food market and R1, while it increases the land allocated to R2. Figure 2.11 shows the trend of profits under various subsidy plans. From Figure 2.11(a), we can infer that the farmer gets benefit from the subsidy plans; however, her average profit does not show a significant change from the previous case (with food market and linear cost structure) despite her increasing marginal cost, which reveals the advantage of the food market for the farmer. Both subsidies have a negative effect on the profit of R1, but on average, R1 gets the highest profit in this case among all cases. R2 will get advantage of the farmer's increasing marginal cost, which can be confirmed by the increase of its average profit from previous case (with food market and linear cost structure). Although we do not observe the 
subsidy being offset in this case unlike Case 3 (Figure 2.12), it is only in this case where the CS is increased by the payment of subsidy, which makes this case as the most consumer-friendly one (i.e., the Tot. CS. Inc. is positive).

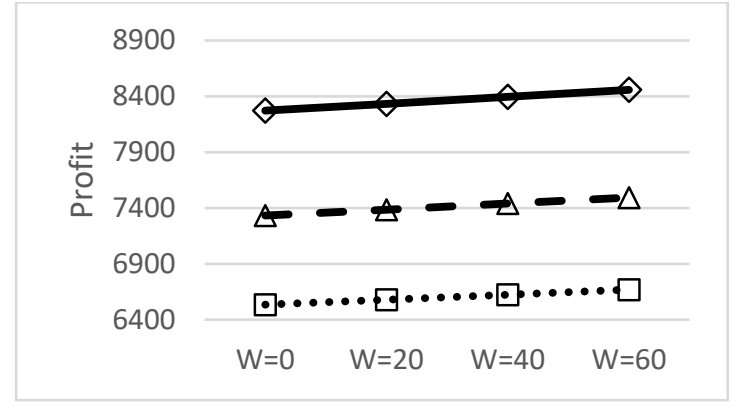

(a) Profit of the farmer

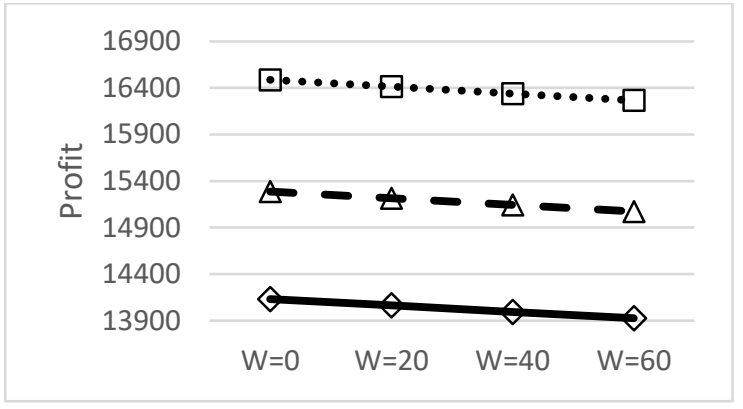

(b) Profit of corn-based refinery (R1)

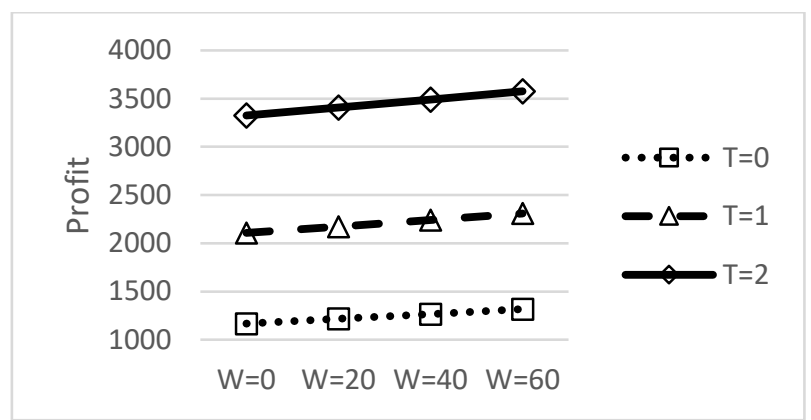

(c) Profit of energy crop-based refinery (R2)

Figure 2.11 Profit of the farmer and refineries under various subsidy policies (Case 4)

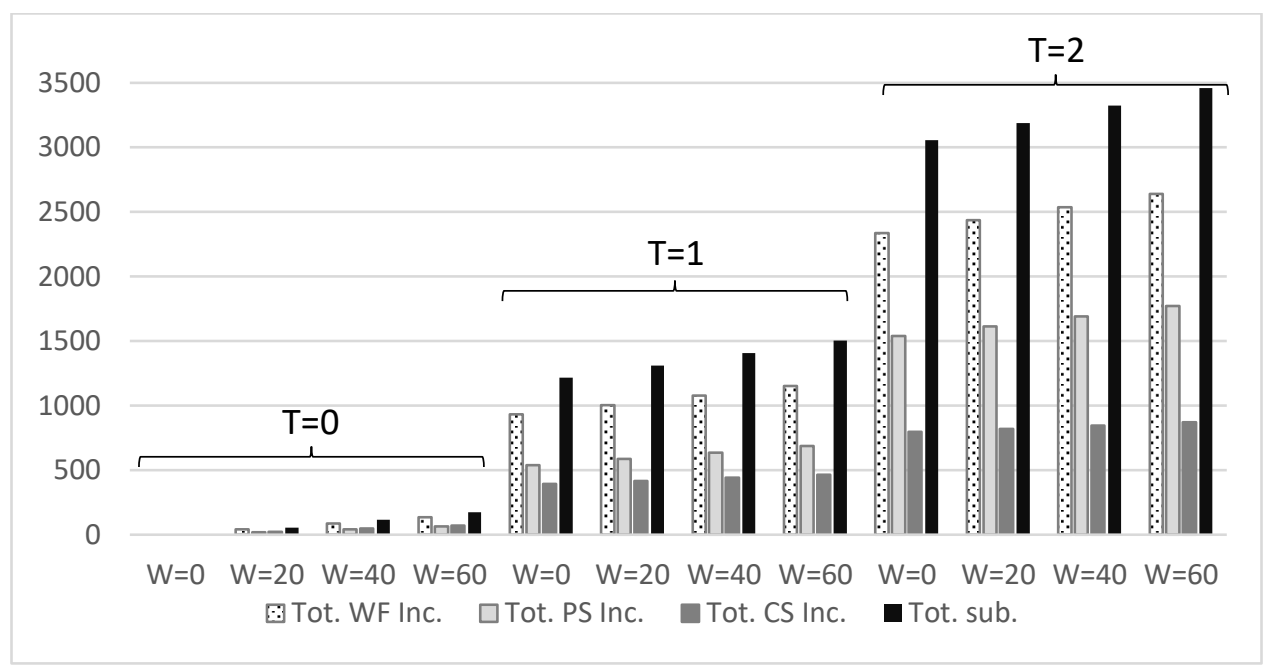

Figure 2.12 Total SW increase breakdown under various policies vs. subsidy paid (Case 4)

Tot. $\mathrm{SW}$ Inc. $=$ Total $\mathrm{SW}$ increase from the $(\mathrm{W}=0, \mathrm{~T}=0)$ policy

Tot. PS Inc. $=$ Total PS increase from the $(\mathrm{W}=0, \mathrm{~T}=0)$ policy

Tot. CS Inc. $=$ Total CS increase from the $(\mathrm{W}=0, \mathrm{~T}=0)$ policy 


\subsection{Policy Analysis}

In this section, we analyze whether government's subsidy policy is capable enough to boost the production of energy-crop biofuel. Considering the EPA's goal for 2022 to produce 36 BG of biofuel, out of which 21 BG should be advanced biofuel, we set the ratio of corn biofuel production to energy-crop biofuel production (ratio $=\beta_{1} q_{1} / \beta_{2} q_{2}$ ) as a performance measure of a given policy $(W, T)$. Considering the EPA's mandate, ratio needs to be less than or equal to 0.714 . In Cases 1 and 3 where we assume a constant marginal cost structure for the farmer as well as the indifference of the farmer between refineries when the marginal profit of both crops are the same, the ratio would be equal to one of these values $\{0,0.5$, Infinity $\}$ since either of the refineries will take all the land or they will share the land equally. However, in Cases 2 and 4, where the farmer has increasing marginal cost, the ratio can take any value in the range ( 0 , infinity) for a given policy. So, for Cases 2 and 4, we find the policy in which the ratio is equal to the EPA's goal (0.714) and imposes the lowest cost to the government.

Using a parametric analysis, the ratio was found for a continuous range of $W$ and a discrete set of $T$ with an increment of $\$ 0.1$ per gallon as illustrated in Figure 2.13. The parallel lines in Figure 2.13 represent the value of ratio at different levels of $T$ and $W$. The horizontal line (Limit) is the goal ratio (0.71), and as it can be observed in both figures, it is achievable by more than one possible policy. The candidate policies to achieve the goal ratio are $\left(W^{*}, T^{*}\right)=\{(94,1.6),(47,1.7)\}$ and $\left(W^{*}, T^{*}\right)=\{(36,2.8),(92,2.7)\}$ for Cases 2 and 4 respectively. A counter-intuitive result from the comparison of the total budget expenditure between the candidate policies within each case shows that the same amount of budget is distributed between the farmer and the refinery to get the same supply chain performance measures (i.e. same SW, CS, PS). This is an interesting result that shows, among the policies that meet the EPA's mandate, none of them favor the farmer or R2. 


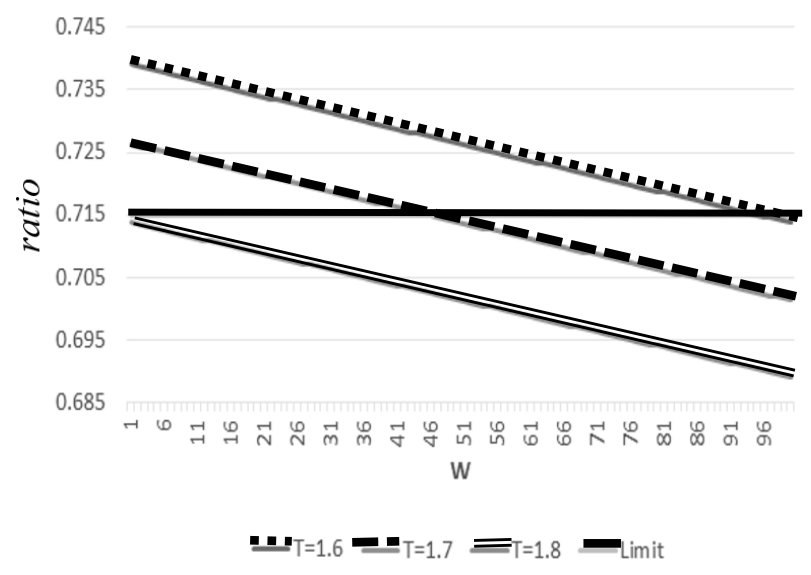

(a) ratio under candidate policies for Case 2

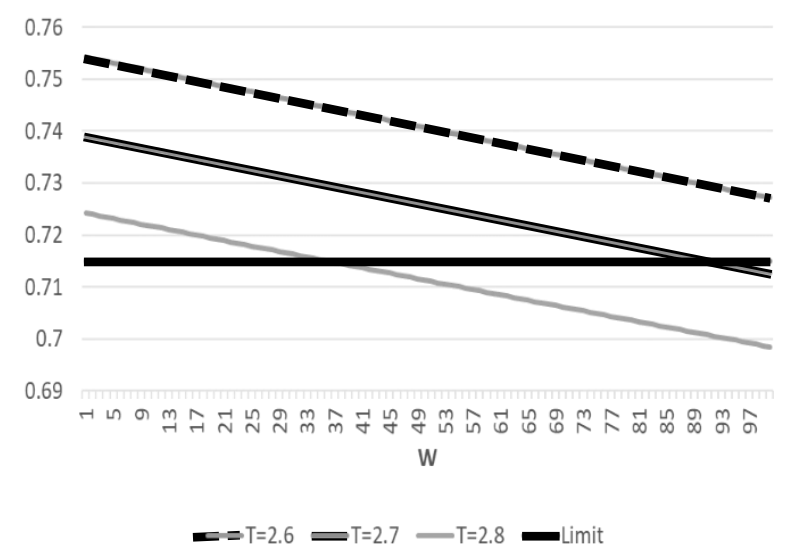

(b) ratio under candidate policies for Case 4

Figure 2.13 Best policy selection in Cases 2 (with corn market) and 4 (without corn market)

\subsection{Conclusion}

In this chapter, we used a game theoretic approach to model a Biofuel Supply Chain (BSC) with a farmer (or a union of farmers), a corn-based refinery (R1), and an energy crop-based refinery (R2) with subsidies paid by the government to the farmer and R 2 to encourage the advanced biofuel production. We studied the problem under four different cases based on the existence of a food market for the farmer as well as having a constant or increasing marginal cost structure and found the equilibrium for each. Furthermore, we performed several parametric analyses using realistic values from the literature.

A profit comparison across the cases was performed for each of the firms showing that the farmer has the highest profit when there is corn market and she has a constant marginal cost structure (i.e. Case 3). In Case 2 (no food market and increasing marginal cost structure), the farmer would not grow any crops unless at higher values of $T$. The increasing marginal cost of the farmer and being bound to selling to the refineries only is the reason for that. In this case, refineries are offering lower prices, taking advantage of the farmer's higher costs. The profit of the farmer is not much sensitive to subsidies in Cases 3 and 4 (with food market and constant/increasing marginal cost), which means the existence of the food market can stabilize the farmer's profit. 
R1 is on average better off at Case 2 (no food market and increasing marginal cost) followed by Case 4 (food market and constant marginal cost) than the other two cases, showing that R1 can get advantage from the increasing marginal cost of the farmer, which helps R1 to be more competitive against the food market and get more land. For these reasons, R1 has the highest profit in these cases. Another insight from the analysis of R1's profit is that in Case 3 (food market and constant marginal cost), R1 can get benefit from the subsidy plans (unlike other cases) while nothing is paid directly to him. It is in Case 4 (food market and increasing marginal cost) (followed by Case 2) where R2 has the highest average profit, showing that the increasing marginal cost of the farmer can be beneficial for R2 as well.

Policy analysis was performed to evaluate the effectiveness of the subsidy budget paid by the government in the increase of advanced biofuel's penetration in the biofuel market. We found that government's intervention through subsidizing the farmer or R2 is effective enough to meet the EPA's mandate of minimum advanced biofuel production. Also, using parametric analyses, we found subsidy policies that meet the EPA mandate and requires the minimum expenditure. An important finding through numerical study was that, in the existence of food market and without the expansion availability (Case 3), the subsidy paid by the government is offset by the increase of the total welfare including supply chain's profit and consumer's surplus, which can be encouraging for governments to establish subsidy plans.

Finally, a parametric analysis shows that the policies with the same performance (i.e. the same value of ratio, defined before) have the same expenditure by the government as well as the same supply chain performance measures (SW, CS, PS) though these policies suggest different distributions of the same budget between the firms. This finding can give policymakers an insight of how a certain amount of budget can be spent in different ways to achieve similar results. 


\section{Chapter 3}

\section{Equilibrium Analysis of a BSC with Constrained Refineries}

\subsection{Introduction}

In Chapter 2, we assumed that the refineries can process any amount of land that the farmer allocates to them, even if they are allocated the entire capacity of the farmer. Although this assumption may be acceptable in a regional scope, we relax this assumption in this chapter for larger farm land. We are interested in finding the equilibria when the refineries have a capacity limit which is known to all firms.

Considering the refineries' capacity constraint can be studied under various conditions. Figure 3.1 shows all possible cases that might happen under the existence of capacity constrain. Domain 5 in Figure 3.1 refers to the cases we already considered in Chapter 1 , in which the refineries have enough capacity to process all the farmer's land. Domain 1 is the case where the total demand of the refineries is less than the total capacity of the farmer. Hence, there will not be any competition between refineries, which makes this domain not interesting to analyze. In domains 3-4, one of the refineries has a capacity larger than that of the farmer, which we do not analyze in this research. Domain 2 is the most interesting case, in which the capacity of the farmer is larger than the capacity of individual refineries, but less than their total capacity.

In this chapter, we represent the four previously discussed models (cases) for domain 2 as it is the most realistic and interesting domain. The capacity of refineries $\left(\bar{q}_{i}\right)$ are passed as upper bounds on land allocation decision variables to the farmer's problem in each case. Backward Induction is used to obtain the equilibria. 


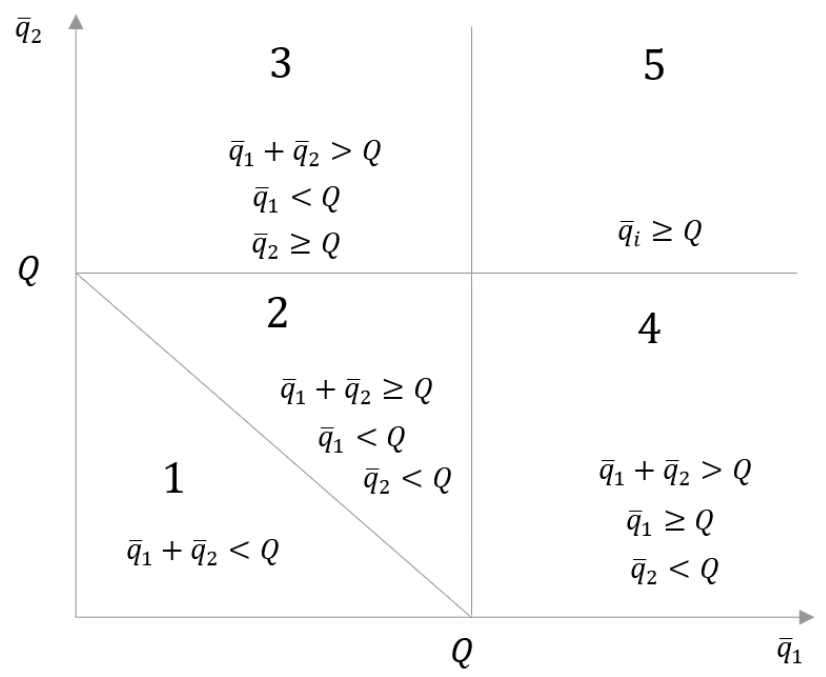

Figure 3.1 Domain of models with capacity constraint

\subsection{Absence of food market}

\subsubsection{Constant marginal cost (Case 1)}

The farmer has the following optimization problem:

$$
\begin{aligned}
& \max _{q_{1}, q_{2}} \pi_{F}=p_{1} q_{1}+p_{2} q_{2}-c_{c} q_{1}-c_{e} q_{2}+W q_{2} \\
& q_{1}+q_{2} \leq Q \\
& 0 \leq q_{1} \leq \bar{q}_{1} \\
& 0 \leq q_{2} \leq \bar{q}_{2}
\end{aligned}
$$

Proposition 3.1 In the absence of food market and with constant marginal cost structure for the capacitated problem, the equilibria are as follows:

i) if $p_{1}^{\max }-c_{c}>p_{2}^{\max }-c_{e}+W, p_{1}^{*}=p_{2}^{\max }-c_{e}+W+c_{c}+\varepsilon, p_{2}^{*}=p_{2}^{\max }$

$$
q_{1}^{*}=\bar{q}_{1}, q_{2}^{*}=Q-\bar{q}_{1}
$$

ii) if $p_{1}^{\max }-c_{c}<p_{2}^{\max }-c_{e}+W, p_{2}^{*}=p_{1}^{\max }-c_{c}+c_{e}-W+\varepsilon, p_{1}^{*}=p_{1}^{\max }$

$$
q_{1}^{*}=Q-\bar{q}_{2}, q_{2}^{*}=\bar{q}_{2}
$$

iii) if $p_{1}^{\max }-c_{c}=p_{2}^{\max }-c_{e}+W, p_{1}^{*}=p_{1}^{\max }, p_{2}^{*}=p_{2}^{\max }$

$$
q_{1}^{*}=q_{2}^{*}=\frac{Q}{2}
$$

In Proposition 3.1, $p_{1}^{\max }=a \beta_{1}-b \beta_{1}^{2} \bar{q}_{1}-b \beta_{1} \beta_{2}\left(Q-\bar{q}_{1}\right)-c_{R 1}$ and $p_{2}^{\max }=a \beta_{2}-b \beta_{2}^{2} \bar{q}_{2}-b \beta_{1} \beta_{2}\left(Q-\bar{q}_{2}\right)-c_{R 2}+T \beta_{2}$ are the highest prices which R1 and R2 are willing to pay while they would make no profit if their maximum 
capacity $\left(\bar{q}_{i}\right)$ is given to them. A comparison between the maximum prices of the capacitated and uncapacitated models show that refineries offer lower prices under the capacitated model, while the total profit of refineries increases. The lower price offer can be justified by their limited operational capacity, and the fact that there would always be some land left for them even if they lose the competition. In contrast, in the uncapacitated model, there is no left-over land for the out priced refinery as the winner gets all the land; however, in the capacitated model, the out priced refinery would get some land even if they offer lower.

In Figure 3.2, the social welfare increase, its components, and the subsidy payment under a few subsidy policies are shown. It is observed that unlike Case 1 in Chapter 2, the advanced biofuel producer can still produce even if he loses the competition. However, as the government increases the subsidy and the advanced refinery wins the competition, the consumer surplus decreases because of the lower biofuel production due to inherent inefficiency of land use of advanced refinery and higher market fuel price.

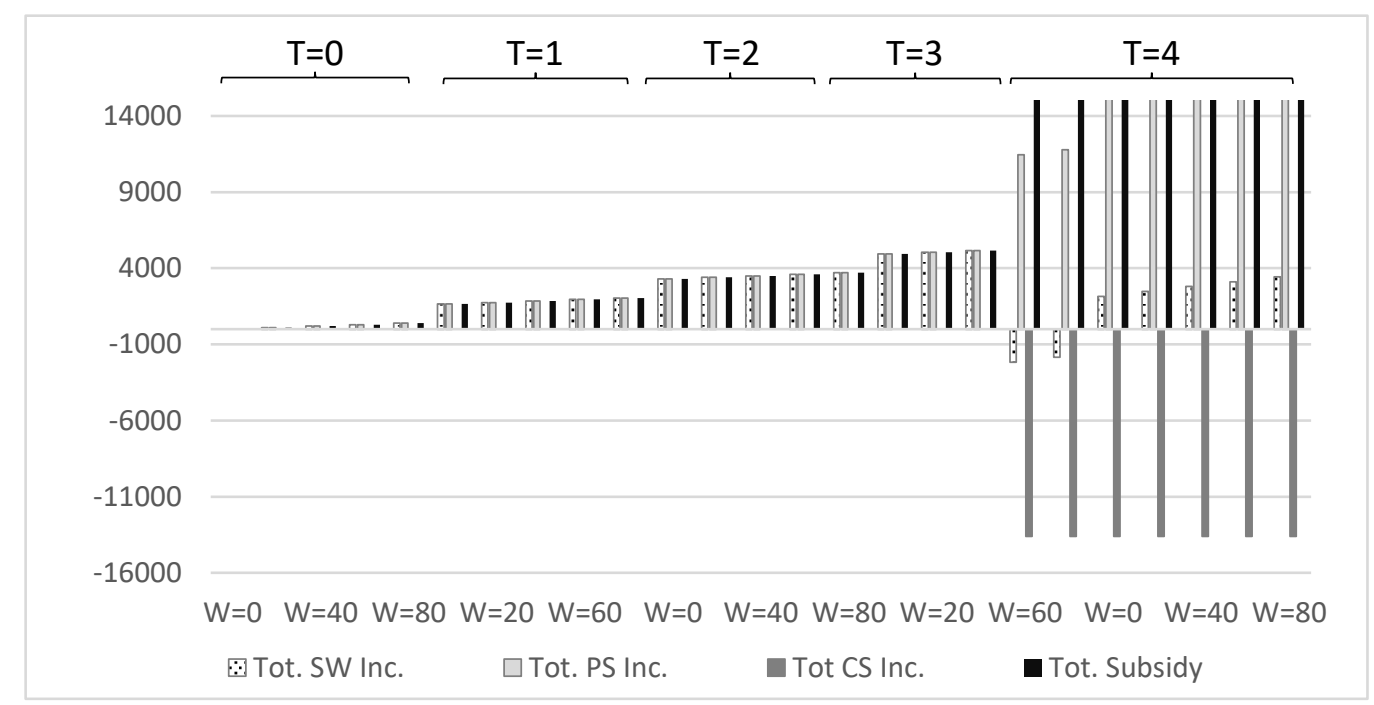

Figure 3.2 Total SW increase breakdown under various policies vs. subsidy paid (Capacitated model-Case 1) Tot. SW Inc. $=$ Total SW increase from the $(\mathrm{W}=0, \mathrm{~T}=0)$ policy

Tot. PS Inc. $=$ Total PS increase from the $(\mathrm{W}=0, \mathrm{~T}=0)$ policy

Tot. CS Inc. $=$ Total CS increase from the $(\mathrm{W}=0, \mathrm{~T}=0)$ policy

\subsubsection{Increasing marginal cost (Case 2)}

With the increasing marginal cost, the farmer's problem is: 
$\max _{q_{1}, q_{2}} \pi_{F}=p_{1} q_{1}+p_{2} q_{2}-\left[c q_{1}^{2}+c_{c} q_{1}\right]-\left[c q_{2}^{2}+c_{e} q_{2}\right]+W q_{2}$

$q_{1}+q_{2} \leq Q \quad\left(\lambda_{3}\right)$

$0 \leq q_{1} \leq \bar{q}_{1} \quad\left(\lambda_{1}, \lambda_{4}\right)$

$0 \leq q_{2} \leq \bar{q}_{2} \quad\left(\lambda_{2}, \lambda_{5}\right)$

This convex optimization problem can be represented by the KKT conditions bellow and solved for the best responses of the farmer.

$$
\begin{aligned}
& L=\pi_{f}+\lambda_{3}\left(Q-q_{1}-q_{2}\right)+\lambda_{1} q_{1}+\lambda_{2} q_{2}+\lambda_{4}\left(\bar{q}_{1}-q_{1}\right)+\lambda_{5}\left(\bar{q}_{2}-q_{2}\right) \\
& \partial L / \partial q_{1}=p_{1}-2 c q_{1}-c_{c}-\lambda_{3}+\lambda_{1}-\lambda_{4}=0 \\
& \partial L / \partial q_{2}=p_{2}-2 c q_{2}-c_{e}+W-\lambda_{3}+\lambda_{2}-\lambda_{5}=0 \\
& 0 \leq \lambda_{1} \perp q_{1} \geq 0 \\
& 0 \leq \lambda_{2} \perp q_{2} \geq 0 \\
& 0 \leq \lambda_{3} \perp Q-q_{1}-q_{2} \geq 0 \\
& 0 \leq \lambda_{4} \perp \bar{q}_{1}-q_{1} \geq 0 \\
& 0 \leq \lambda_{5} \perp \bar{q}_{2}-q_{2} \geq 0
\end{aligned}
$$

We find the domain of offered prices of refineries, including the domains in which the capacity constraint of the farmer is not binding. Figure 3.3 illustrates the domains in terms of $p_{1}^{\prime}=p_{1}-c_{c}$ and $p_{2}^{\prime}=p_{2}+W-c_{e}$. Domain 1 represents a solution where the farmer allocates a positive area of land to both refineries and the capacity constraint of the farmer may or may not be binding. In domain 2, refineries offer higher prices than in domain 1 such that the famer will share the whole capacity between them. In domain 3 , the offered price of R2 is high enough to receive his maximum capacity $\left(\bar{q}_{2}\right)$, but R1 does not offer a high enough price to get all the rest of the capacity, but partially. In domain 4, R1 offers a high price for the farmer's corn such that he receives his maximum capacity $\left(\bar{q}_{1}\right)$ and a part of the rest of the farmer's land is allocated to R2. With a minor difference, domains 5 and 6 are similar to 3 and 4 respectively. In these cases, the 
farmer would allocate all the residual land to the loser refinery. A comparison between this case and Case 2 (no food market and constant marginal cost) in previous section reveals that refineries would have to offer lower prices in the capacitated case due to their lower demand. We show the conditions under which the equilibrium would fall in either of these six domains as well as the corresponding best responses of the farmer and the value of KKT multipliers for those domains in Table 3.1.

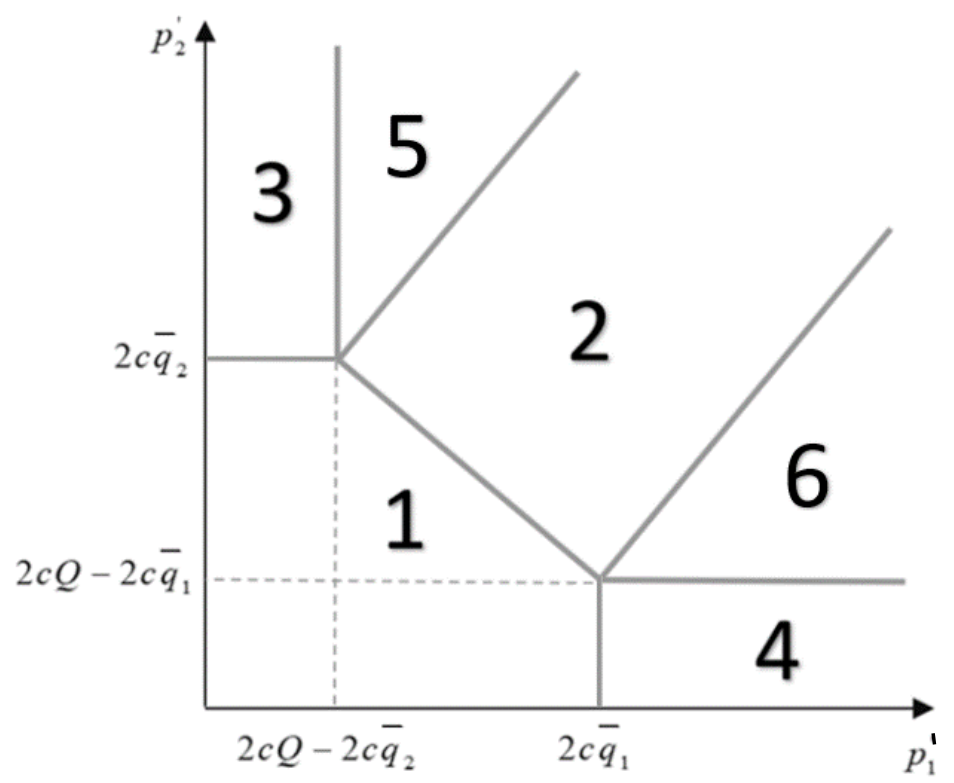

Figure 3.3 Domains of the price offers' space $\left(p_{1}^{\prime}, p_{2}^{\prime}\right)$ in Case 2 of the capacitated model

Table 3.1 Conditions on offered prices and the farmer's BR in Case 2 of the capacitated model

\begin{tabular}{|c|c|c|c|c|}
\hline Domain & $\left(\lambda_{1}, \lambda_{2}, \lambda_{3}, \lambda_{4}, \lambda_{5}\right)$ & Conditions & Farmer's BR & $\lambda_{i}$ \\
\hline 1 & $(0,0,0,0,0)$ & $\begin{array}{l}p_{1}-c_{c} \leq 2 c \bar{q}_{1} \\
p_{2}-c_{e}+W \leq 2 c \bar{q}_{2} \\
p_{1}-c_{c}+p_{2}-c_{e}+W \leq 2 c Q\end{array}$ & $\begin{array}{l}q_{1}^{*}=\frac{p_{1}-c_{c}}{2 c} \\
q_{2}^{*}=\frac{p_{2}-c_{e}+W}{2 c}\end{array}$ & $\lambda_{1}=\lambda_{2}=\lambda_{3}=\lambda_{4}=\lambda_{5}=0$ \\
\hline 2 & $(0,0,+, 0,0)$ & $\begin{array}{l}p_{2}-c_{e}+W+p_{1}-c \geq 2 c Q \\
p_{2}-c_{e}+W-p_{1}+c \leq 4 c \bar{q}_{2}-2 c Q \\
p_{1}-c-p_{2}+c_{e}-W \leq 4 c \bar{q}_{1}-2 c Q\end{array}$ & $\begin{array}{l}q_{1}^{*}=\frac{p_{1}-c_{c}-\lambda_{3}}{2 c} \\
q_{2}^{*}=\frac{p_{2}-c_{e}+W-\lambda_{3}}{2 c}\end{array}$ & $\begin{array}{l}\lambda_{1}=\lambda_{2}=\lambda_{4}=\lambda_{5}=0 \\
\lambda_{3}=\frac{p_{1}^{\prime}+p_{2}^{\prime}-2 c Q}{2}\end{array}$ \\
\hline 3 & $(0,0,0,0,+)$ & $\begin{array}{l}p_{2}-c_{e}+W>2 c \bar{q}_{2} \\
p_{1}-c_{c} \leq 2 c Q-2 c \bar{q}_{2} \\
p_{1}-c_{c} \leq 2 c \bar{q}_{1}\end{array}$ & $\begin{aligned} q_{1}^{*} & =\frac{p_{1}-c_{c}}{2 c} \\
q_{2}^{*} & =\bar{q}_{2}\end{aligned}$ & $\begin{array}{l}\lambda_{1}=\lambda_{2}=\lambda_{3}=\lambda_{4}=0 \\
\lambda_{5}=p_{2}-c_{e}+W-2 c \bar{q}_{2}\end{array}$ \\
\hline
\end{tabular}




$$
\begin{aligned}
& p_{2}-c_{e}+W \leq 2 c \bar{q}_{2} \quad q_{1}^{*}=\bar{q}_{1} \quad \lambda_{1}=\lambda_{2}=\lambda_{3}=\lambda_{5}=0 \\
& 4 \quad(0,0,0,+, 0) \quad p_{2}-c_{e}+W \leq 2 c Q-2 c \bar{q}_{1} \quad q_{2}^{*}=\frac{p_{2}-c_{e}+W}{2 c} \quad \lambda_{4}=p_{1}-c_{c}-2 c \bar{q}_{1} \\
& p_{1}-c_{c}>2 c \bar{q}_{1} \\
& 5 \quad(0,0,+, 0,+) \quad p_{2}^{\prime} \geq 2 c Q-2 c \bar{q} \\
& q^{*}=Q-\bar{q}_{2} \quad \lambda_{1}=\lambda_{2}=\lambda_{4}=0 \\
& q_{2}^{*}=\bar{q}_{2} \quad \lambda_{3}=p_{1}^{\prime}-2 c\left(Q-\bar{q}_{2}\right) \\
& \lambda_{5}=p_{2}^{\prime}-p_{1}^{\prime}+2 c Q-4 c \bar{q}_{2} \\
& 6 \quad(0,0,+,+, 0) \quad p_{1}^{\prime} \geq 2 c Q-2 c \bar{q}_{2} \\
& q_{1}^{*}=\bar{q}_{1} \\
& q_{2}^{*}=Q-\bar{q}_{1} \\
& \lambda_{1}=\lambda_{2}=\lambda_{5}=0 \\
& \lambda_{3}=p_{2}^{\prime}-2 c\left(Q-\bar{q}_{1}\right) \\
& \lambda_{4}=p_{1}^{\prime}-p_{2}^{\prime}+2 c Q-4 c \bar{q}_{1}
\end{aligned}
$$

In Proposition 3.2, we show the NE of domains 1-6.

Proposition 3.2 In the presence of food market and with the farmer's constant marginal cost structure for the capacitated problem, the equilibria are as follows:

i) In domain 1, NE is equivalent to the corresponding conditions on offered prices (Table 3.1)

ii) In domain 2, NE is identical to that of domain 4 in Section 2.3.1.2

iii) In domain $3, p_{1}^{*}=\frac{a \beta_{1} c-b \beta_{1} \beta_{2} \bar{q}_{2} c-c c_{c}-c_{R 1} c}{b \beta_{1}^{2}+2 c}+c_{c}, p_{2}^{*}>2 c \bar{q}_{2}+c_{e}-W$

iv) In domain $4, p_{2}^{*}=\frac{a \beta_{2} c-b \beta_{1} \beta_{2} \bar{q}_{1} c-c c_{e}-c_{R 2} c+T \beta_{2} c}{b \beta_{2}^{2}+2 c}+c_{e}-W, p_{1}^{*}>2 c \bar{q}_{1}+c_{c}$

v) In domain $5, p_{1}^{*}=2 c Q-2 c \bar{q}_{2}+c_{c}, p_{2}^{*}=p_{1}^{*}+c_{e}-W-c_{c}-2 c Q+4 c \bar{q}_{2}+\varepsilon$

$$
q_{1}^{*}=Q-\bar{q}_{2}, q_{2}^{*}=\bar{q}_{2}
$$

vi) In domain $6, p_{2}^{*}=2 c Q-2 c \bar{q}_{1}+c_{e}-W, p_{1}^{*}=p_{2}^{*}-c_{e}+W+c_{c}-2 c Q+4 c \bar{q}_{1}+\varepsilon$

$$
q_{1}^{*}=\bar{q}_{1}, q_{2}^{*}=Q-\bar{q}_{1}
$$

\subsection{Presence of food market}

\subsubsection{Constant marginal cost (Case 3)}

With the presence of food market and the constant marginal cost of the farmer, her problem can be written as: 


$$
\max _{q_{0}, q_{1}, q_{2}} \pi_{F}=p_{1} q_{1}+p_{2} q_{2}-c_{c}\left(q_{0}+q_{1}\right)-c_{e} q_{2}+W q_{2}+\left(a_{0}-b_{0} q_{0}\right) q_{0}
$$

$q_{0}+q_{1}+q_{2} \leq Q \quad\left(\lambda_{3}\right)$

$0 \leq q_{0} \quad\left(\lambda_{6}\right)$

$0 \leq q_{1} \leq \bar{q}_{1} \quad\left(\lambda_{1}, \lambda_{4}\right)$

$0 \leq q_{2} \leq \bar{q}_{2} \quad\left(\lambda_{2}, \lambda_{5}\right)$

This convex optimization problem can be represented by the KKT conditions bellow and solved for the best responses of the farmer.

$$
\begin{aligned}
& L=\pi_{f}+\lambda_{3}\left(Q-q_{1}-q_{2}\right)+\lambda_{1} q_{1}+\lambda_{2} q_{2}+\lambda_{3}\left(Q-q_{0}-q_{1}-q_{2}\right)+\lambda_{4}\left(\bar{q}_{1}-q_{1}\right)+\lambda_{5}\left(\bar{q}_{2}-q_{2}\right)+\lambda_{6} q_{0} \\
& \partial L / \partial q_{0}=-c_{c}+a_{0}-2 b_{0} q_{0}+\lambda_{6}-\lambda_{3}=0 \\
& \partial L / \partial q_{1}=p_{1}-c_{c}-\lambda_{3}+\lambda_{1}-\lambda_{4}=0 \\
& \partial L / \partial q_{2}=p_{2}-c_{e}+W-\lambda_{3}+\lambda_{2}-\lambda_{5}=0 \\
& 0 \leq \lambda_{1} \perp q_{1} \geq 0 \\
& 0 \leq \lambda_{2} \perp q_{2} \geq 0 \\
& 0 \leq \lambda_{3} \perp Q-q_{0}-q_{1}-q_{2} \geq 0 \\
& 0 \leq \lambda_{4} \perp \bar{q}_{1}-q_{1} \geq 0 \\
& 0 \leq \lambda_{5} \perp \bar{q}_{2}-q_{2} \geq 0 \\
& 0 \leq \lambda_{6} \perp q_{0} \geq 0
\end{aligned}
$$

Solving Equations (8)-(16) gives 11 feasible solutions (out of the total 64 possible solutions from the positivity condition of Lagrangian multipliers), for which we find the corresponding domain of offered prices of refineries based on the value of $p_{1}^{\prime}=p_{1}-c_{c}$ and $p_{2}^{\prime}=p_{2}+W-c_{e}$ as shown in Figure 3.4 and summarized in Table 3.2. Domain 1 consists of a solution, in which the capacity constraint of the farmer is binding, and both refineries offer equal marginal profits to the farmer, and they are allocated the same amount of land. However, their offer is not high enough to prevent the farmer from selling her corn to the food market. In domain 2 (3), the farmer would allocate the whole land to the food market and R2 (R1) while the refinery 
would not receive his demanded capacity. In domain 4 (5), R2 (R1) receives his demanded capacity and the surplus land is shared between R1 (R2) and to the food market. In domain 6, all the farmer's capacity is shared between the refineries.

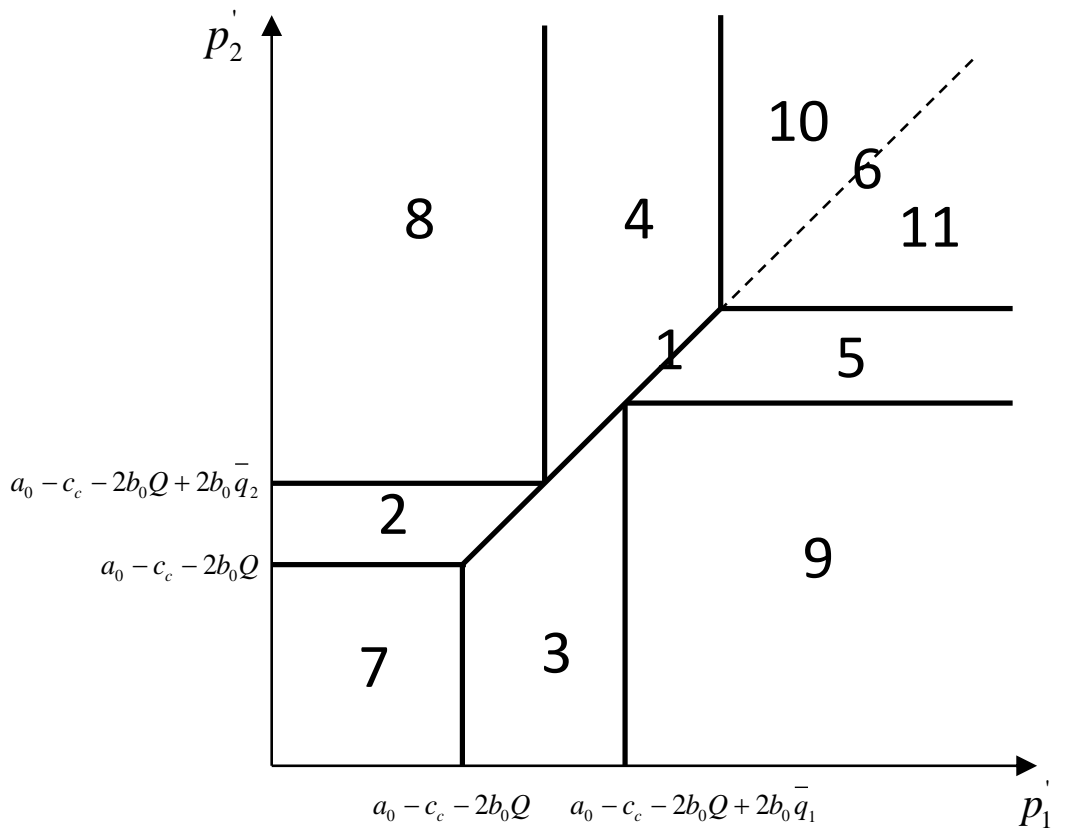

Figure 3.4 Domains of the price offers' space $\left(p_{1}^{\prime}, p_{2}^{\prime}\right)$ in Case 3 of the capacitated model

Figure 3.4 is not symmetric because of the different capacity of the refineries. Here we assume refineries don't have equal capacity to show its effect on the domains. In domain 7, the refineries' offered prices are too low, and the farmer would sell only to the food market. In domain 8 (9), R2 (R1) receives his demanded capacity and the surplus land would be allocated to the food market. In domain 10 (11), R2 (R1) receives his demanded capacity and the surplus land would be allocated to the R1 (R2). 
Table 3.2 Conditions on offered prices and the farmer's BR in Case 3 of the capacitated model

Domain $\left(\lambda_{1}, \lambda_{2}, \lambda_{3}, \lambda_{4}, \lambda_{5}, \lambda_{6}\right)$

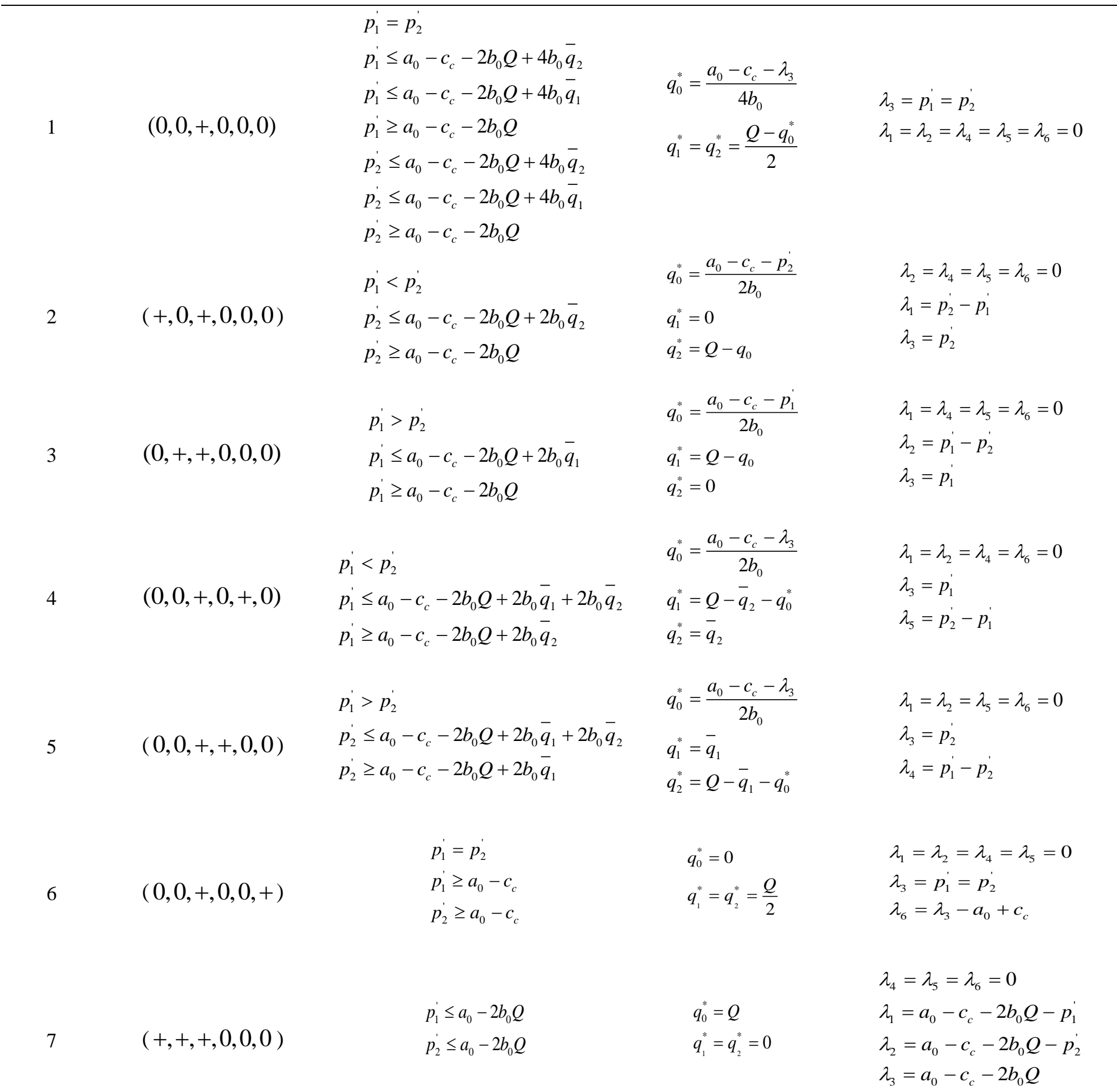


8

9

10

11

$$
p_{1}^{\prime} \leq a_{0}-c_{c}-2 b_{0} Q+2 b_{0} \bar{q}_{2}
$$$$
(+, 0,+, 0,+, 0)
$$

$(0,+,+,+, 0,0)$

$p_{1}^{\prime} \geq 0$

$p_{2}^{\prime}>p_{1}^{\prime}$
$(0,0,+, 0,+,+)$

$$
\begin{aligned}
& q_{0}^{*}=\frac{a_{0}-c_{c}-\lambda_{3}}{2 b_{0}} \\
& q_{1}^{*}=0 \\
& q_{2}^{*}=\bar{q}_{2}
\end{aligned}
$$$$
q_{0}^{*}=\frac{a_{0}-c_{c}-\lambda_{3}}{2 b_{0}}
$$$$
q_{1}^{*}=\bar{q}_{1}
$$$$
q_{2}^{*}=0
$$$$
p_{2}^{\prime} \geq 0
$$$$
(0,0,+,+, 0,+)
$$

$p_{1}^{\prime}>p_{2}^{\prime}$$$
q_{0}^{*}=0
$$$$
q_{1}^{*}=Q-\bar{q}_{2}
$$$$
q_{2}^{*}=\bar{q}_{2}
$$$$
q_{0}^{*}=0
$$

$\lambda_{2}=\lambda_{4}=\lambda_{6}=0$

$\lambda_{1}=\lambda_{3}-p_{1}^{\prime}$

$\lambda_{3}=a_{0}-c_{c}-2 b_{0} Q+2 b_{0} \bar{q}_{2}$

$\lambda_{5}=p_{2}^{\prime}-\lambda_{3}$

$\lambda_{1}=\lambda_{5}=\lambda_{6}=0$

$\lambda_{2}=\lambda_{3}-p_{2}^{\prime}$

$\lambda_{3}=a_{0}-c_{c}-2 b_{0} Q+2 b_{0} \bar{q}_{1}$

$\lambda_{4}=p_{1}^{\prime}-\lambda_{3}$

$$
\begin{aligned}
& \lambda_{1}=\lambda_{2}=\lambda_{4}=0 \\
& \lambda_{3}=p_{1}^{\prime} \\
& \lambda_{5}=p_{2}^{\prime}-p_{1}^{\prime} \\
& \lambda_{6}=p_{1}^{\prime}-a_{0}+c_{c} \\
& \lambda_{1}=\lambda_{2}=\lambda_{5}=0 \\
& \lambda_{3}=p_{2}^{\prime} \\
& \lambda_{4}=p_{1}^{\prime}-p_{2}^{\prime}
\end{aligned}
$$$$
q_{2}^{*}=Q-\bar{q}_{1}
$$

\subsubsection{Increasing marginal cost (Case 4)}

With the increasing marginal cost, the farmer's problem can be written as:

$$
\begin{array}{ll}
\max _{q_{0}, q_{1}, q_{2}} \pi_{F}=p_{1} q_{1}+p_{2} q_{2}-\left[c\left(q_{1}^{2}+q_{0}^{2}\right)+c_{c}\left(q_{1}+q_{0}\right)\right]-\left[c q_{2}^{2}+c_{e} q_{2}\right]+W q_{2}+\left(a_{0}-b_{0} q_{0}\right) q_{0} \\
q_{0}+q_{1}+q_{2} \leq Q & \left(\lambda_{3}\right) \\
0 \leq q_{0} & \left(\lambda_{6}\right) \\
0 \leq q_{1} \leq \bar{q}_{1} & \left(\lambda_{1}, \lambda_{4}\right) \\
0 \leq q_{2} \leq \bar{q}_{2} & \left(\lambda_{2}, \lambda_{5}\right)
\end{array}
$$

This convex optimization problem can be represented by the KKT conditions bellow and solved for the best responses of the farmer.

$$
\begin{aligned}
& L=\pi_{f}+\lambda_{3}\left(Q-q_{1}-q_{2}\right)+\lambda_{1} q_{1}+\lambda_{2} q_{2}+\lambda_{3}\left(Q-q_{0}-q_{1}-q_{2}\right)+\lambda_{4}\left(\bar{q}_{1}-q_{1}\right)+\lambda_{5}\left(\bar{q}_{2}-q_{2}\right)+\lambda_{6} q_{0} \\
& \partial L / \partial q_{0}=a_{0}-2 c q_{0}-c_{c}-2 b_{0} q_{0}-\lambda_{3}+\lambda_{6}=0 \\
& \partial L / \partial q_{1}=p_{1}-2 c q_{1}-c_{c}-\lambda_{3}+\lambda_{1}-\lambda_{4}=0
\end{aligned}
$$


$\partial L / \partial q_{2}=p_{2}-2 c-c_{e}+W-\lambda_{3}+\lambda_{2}-\lambda_{5}=0$

$0 \leq \lambda_{1} \perp q_{1} \geq 0$

$0 \leq \lambda_{2} \perp q_{2} \geq 0$

$0 \leq \lambda_{3} \perp Q-q_{0}-q_{1}-q_{2} \geq 0$

$0 \leq \lambda_{4} \perp \bar{q}_{1}-q_{1} \geq 0$

$0 \leq \lambda_{5} \perp \bar{q}_{2}-q_{2} \geq 0$

$0 \leq \lambda_{6} \perp q_{0} \geq 0$

Figure 3.5 illustrates the domain of all offered prices of refineries based on the value of $p_{1}^{\prime}=p_{1}-c_{c}$ and $p_{2}^{\prime}=p_{2}+W-c_{e}$. The best responses of the farmer along with the necessary conditions on the offered prices and the value of KKT multipliers of each domain are summarized in Table 3.3.

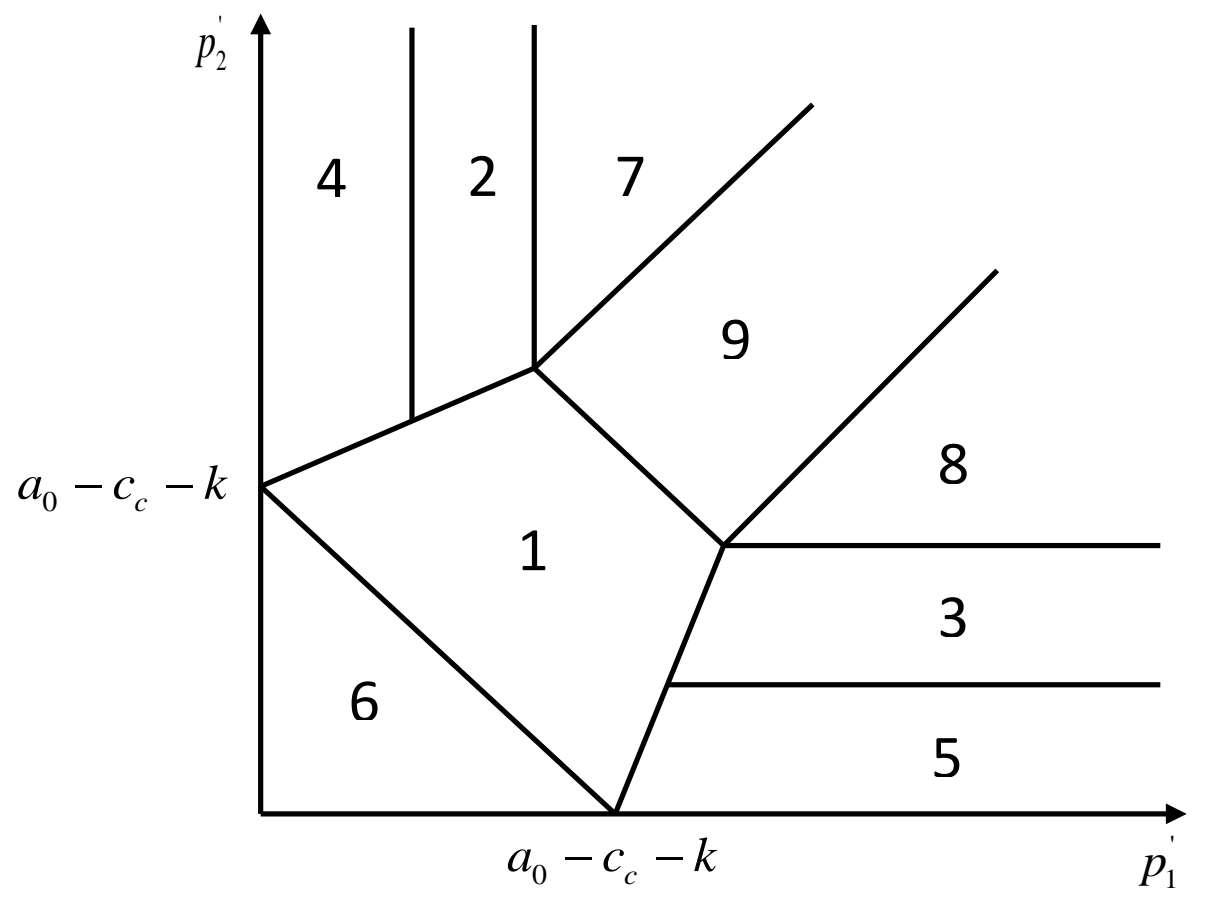

Figure 3.5 Domains of the price offers' space $\left(p_{1}^{\prime}, p_{2}^{\prime}\right)$ in Case 4 of the capacitated model 
Table 3.3 Conditions on offered prices and the farmer's BR in Case 4 of the capacitated model

\begin{tabular}{|c|c|c|c|c|}
\hline Domain & $\left(\lambda_{1}, \lambda_{2}, \lambda_{3}, \lambda_{4}, \lambda_{5}, \lambda_{6}\right)$ & Conditions & Farmer's BR & $\lambda_{i}$ \\
\hline 1 & $(0,0,+, 0,0,0)$ & $\begin{array}{l}l p_{1}^{\prime}+l p_{2}^{\prime} \leq a_{0}-c_{c}-k \\
l p_{1}^{\prime}+l p_{2}^{\prime}>-k \\
l p_{1}^{\prime}+l p_{2}^{\prime}>a_{0}-c_{c}-2 b_{0} Q-2 c Q-k \\
(l-1) p_{1}^{\prime}+l p_{2}^{\prime} \leq-k \\
l p_{1}^{\prime}+(l-1) p_{2}^{\prime} \leq-k \\
(1-l) p_{1}^{\prime}-l p_{2}^{\prime} \leq 2 c \bar{q}_{1}+k \\
-l p_{1}^{\prime}+(1-l) p_{2}^{\prime} \leq 2 c \bar{q}_{2}+k\end{array}$ & $\begin{array}{l}q_{0}^{*}=\frac{a_{0}-c_{c}-\lambda_{3}}{4 b_{0}} \\
q_{1}^{*}=\frac{(1-l) p_{1}^{\prime}-l p_{2}^{\prime}-k}{2 c} \\
q_{2}^{*}=\frac{(1-l) p_{2}^{\prime}-l p_{1}^{\prime}-k}{2 c}\end{array}$ & $\begin{array}{l}\lambda_{3}=k+l p_{1}^{\prime}+l p_{2}^{\prime} \\
\lambda_{1}=\lambda_{2}=\lambda_{4}=\lambda_{5}=\lambda_{6}=0\end{array}$ \\
\hline 2 & $(0,0,+, 0,+, 0)$ & $\begin{array}{l}p_{1} \leq \frac{\left(a_{0}-c_{c}\right)\left(c+b_{0}\right)}{c+b_{0}}-2 c\left(\bar{q}_{2}-Q\right) \\
p_{1} \leq c\left(a_{0}-c_{c}\right)+2 c\left(b_{0}+c\right)\left(\bar{q}_{2}-Q\right)+2 c \bar{q}_{1}\left(2 c+b_{0}\right) \\
\left(2 c+b_{0}\right) p_{2}-\left(b_{0}+c\right) p_{1}^{\prime}>c\left(a_{0}-c_{c}\right)+2 c\left(b_{0}+c\right)\left(\bar{q}_{2}-Q\right)+2 c \bar{q}_{2}\left(2 c+b_{0}\right) \\
p_{1} \geq \frac{\left(2 c+b_{0}\right)\left(a_{0}-c_{c}-2 b_{0} Q-2 c Q\right)-c\left(a_{0}-c_{c}\right)}{c+b_{0}}-2 c\left(\bar{q}_{2}-Q\right) \\
p_{1} \geq c\left(a_{0}-c_{c}\right)+2 c\left(b_{0}+c\right)\left(\bar{q}_{2}-Q\right) \\
p_{1}>2 c Q-2 c \bar{q}_{2}-\frac{c\left(a_{0}-c_{c}\right)}{b_{0}+c}\end{array}$ & $\begin{aligned} q_{0}^{*} & =\frac{a_{0}-c_{c}-\lambda_{3}}{2 b_{0}} \\
q_{1}^{*} & =\frac{p_{1}^{\prime}-\lambda_{3}}{2 c} \\
q_{2}^{*} & =\bar{q}_{2}\end{aligned}$ & $\begin{array}{l}\lambda_{1}=\lambda_{2}=\lambda_{4}=\lambda_{6}=0 \\
\lambda_{3}=\frac{c\left(a_{0}-c_{c}\right)+\left(b_{0}+c\right)\left(p_{1}^{\prime}+2 c \bar{q}_{2}-2 c Q\right)}{b_{0}+2 c} \\
\lambda_{5}=p_{2}^{\prime}-\lambda_{3}-2 c \bar{q}_{2}\end{array}$ \\
\hline 3 & $(0,0,+,+, 0,0)$ & $\begin{array}{l}p_{2} \leq \frac{\left(a_{0}-c_{c}\right)\left(c+b_{0}\right)}{c+b_{0}}-2 c\left(\bar{q}_{1}-Q\right) \\
p_{2}^{\prime} \leq c\left(a_{0}-c_{c}\right)+2 c\left(b_{0}+c\right)\left(\bar{q}_{1}-Q\right)+2 c \bar{q}_{2}\left(2 c+b_{0}\right) \\
\left(c+b_{0}\right) p_{2}^{\prime}-\left(b_{0}+2 c\right) p_{1}^{\prime}<-c\left(a_{0}-c_{c}\right)-2 c\left(b_{0}+c\right)\left(\bar{q}_{1}-Q\right)-2 c \bar{q}_{1}\left(2 c+b_{0}\right) \\
p_{2} \geq \frac{\left(2 c+b_{0}\right)\left(a_{0}-c_{c}-2 b_{0} Q-2 c Q\right)-c\left(a_{0}-c_{c}\right)}{c+b_{0}}-2 c\left(\bar{q}_{1}-Q\right) \\
p_{2} \geq c\left(a_{0}-c_{c}\right)+2 c\left(b_{0}+c\right)\left(\bar{q}_{1}-Q\right) \\
p_{2}>2 c Q-2 c \bar{q}_{1}-\frac{c\left(a_{0}-c_{c}\right)}{b_{0}+c}\end{array}$ & $\begin{array}{l}q_{0}^{*}=\frac{a_{0}-c_{c}-\lambda_{3}}{2 b_{0}} \\
q_{1}^{*}=\bar{q}_{1} \\
q_{2}^{*}=\frac{p_{2}^{\prime}-\lambda_{3}}{2 c}\end{array}$ & $\begin{array}{l}\lambda_{1}=\lambda_{2}=\lambda_{5}=\lambda_{6}=0 \\
\lambda_{3}=\frac{c\left(a_{0}-c_{c}\right)+\left(b_{0}+c\right)\left(p_{2}^{\prime}+2 c \bar{q}_{1}-2 c Q\right)}{b_{0}+2 c} \\
\lambda_{4}=p_{1}^{\prime}-\lambda_{3}-2 c \bar{q}_{1}\end{array}$ \\
\hline 4 & $(+, 0,+, 0,+, 0)$ & $\begin{array}{l}p_{1}^{\prime}<a_{0}-c_{c}-2\left(b_{0}+c\right)\left(Q-\bar{q}_{2}\right) \\
p_{2}^{\prime}>a_{0}-c_{c}-2\left(b_{0}+c\right)\left(Q-\bar{q}_{2}\right)+2 c \bar{q}_{2}\end{array}$ & $\begin{array}{l}q_{0}^{*}=\frac{a_{0}-c_{c}-\lambda_{3}}{2 b_{0}} \\
q_{1}^{*}=0 \\
q_{2}^{*}=\bar{q}_{2}\end{array}$ & $\begin{array}{l}\lambda_{2}=\lambda_{4}=\lambda_{6}=0 \\
\lambda_{1}=\lambda_{3}-p_{1}^{\prime} \\
\lambda_{3}=a_{0}-c_{c}-2\left(b_{0}+c\right)\left(Q-\bar{q}_{2}\right) \\
\lambda_{5}=p_{2}^{\prime}-\lambda_{3}-2 c \bar{q}_{2}\end{array}$ \\
\hline 5 & $(0,+,+,+, 0,0)$ & $\begin{array}{l}p_{1}^{\prime}>a_{0}-c_{c}-2\left(b_{0}+c\right)\left(Q-\bar{q}_{1}\right)+2 c \bar{q}_{1} \\
p_{2}^{\prime}<a_{0}-c_{c}-2\left(b_{0}+c\right)\left(Q-\bar{q}_{1}\right)\end{array}$ & $\begin{aligned} q_{0}^{*} & =\frac{a_{0}-c_{c}-\lambda_{3}}{2 b_{0}} \\
q_{1}^{*} & =\bar{q}_{1} \\
q_{2}^{*} & =0\end{aligned}$ & $\begin{array}{l}\lambda_{1}=\lambda_{5}=\lambda_{6}=0 \\
\lambda_{2}=\lambda_{3}-p_{2}^{\prime} \\
\lambda_{3}=a_{0}-c_{c}-2\left(b_{0}+c\right)\left(Q-\bar{q}_{1}\right) \\
\lambda_{4}=p_{1}^{\prime}-\lambda_{3}-2 c \bar{q}_{1}\end{array}$ \\
\hline 6 & $(0,0,0,0,0,0)$ & $\begin{array}{l}p_{1}^{\prime} \leq 2 c \bar{q}_{1} \\
p_{2}^{\prime} \leq 2 c \bar{q}_{2} \\
p_{1}^{\prime}+p_{2}^{\prime} \leq 2 c Q-\frac{2 c\left(a_{0}-c_{c}\right)}{2 b_{0}+2 c}\end{array}$ & $\begin{array}{l}q_{0}^{*}=\frac{a_{0}-c_{c}}{2 b_{0}+2 c} \\
q_{1}^{*}=\frac{p_{1}^{\prime}}{2 c} \\
q_{2}^{*}=\frac{p_{2}^{\prime}}{2 c}\end{array}$ & $\lambda_{i}=0$ \\
\hline 7 & $(0,0,+, 0,+,+)$ & $\begin{array}{l}p_{1}^{\prime}>a_{0}-c_{c}+2 c\left(Q-\bar{q}_{2}\right) \\
p_{2}^{\prime}-p_{1}^{\prime}>4 c \bar{q}_{2}-2 c Q\end{array}$ & $\begin{array}{l}q_{0}^{*}=0 \\
q_{1}^{*}=\frac{p_{1}^{\prime}-\lambda_{3}}{2 c} \\
q_{2}^{*}=\bar{q}_{2}\end{array}$ & $\begin{array}{l}\lambda_{1}=\lambda_{2}=\lambda_{4}=0 \\
\lambda_{3}=p_{1}^{\prime}-2 c\left(Q-\bar{q}_{2}\right) \\
\lambda_{5}=p_{2}^{\prime}-\lambda_{3}-2 c \bar{q}_{2} \\
\lambda_{6}=\lambda_{3}-a_{0}+c_{c}\end{array}$ \\
\hline
\end{tabular}


$8 \quad(0,0,+,+, 0,+)$

$$
\begin{aligned}
& q_{0}^{*}=0 \\
& q_{1}^{*}=\bar{q}_{1} \\
& q_{2}^{*}=\frac{p_{2}^{\prime}-\lambda_{3}}{2 c}
\end{aligned}
$$$$
\lambda_{1}=\lambda_{2}=\lambda_{5}=0
$$$$
\lambda_{3}=p_{2}^{\prime}-2 c\left(Q-\bar{q}_{1}\right)
$$$$
\lambda_{4}=p_{1}^{\prime}-\lambda_{3}-2 c \bar{q}_{1}
$$$$
\lambda_{6}=\lambda_{3}-a_{0}+c_{c}
$$ 


\section{Chapter 4 \\ Equilibrium analysis of a bilevel BSC with EPEC approach}

\subsection{Introduction}

In this chapter, we generalize the model introduced in Chapter 2 as a bilevel game theoretic model to consider multiple refineries and study the effect of incentives in a larger scale. Our main objective, is to find the effect of the incentives (subsidy) which are required to overcome the financial obstacles to the goals of RFS in a biofuel supply chain problem and to promote the production of advanced biofuel. In addition, we aim to show the effect of the establishment of new biofuel producers (refineries) and their competition on the wellbeing of the society and the subsidy requirement settings.

Decision making on farmland allocation and biofuel production has been previously studied in the literature; however, the existing works fail to integrate the considered factors, players and the socioeconomic impacts of the supply chain, at the same time. In one of the most relevant works, Luo and Miller (2013) calculate the incentives required to stimulate the advanced biofuel production. However, they consider the problem under a simplified framework, and do not study the socio-economic impact of subsidies on the biofuel supply chain. In this chapter, we develop an integrated model to fill the existing gap and study the socio-economic aspects of a biofuel supply chain along with the land use and feedstock pricing decisions. We also consider a spatial characteristic for the model, which allows us to consolidate the transportation costs imposed to refineries and farmers in their decisions accordingly.

In a bilevel (leader-follower) game, we usually have an optimization problem embedded as constraints in another optimization problem. In case we can rewrite the inner (lower level) optimization problem in the form of equivalent variational inequalities or KKT conditions, we can transform the bilevel problem into a single level optimization problem that consists of equilibrium constraints (e.g., complementarity 
conditions). Because of the existence of these constraints, such single level problems are called mathematical programs with equilibrium constraints (MPEC) (Luo et al. 1996). MPECs have been extensively studied and used in many different applications and industries including energy, transportation and production. Examples include Koh (2012), Allevi et al. (2018), and Siddiqui and Christensen (2016). MPECs are non-linear programming (NPL), and special algorithms have been developed to solve them. Among all, the most well-known algorithm is developed by Ferris and Munson (2000), which is implemented in the PATH solver of GAMS.

In our biofuel supply chain, refineries are competing for the farmer's land, and each refinery solves a bilevel problem in which the refinery (leader) maximizes his profit at the upper level problem and the farmer (follower) maximizes her profit at the lower level problem. Because of the convexity of the farmer's problem, we can replace it with its KKT conditions and include them in the refineries' problems as new constraints and solve the resultant single level MPEC. Having derived the MPEC for each of the refineries, we need to jointly consider all MPECs of refineries to obtain the generalized Nash equilibrium (GNE), of the supply chain on which we can perform several analyses. For that, we obtain the KKT conditions of each single level problem and combine them into one single optimization problem. The new problem is called equilibrium problems with equilibrium constraints (EPEC), which has been previously addressed in other works and industries, especially electricity market (e.g. Pozo, \& Contreras 2011; Ruiz et al. 2012 and Kazempour et al. 2013), but not in biofuel supply chain study.

The rest of the chapter has been structured as follows. Section 4.2 presents the problem description, assumptions, notation and mathematical formulation in the form of MPEC and EPEC. The solution methodology has been discussed in section 4.3 followed by a numerical example of the model in section 4.4. We conclude the chapter in section 4.5. 


\subsection{Bilevel biofuel supply chain}

\subsubsection{Problem description}

A network representation of the considered biofuel supply chain is shown in Figure 4.1. The farmer can grow either the conventional crop (corn), an energy-crop or both on her land. Corn can be sold either to a corn market or to conventional refineries, while energy-crop can be sold only to advanced refineries as there is no significant other market for this type of crop. The government subsidizes the farmer and advanced refineries to help overcoming their lower efficiency, promote energy-crop production, and more environment friendly way of biofuel production. It announces the subsidy values (dollars per unit quantity) publicly. Knowing the subsidy, all refineries quote their price $\left(p_{i}\right)$ to the farmer for corn and energy crop, respectively, at the beginning of the growing season to secure their raw material, and then the farmer makes decisions on the use of her land for corn or other energy-crop $\left(q_{i}\right)$ to maximize her profit. We assume a linear price function in the form of $\mathrm{p}()=.\mathrm{a}-\mathrm{b}($.$) for both corn and biofuel markets, to which the farmer and$ refineries sell their products, respectively. The output of all refineries is homogenous and can be sold at the same biofuel market. The biofuel price is determined by the total realized biofuel production supplied to the biofuel market.

The following sets are defined for the formulation of the problem. Set $J=\{1, \ldots, \bar{J}\}$ is the set of conventional corn based refineries, set $K=\{\bar{J}+1, \ldots, \bar{I}\}$ is the set of advanced refineries, and set $I=\{1, \ldots, \bar{I}\}$ is the union of these two sets. Table 4.1 lists more parameters and variables. 


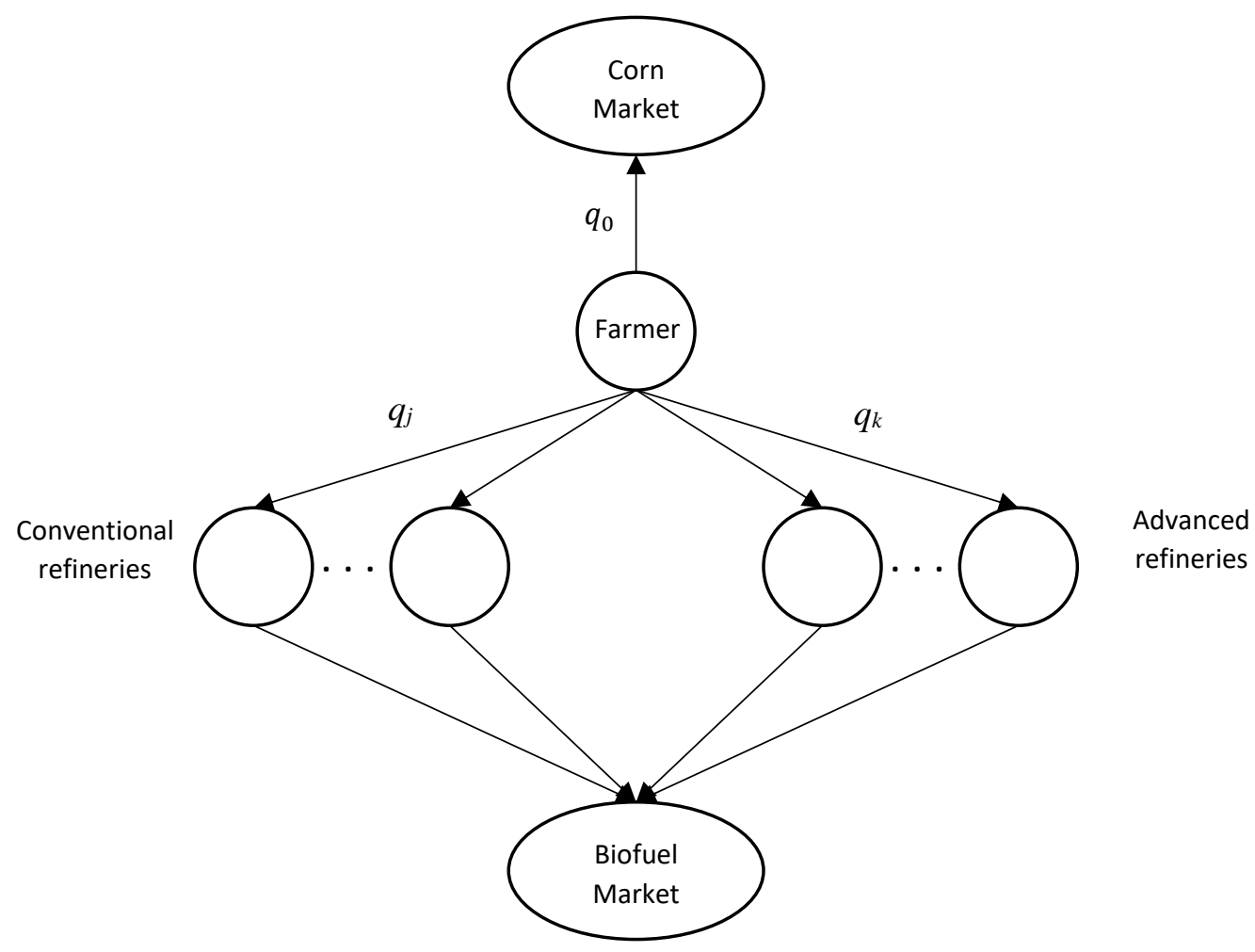

Figure 4.1 Generalized bilevel supply chain network

$x$ : acres of land allocated to corn market

$q_{j}$ : acres of land allocated to corn based refinery $j$

$q_{k}$ : acres of land allocated to energy crop-based refinery $\mathrm{k}$ 
Table 4.1 Notations

\begin{tabular}{|c|c|}
\hline \multicolumn{2}{|l|}{ Sets } \\
\hline $\bar{J}$ & Set of conventional refineries \\
\hline$K$ & Set of advanced refineries \\
\hline$I=J \cup K$ & Set of all refineries \\
\hline \multicolumn{2}{|c|}{ Parameters } \\
\hline $\bar{a}$ & Biofuel market reservation price $(\$ / \mathrm{gl})$ \\
\hline$b$ & Biofuel market price function slope $(\$ / \mathrm{gl})$ \\
\hline$a_{0}$ & Corn market reservation price (\$/acre) \\
\hline$b_{0}$ & Corn market price function slope (\$/acre) \\
\hline$\beta_{i}$ & $\begin{array}{l}\text { Conversion factor from an acre of either corn or energy crop land to gallons of biofuel for } \\
\text { the } i^{\text {th }} \text { refinery (gl/acre) }\end{array}$ \\
\hline$c_{i}^{R}$ & Production cost of the $i^{\text {th }}$ refinery $(\$ / \mathrm{gl})$ \\
\hline $\bar{q}_{i}$ & Maximum capacity of the $i^{\text {th }}$ refinery (acres) \\
\hline $\bar{q}_{0}$ & Maximum demand of the food markets \\
\hline$c_{i}^{1}$ & Transportation cost of the farmer's crop from her land to the $i^{\text {th }}$ refinery ( $\$ /$ acre) \\
\hline$c_{i}^{2}$ & Transportation cost of the produced biofuel from the $i^{t h}$ refinery to the biofuel market $(\$ / \mathrm{gl})$ \\
\hline$T_{i}$ & Subsidy rate paid by the government to the $i^{\text {th }}$ advanced refinery $(\$ / \mathrm{gl})$ \\
\hline$c^{e 1}$ & Land expansion cost coefficient for corn cultivation $(\$ /$ acre $)$ \\
\hline$c^{e 2}$ & Land expansion cost coefficient for energy crop cultivation(\$/acre) \\
\hline$c_{i}^{f}$ & Farmer's production cost corresponding to the crop sold to $i^{\text {th }}$ refinery ( $\$ /$ acre) \\
\hline$W$ & Subsidy rate paid by the government to the farmer to grow energy crop ( $\$ /$ acre) \\
\hline$Q$ & Farmer's total capacity (acre) \\
\hline
\end{tabular}

\section{Decision variables}

\begin{tabular}{ll}
\hline$q_{0}$ & Acres of land used to grow corn for the local food market \\
$q_{i}$ & Acres of land allocated to the $i^{t h}$ refinery \\
$p_{i}$ & Price proposed to the farmer by the $i^{\text {th }}$ refinery for an acre of the farmer's land (\$/acre)
\end{tabular}




\subsubsection{Upper-level problem}

Each refinery $r \in \boldsymbol{I}$, behaving as a single leader, solves the problem below to find his optimal price offer to the farmer. Each refinery maximizes its profit shown in Equation (1) consisting of his revenue, purchasing, transportation, and production costs, and the subsidy paid by the government for advanced biofuel production (if any). In Equation (1), the value of $\beta_{r} q_{r}$ is the number of gallons of biofuel produced by refinery $r \in I$.

$$
\max _{p_{r}} \pi_{r}=\left(a-b \sum_{i \in I} \beta_{i} q_{i}\right) \beta_{r} q_{r}-p_{r} q_{r}-c_{r}^{1} q_{r}-c_{r}^{2} \beta_{r} q_{r}-c_{r}^{R} q_{r}+T_{r} \beta_{r} q_{r}
$$

s.t. $\quad 0 \leq p_{r}$

$$
q_{i \in I} \text { solves lower level problem (4)-(7) }
$$

Constraint (2) ensures the positivity of the refinery's price offer. Constraint (3) implies that the optimal land allocation is determined by the farmer for her own profit maximization problem (4)-(7) presented below.

\subsubsection{Lower-level problem}

The lower level problem (4)-(7) defines the farmer's profit maximization problem. For this lower level problem, the refineries' decision variables $\left(p_{i}\right)$ are treated as given input parameters. For a later use, the dual variables corresponding to the constraints are also shown.

$$
\begin{array}{cc}
\max _{q_{0}, q_{1}, \ldots, q_{I}} \pi_{F}=\sum_{i \in I} p_{i} q_{i}+\left(a_{0}-b_{0} q_{0}\right) q_{0}-c^{e 1}\left(\sum_{j \in J} q_{j}+q_{0}\right)^{2}-c^{e 2}\left(\sum_{k \in K} q_{k}\right)^{2}-\sum_{i \in I} c_{i}^{f} q_{i}-c_{1}^{f} q_{0}+W \sum_{k \in K} q_{k} \\
\text { s.t. } \sum_{i \in I} q_{i}+q_{0} \leq Q \quad(\lambda) & \\
0 \leq q_{i} \leq \bar{q}_{i} \quad\left(\mu_{i}^{1}\right) & \forall i \in I \\
0 \leq q_{0} \leq \bar{q}_{0} \quad\left(\mu^{2}\right) &
\end{array}
$$


The profit of the farmer (Equation (4)) consists of the revenue from selling crops to refineries and the food market, her production costs, and the subsidy given by the government to grow energy crops. The production cost of the farmer consists of quadratic terms (to which we refer as land expansion costs), and linear terms. Second order production cost structure have been used to capture this diseconomy of scale and the increasing marginal cost as the farmland grows. Constraint (5) ensures that the maximum capacity of the farmer is not violated. Constraints (6) and (7) specify the upper limits of refineries' production and corn market capacity.

We need to include the problem (4)-(7) in the refineries' problems according to Equation (3) to transform our bilevel problem to a single level formulation. The equivalent KKT conditions of the lower level problem (4)-(7) are shown below:

$$
\begin{array}{ll}
L=\pi_{F}+\lambda\left(Q-q_{0}-\sum_{i} q_{i}\right)+\sum_{i} \mu_{i}^{1}\left(\bar{q}_{i}-q_{i}\right)+\mu^{2}\left(\bar{q}_{0}-q_{0}\right) & \\
0 \leq \lambda+\mu_{j}^{1}+2 c^{e 1} \sum_{i \in J} q_{i}+c_{j}^{f}-p_{j} \perp q_{j} \geq 0 & \forall j \in J \\
0 \leq \lambda+\mu_{k}^{1}+2 c^{e 2} \sum_{i \in K} q_{i}+c_{k}^{f}-p_{k}-W \perp q_{k} \geq 0 & \\
0 \leq \lambda+\mu^{2}+2 c^{e 1} q_{0}+c_{1}^{f}-a_{0}+2 b_{0} q_{0} \perp q_{0} \geq 0 & \forall k \in K \\
0 \leq Q-\sum_{i \in I} q_{i}-q_{0} \perp \lambda \geq 0 & \\
0 \leq \bar{q}_{i}-q_{i} \perp \mu_{i}^{1} \geq 0 & \\
0 \leq \bar{q}_{0}-q_{0} \perp \mu^{2} \geq 0 &
\end{array}
$$

\subsubsection{Reformulation as an MPEC}

The problem of a refinery $r \in I$ can be written as an MPEC by replacing Equation (3) with Equations (8)(13) in the refinery $r$ 's problem. The resulting MPEC is the optimization problem that each refinery faces 
and solves simultaneously with other refineries. Equations (14)-(32) represent the MPEC formulation for each refinery $r$ along with the Lagrangian multiplier of each constraint shown in parenthesis to be used in the EPEC formulation.

$\max _{p_{r}} \pi_{r}=\left(a-b \sum_{i \in I} \beta_{i} q_{i}\right) \beta_{r} q_{r}-p_{r} q_{r}-c_{r}^{1} q_{r}-c_{r}^{2} \beta_{r} q_{r}-c_{r}^{R} q_{r}+T_{r} \beta_{r} q_{r}$

s.t. $\quad 0 \leq p_{r}$

$\left(\xi_{r}\right)$

$0 \leq q_{i}$

$\left(\varepsilon_{i r}^{1}\right)$

$\forall i \in I$

$0 \leq q_{0}$

$\left(\varepsilon_{r}^{2}\right)$

$0 \leq \mu_{i}^{1}$

$\left(\psi_{i r}^{1}\right)$

$\forall i \in I$

$0 \leq \mu^{2}$

$\left(\psi_{r}^{2}\right)$

$0 \leq \lambda$

$\left(\psi_{r}^{3}\right)$

$0 \leq \bar{q}_{i}-q_{i}$

$\left(\alpha_{i r}^{1}\right)$

$\forall i \in I$

$0 \leq \bar{q}_{0}-q_{0}$

$\left(\alpha_{r}^{2}\right)$

$0 \leq Q-\sum_{i \in I} q_{i}-q_{0}$

$\left(\alpha_{r}^{3}\right)$

$0 \leq \lambda+\mu_{j}^{1}+2 c^{e 1} \sum_{i \in J} q_{i}+c_{j}^{f}-p_{j}$

$\left(\delta_{j r}^{1}\right)$

$\forall j \in J$

$0 \leq \lambda+\mu_{k}^{1}+2 c^{e 2} \sum_{i \in K} q_{i}+c_{k}^{f}-p_{k}-W$

$\left(\delta_{k r}^{1}\right)$

$\forall k \in K$

$0 \leq \lambda+\mu^{2}+2 c^{e 1} q_{0}+c_{1}^{f}-a_{0}+2 b_{0} q_{0}$

$\left(\delta_{r}^{2}\right)$

$q_{j}\left(\lambda+\mu_{j}^{1}+2 c^{e 1} \sum_{i \in J} q_{i}+c_{j}^{f}-p_{j}\right)=0$

$\left(\theta_{j r}^{1}\right)$

$\forall j \in J$

$q_{k}\left(\lambda+\mu_{k}^{1}+2 c^{e 2} \sum_{i \in K} q_{i}+c_{k}^{f}-p_{k}-W\right)=0$

$\left(\theta_{k r}^{1}\right)$

$\forall k \in K$

$q_{0}\left(\lambda+\mu^{2}+2 c^{e 1} q_{0}+c_{1}^{f}-a_{0}+2 b_{0} q_{0}\right)=0$

$\left(\theta_{r}^{2}\right)$

$\lambda\left(Q-\sum_{i \in I} q_{i}-q_{0}\right)=0$

$\left(\varphi_{r}^{3}\right)$ 


$$
\begin{aligned}
& \mu_{i}^{1}\left(\bar{q}_{i}-q_{i}\right)=0 \quad\left(\varphi_{i r}^{1}\right) \quad \forall i \in I \\
& \mu^{2}\left(\bar{q}_{0}-q_{0}\right)=0 \quad\left(\varphi_{r}^{2}\right)
\end{aligned}
$$

\subsubsection{EPEC}

The MPEC problem represented in the previous subsection holds for each refinery $r \in I$. To determine the collective decisions of all refineries and the farmer, we need to aggregate all the MPECs and find the joint solution(s) of them. The joint solution of MPECs is called generalized Nash Equilibrium (GNE). The EPEC formulation is derived by simultaneously consideration of the KKT conditions of all refineries' MPECs. To find the KKT conditions of an MPEC, the derivatives of its Lagrangian function $L_{r}$ are taken with respect to the decision variables, shown in Equations (33)-(39) along with the complementarity conditions, primal feasibility and dual feasibility conditions. Equation (33) corresponds to the derivative with respect to the refineries' price offers, Equations (34)-(36) are derivatives with respect to the decision variables of the farmer and Equations (37)-(39) correspond to the derivatives with respect to the Lagrangian multipliers of the mentioned constraints. Equations (40)-(51) define positivity constraints and the respective complementarity conditions and Equation (52) define the primal feasibility conditions of the MPECs. The EPEC formulation for a numerical example with two refineries of each type has been shown in Appendix B.1.

$$
\begin{aligned}
& L_{r}=\left(a-b \sum_{i \in I} \beta_{i} q_{i}\right) \beta_{r} q_{r}-p_{r} q_{r}-c_{r}^{R} q_{r}+T_{r} \beta_{r} q_{r}-c_{r}^{1} q_{r}-c_{r}^{2} q_{r} \\
& +\sum_{i \in I} \delta_{i r}^{1}\left(\lambda+\mu_{i}^{1}+2 c^{e 1} \sum_{j \in J} q_{j}+c_{i}^{f}-p_{i}-W\right)+\sum_{i \in I} \varepsilon_{i r}^{1} q_{i} \\
& -\sum_{i \in I} \theta_{i r}^{1} q_{i}\left(\lambda+\mu_{i}^{1}+2 c^{e 1} \sum_{j \in I} q_{j}+c_{i}^{f}-p_{i}-W\right) \\
& +\delta_{r}^{2}\left(\lambda+\mu^{2}+2 c^{e 2} q_{0}+c_{1}^{f}-a_{0}+2 b_{0} q_{0}\right)+\varepsilon_{r}^{2} q_{0} \\
& -\theta_{r}^{2} q_{0}\left(\lambda+\mu^{2}+2 c^{e 2} q_{0}+c_{1}^{f}-a_{0}+2 b_{0} q_{0}\right) \\
& +\alpha_{r}^{3}\left(Q-\sum_{i \in I} q_{i}-q_{0}\right)+\psi_{r}^{3} \lambda-\varphi_{r}^{3} \lambda\left(Q-\sum_{i \in I} q_{i}-q_{0}\right) \\
& +\sum_{i \in I} \alpha_{i r}^{1}\left(\bar{q}_{i}-q_{i}\right)+\sum_{i \in I} \psi_{i r}^{1} \mu_{i}^{1}-\sum_{i \in I} \varphi_{i r}^{1} \mu_{i}^{1}\left(\bar{q}_{i}-q_{i}\right)
\end{aligned}
$$




$$
\begin{aligned}
& +\alpha_{r}^{2}\left(\bar{q}_{0}-q_{0}\right)+\psi_{r}^{2} \mu^{2}-\varphi_{r}^{2} \mu^{2}\left(\bar{q}_{0}-q_{0}\right)+\xi_{r} p_{r} \\
& \frac{\partial L_{r}}{\partial p_{r}}=-q_{r}-\delta_{r r}^{1}+\theta_{r}^{1} q_{r}+\xi_{r}=0 \\
& \frac{\partial L_{r}}{\partial q_{r}}=-b \beta_{r}^{2} q_{r}+\beta_{r}\left(a-b \sum_{i \in I} \beta_{i} q_{i}\right)-p_{r}-c_{r}^{R}+T_{r} \beta_{r}-c_{r}^{1}-c_{r}^{2}+\sum_{j \in J} 2 c^{e 1} \delta_{j r}^{1}+\varepsilon_{r r}^{1}-\sum_{\substack{j \in J \\
j \neq r}} 2 c^{e 1} \theta_{j r}^{1} q_{j} \\
& -\theta_{r r}^{1}\left(\lambda+\mu_{r}^{1}+2 c^{e 1} \sum_{j \in J} q_{j}+c_{r}^{f}-p_{r}\right)-2 c^{e 1} \theta_{r r}^{1} q_{r}-\alpha_{r}^{3}+\varphi_{r}^{3} \lambda-\alpha_{r r}^{1}+\varphi_{r}^{2} \mu_{r}^{1}=0 \\
& \frac{\partial L_{r}}{\partial q_{r}}=-b \beta_{r}^{2} q_{r}+\beta_{r}\left(a-b \sum_{i \in I} \beta_{i} q_{i}\right)-p_{r}-c_{r}^{R}+T_{r} \beta_{r}-c_{r}^{1}-c_{r}^{2}+\sum_{k \in K} 2 c^{e 2} \delta_{k r}^{1}+\varepsilon_{r r}^{1}-\sum_{\substack{k \in K \\
k \neq r}} 2 c^{e 2} \theta_{k r}^{1} q_{k} \\
& -\theta_{r r}^{1}\left(\lambda+\mu_{r}^{1}+2 c^{e 2} \sum_{i \in K} q_{i}+c_{r}^{f}-p_{r}-W\right)-2 c^{e 2} \theta_{r r}^{1} q_{r}-\alpha_{r}^{3}+\varphi_{r}^{3} \lambda-\alpha_{r r}^{1}+\varphi_{r}^{2} \mu_{r}^{1}=0 \quad r \in K \\
& \frac{\partial L_{r}}{\partial q_{0}}=2 \delta_{r}^{2} b_{0}+2 c^{e 1} \delta_{r}^{2}+\varepsilon_{r}^{2}-\theta_{r}^{2}\left(\lambda+\mu^{2}+2 c^{e 1} q_{0}+c_{1}^{f}-a_{0}+2 b_{0} q_{0}\right) \\
& -\theta_{r}^{2} q_{0}\left(2 c^{e 1}+2 b_{0}\right)-\alpha_{r}^{3}+\varphi_{r}^{3} \lambda-\alpha_{r}^{2}+\varphi_{r}^{2} \mu^{2}=0 \\
& r \in I \\
& \frac{\partial L_{r}}{\partial \lambda}=\sum_{i \in I} \delta_{i r}^{1}-\sum_{i \in I} \theta_{i r}^{1} q_{i}+\delta_{r}^{2}-\theta_{r}^{2} q_{0}+\psi_{r}^{3}-\varphi_{r}^{3} Q=0 \\
& \frac{\partial L_{r}}{\partial \mu_{i}^{1}}=\delta_{i r}^{1}-\theta_{i r}^{1} q_{i}+\psi_{i r}^{1}-\varphi_{i r}^{1}\left(\bar{q}_{i}-q_{i}\right)=0 \\
& \frac{\partial L_{r}}{\partial \mu^{2}}=\delta_{r}^{2}-\theta_{r}^{2} q_{0}+\psi_{r}^{2}-\varphi_{r}^{2}\left(\bar{q}_{0}-q_{0}\right)=0 \\
& 0 \leq \delta_{i r}^{1} \perp\left(\lambda+\mu_{i}^{1}+2 c^{e 1} \sum_{j \in J} q_{j}+c_{i}^{f}-p_{i}\right) \geq 0 \\
& 0 \leq \delta_{i r}^{1} \perp\left(\lambda+\mu_{i}^{1}+2 c^{e 2} \sum_{k \in K} q_{k}+c_{i}^{f}-p_{i}-W\right) \geq 0 \\
& 0 \leq \delta_{r}^{2} \perp\left(\lambda+\mu^{2}+2 c^{e 2} q_{0}+c_{1}^{f}-a_{0}+2 b_{0} q_{0}\right) \geq 0 \\
& 0 \leq \xi_{r} \perp p_{r} \geq 0 \\
& 0 \leq \varepsilon_{i r}^{1} \perp q_{i} \geq 0 \\
& 0 \leq \varepsilon_{r}^{2} \perp q_{0} \geq 0 \\
& 0 \leq \psi_{i r}^{1} \perp \mu_{i}^{1} \geq 0 \\
& r \in I \\
& r \in J \\
& r \in I \\
& \forall r, i \in I \\
& \forall r \in I \\
& \forall i \in J, r \in I \\
& \forall i \in K, r \in I \\
& \forall r \in I \\
& \forall r \in I \\
& \forall i, r \in I \\
& \forall r \in I \\
& \forall i, r \in I
\end{aligned}
$$


$0 \leq \psi_{r}^{2} \perp \mu^{2} \geq 0$

$\forall r \in I$

$0 \leq \psi_{r}^{3} \perp \lambda \geq 0$

$\forall r \in I$

$0 \leq \alpha_{r}^{3} \perp\left(Q-\sum_{i \in I} q_{i}-q_{0}\right) \geq 0$

$\forall r \in I$

$0 \leq \alpha_{r}^{2} \perp\left(\bar{q}_{0}-q_{0}\right) \geq 0$

$\forall r \in I$

$0 \leq \alpha_{i r}^{1} \perp\left(\bar{q}_{i}-q_{i}\right) \geq 0$

$\forall i, r \in I$

(24)-(32)

\subsection{Solution Methodology}

There are two popular methods to solve an EPEC. Many works are based on diagonalization algorithms. EPECs may have multiple equilibria, but no methodology to identify the exact number of equilibria is known; however, diagonalization algorithms can verify if a given stationary point is an equilibrium (Allevi et al. 2018). Diagonalization uses the definition of the Generalized Nash Equilibrium in identifying an equilibrium, in which none of the players are willing to unilaterally deviate from that solution. Diagonalization algorithms, which use NLP solvers, are conceptually simple and easy to implement, and this has made them a good choice for engineers and applied economists ( $\mathrm{Su} 2005)$.

One of the diagonalization algorithms used to solve EPEC problems is based on the Jacobi diagonalization method introduced by $\mathrm{Hu}$ (2002) and later with a small variation by Su (2005) as described below where $x^{i,(j)}$ is the vector of decision variables for the $i^{\text {th }}$ MPEC and $y^{(j)}$ is the vector of shared decision variables among MPECs. The main drawback of this method is that the global convergence cannot be guaranteed. Another drawback of the mentioned diagonalization methods is that we have no control on the selection of the equilibrium found as the solution in case of multiplicity. For more details and the proof of the local convergence, please see $\mathrm{Su}(2005)$.

Step 1: Choose a starting point $\left(x^{(0)}, y^{(0)}\right)=\left(x^{1,(0)}, \ldots, x^{I,(0)}, y^{(0)}\right)$, the maximum number of iterations $J$ and an accuracy tolerance $\varepsilon>0$. 
Step 2: Given the current iteration point $\left(x^{(j)}, y^{(j)}\right)$ solve the $i^{\text {th }} M P E C$ for each $i=1, \ldots, I$ using the existing NLP solvers while fixing $x^{-i,(j)}$ and store the solution as $x^{i,(j+1)}$

Step 3: If $j<J$, then add one unit to $j$ and go to Step 2, else calculate the accuracy as the second norm of the difference between $x^{i,(j)}$ and $x^{i,(j+1)}$ for $i=1, \ldots, I$ and report the solution if the accuracy is less the tolerance for all the MPECs. Otherwise, report "No solution found".

Because of the mentioned drawbacks, we adopt another approach, which is based on reformulating the EPEC as a mixed integer linear programming (MILP). This approach has been used in several recent works (Wogrin and Barquin, 2013, Allevi et al. 2018).

The MPEC of each refinery is already nonconvex because of the existence of complementarity constraints (Ralph 2007, page 2). Due to the nonconvexity of the MPEC problem, the corresponding KKT conditions provide stationary solutions among which there can be zero, one or multiple equilibria (Allevi et al. 2018, page 722). Although conditions on the existence as well as the possibility of having multiple equilibria in a general equilibrium model have been previously studied (Kehoe 1998), obtaining such conditions for EPECs is not practical (Wogrin and Barquin, 2013). We follow the approach of Ruiz et al. (2012) to linearize the KKT conditions of each MPEC problem and derive the equivalent set of mixed-integer linear (MIL) conditions. The resultant formulation may include multiple equilibria, and we need to select the most beneficial one depending on the decision maker's objective. Considering the existence of subsidies and the role of the government, which tries to improve the welfare of the society, we select an objective function that maximizes the total social welfare of all firms. Hence, our optimization problem can be summarized as:

$$
\begin{aligned}
& \max \pi=\left(a-b \sum_{i \in I} \beta_{i} q_{i}\right)\left(\sum_{i \in I} \beta_{i} q_{i}\right)-\sum_{i \in I}\left(c_{i}^{1}+c_{i}^{2}+c_{i}^{R}\right) q_{i}+\left(a_{0}-b_{0} q_{0}\right) q_{0}-c^{e 1}\left(\sum_{j \in I} q_{j}+x\right)^{2}-c^{e 2}\left(\sum_{k \in K} q_{k}\right)^{2}+\sum_{i \in I} W_{i} q_{i}-\sum_{i \in I} c_{i}^{f} q_{i}-c_{i}^{f} q_{0} \\
& +\sum_{i \in I} T_{i} \beta_{i} q_{i}+b\left(\sum_{i \in I} \beta_{i} q_{i}\right)^{2} / 2+b_{0} q_{0}^{2} / 2
\end{aligned}
$$

s.t. Linear version of EPEC

$\pi$ in (53) consists of the total profit of the farmer, the profit of refineries and the consumer surplus from the corn and fuel markets, a common term to be found in most of EPEC studies. There are two sources of 
nonlinearity in the EPEC (33)-(52): the complementarity conditions, and the quadratic terms from the product of the KKT multipliers and other variables. The complementarities are replaced with their linear equivalent as shown in (54)-(55) for Equation (40). In (54)-(55), $M$ is a large enough constant and $t_{k r}^{\delta 1}$ are binary variables. When $t_{k r}^{\delta 1}$ is zero, constraint (55) becomes inactive and $\delta_{k r}^{1}$ is forced to be zero. The complementarity conditions and their linearization are shown in the Appendix B.2.

$$
\begin{array}{lc}
\delta_{i r}^{1} \leq M t_{i r}^{\delta 1} & i \in J, r \in I \\
\lambda+\mu_{i}^{1}+2 c^{e 2} \sum_{j \in J} q_{j}+c_{i}^{f}-p_{i}-W_{i} \leq M\left(1-t_{i r}^{\delta 1}\right) & i \in J, r \in I
\end{array}
$$

For the quadratic terms in (33)-(39), we follow Pereira et al. (2005) and Wogrin and Barquin, (2013) and apply binary expansion on the KKT multipliers $\theta_{i i}^{1}, \theta_{i}^{2}, \varphi_{i i}^{1}, \varphi_{i}^{2}, \varphi_{i}^{3}$ that make some of the terms quadratic. The basic idea of binary expansion is to approximate a continuous variable by a set of discrete values, which are in the form of $2^{f}$, where $f=0, \ldots, n$ and $n$ is a non-negative integer. For a better understanding, the binary expansion of $\theta_{i j}^{1}, i, j \in I$ is shown below as an example.

$\theta_{i j}^{1}=\Delta_{\theta 1} \sum_{f=0}^{n} 2^{f} b_{f i j}^{\theta 1}$

In (56), $\Delta_{\theta 1}$ determines the incremental value of $\theta_{i j}^{1}$, and $b_{f i j}^{\theta 1}$ are binary variables. Having defined the binary expansion of the KKT multipliers, we can linearize their product with a given variable (e.g. $\left.q_{r}\right)$ by introducing a new set of variables which are defined as the multiplication of the binary variables and the given variable. For example, the quadratic term $\theta_{i j}^{1} q_{i}$, can be replaced by $\theta_{i j}^{1} q_{i}=\Delta_{\theta 1} \sum_{f=0}^{n} 2^{f} b_{f i j}^{\theta 1} q_{i}=\Delta_{\theta 1} \sum_{f=0}^{n} 2^{f} z_{f i j}^{\theta 1, q}$

where, $z_{f i j}^{\theta 1, q}$ stands for $b_{f i j}^{\theta 1} q_{i}$ and is defined by the following constraints:

$0 \leq z_{f i j}^{\theta 1, q} \leq M b_{f i j}^{\theta 1}$

$0 \leq q_{i}-z_{f i j}^{\theta 1, q} \leq M\left(1-b_{f i j}^{\theta 1}\right)$ 
According to (57)-(58), when $b_{f i j}^{\theta 1}$ is one, $z_{f i j}^{\theta 1, q}$ takes a non-negative value bounded by $M$, which is a suitably large constant and when $b_{f i j}^{\theta 1}$ is zero, $z_{f i j}^{\theta 1, q}$ would be zero as well. The linearization of all nonlinear equations constituting the EPEC are shown in the Appendix B.2.

\subsection{Numerical example and estimation comparison}

In this section we solve an example, which is similar to what was considered by Yu and Miller (2013) to find the amount of subsidies needed to produce sufficient amount of advanced biofuel and study the socioeconomic impacts of the government's subsidy. This analysis is done for two types of the farmer's production cost structures (constant and increasing marginal cost) in subsection 4.4.1. We also check the effect of the number of refineries on the selected performance measures in subsection 4.4.2. A sensitivity analysis is done on important parameters and market conditions in subsection 4.4.3.

The example consists of a farmer, two identical corn-based refineries, two identical energy crop-based refineries $(I=\{1, \ldots, 4\})$, and food and fuel markets (Figure 4.2). The size of this example is the same as the one in Yue and Miller (2013) for a meaningful comparison. The parameter values for this example are realistic and from the literature, given in Table 4.2. We assume the refineries have zero transportation costs to concentrate on the effect of subsidies regardless of transportation costs.

We consider the values of the subsidy paid to the farmer $(W)$ and the advanced refineries $(T)$ in a reasonable range, (e.g., $[0,2]$ for $T$ and $[0,2000]$ for $W$ ) and obtain the results including the total amount of incentives under a given policy and check if it can meet the RFS's goal, which requires the ratio $(\rho)$ of the conventional biofuel production over the advanced biofuel production to be at least $\rho=0.71 .(=15 \mathrm{Mg} / 21 \mathrm{Mg})\left(110^{\text {th }}\right.$ U.S. Congress 2007). If $\rho<0.71$ the supply chain is underperforming; if $\rho>0.71$ it is over performing. 
Table 4.2 Parameters

\begin{tabular}{llll}
\hline $\begin{array}{l}\text { Parameter } \\
\text { value }\end{array}$ & Source & $\begin{array}{l}\text { Parameter } \\
\text { value }\end{array}$ & Source \\
\hline$a=10(\$)$ & Bai et al., (2016) & $c_{1}^{R}=c_{2}^{R}=890(\$ / \mathrm{ha})$ & Luo and Miller (2013) \\
$b=0.0005\left(\frac{\Delta p}{\Delta q}\right)$ & Bai et al., (2016) & $c_{3}^{R}=c_{4}^{R}=1,211(\$ / \mathrm{ha})$ & Luo and Miller (2013) \\
$a_{0}=1500(\$)$ & Alizamir et al., (2015) & $c^{e 1}=c^{e 2}=50(\$ / \mathrm{ha})$ & Luo and Miller (2013) \\
$b_{0}=0.0005\left(\frac{\Delta p}{\Delta q}\right)$ & Alizamir et al., (2015) & $c_{1}^{f}=c_{2}^{f}=711(\$ / \mathrm{ha})$ & Luo and Miller (2013) \\
$\beta_{1}=\beta_{2}=1,009(\mathrm{~g} / \mathrm{ha})$ & Luo and Miller (2013) & $c_{3}^{f}=c_{4}^{f}=760(\$ / \mathrm{ha})$ & Luo and Miller (2013) \\
$\beta_{3}=\beta_{4}=1,489(\mathrm{~g} / \mathrm{ha})$ & Luo and Miller (2013) & $Q=30 \mathrm{M}(\mathrm{ha})$ & Farming land \\
$\bar{q}_{0}=0.7 Q$ & & & \\
\hline
\end{tabular}

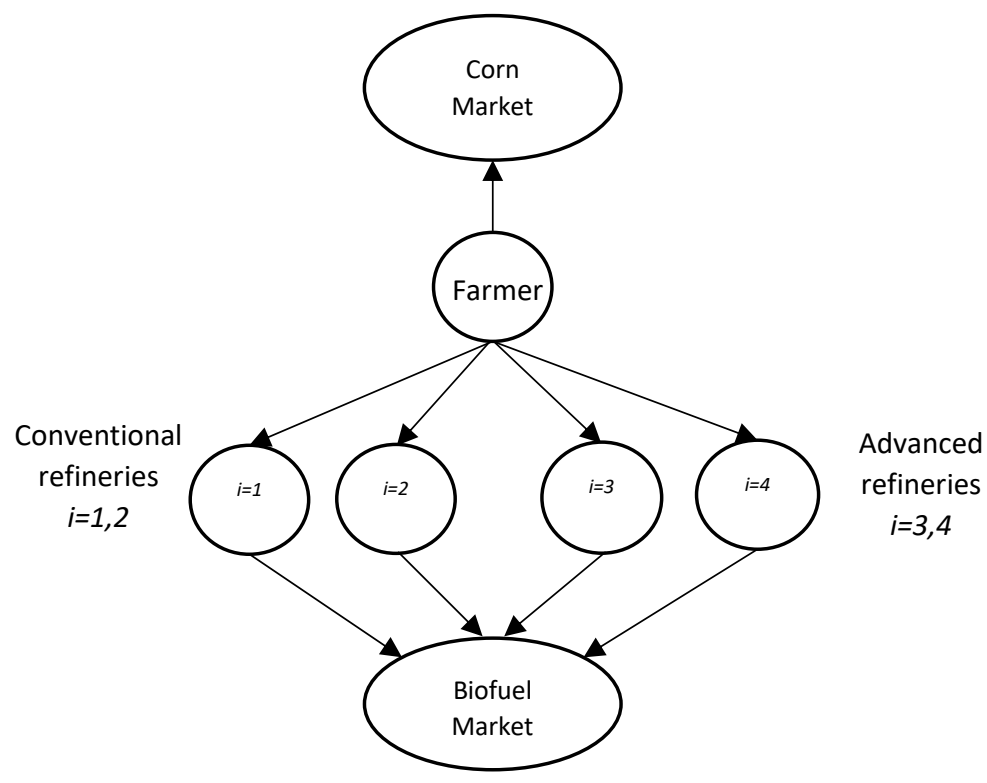

Figure 4.2 Representation of the example supply chain

\subsubsection{Effect of the farmer's cost structure}

For the two types of cost structures (constant and increasing marginal cost), we randomly select 4 subsidy policies among those with $\rho \approx 0.71$ and calculate the value of the performance measures including the subsidy expenditures (farmer subsidy and producer subsidy), and social welfare change (total producer surplus increase and total consumer surplus increase), shown in Figure 4.3. Farmer subsidy and producer 
subsidy represent the total subsidy paid to the farmer and the advanced refineries respectively. Total producer surplus increase and total consumer surplus increase are defined as the respective surplus change from the case without subsidy.

Looking at the sum of the farmer subsidy and the producer subsidy (the left bar of each pair in Figure 4.3) for the two cost structures, we observe that $\$ 35 \mathrm{~B}$ and $\$ 40 \mathrm{~B}$ are the required, respectively. In a similar analysis by Luo and Miller (2013), they show that under an average between the conservative and optimistic technological settings, an approximate of $\$ 25 \mathrm{~B}$ is required to meet the RFS. They use a linear cost structure for the farmer. The difference between their results and ours (with the linear cost structure) could be from not considering the food market and other simplifying assumptions such as constant biofuel market price.

The effect of the farmer's cost structure on the producer and consumer's surplus is also shown in Figure 4.3. Looking at the total consumer surplus increase reveals that consumers are better off under the constant marginal cost. With constant marginal cost, which requires less total cost than the case with increasing marginal cost in our example, farmers are willing to grow more corn for the food market and this lowers the corn price, while the increasing marginal cost structure drives them to provide more land for the biofuel industry, which leads to higher corn price in the food market. However, the refineries are better off in the latter case with larger total producer surplus increase.

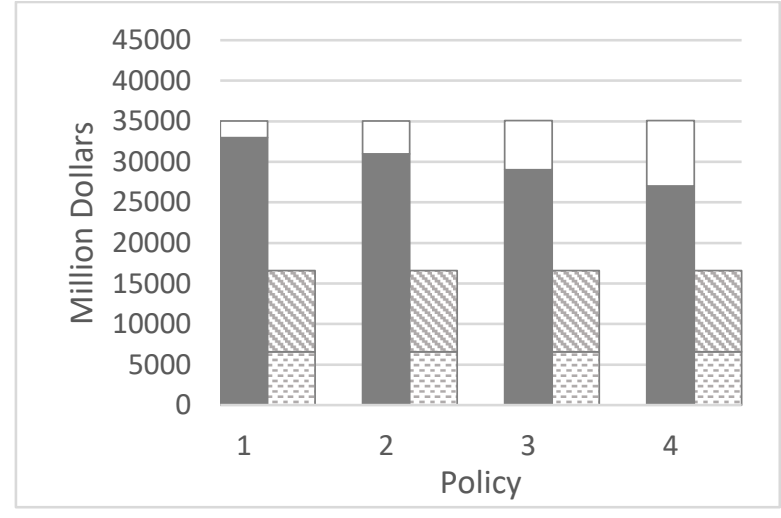

(a) Constant marginal cost

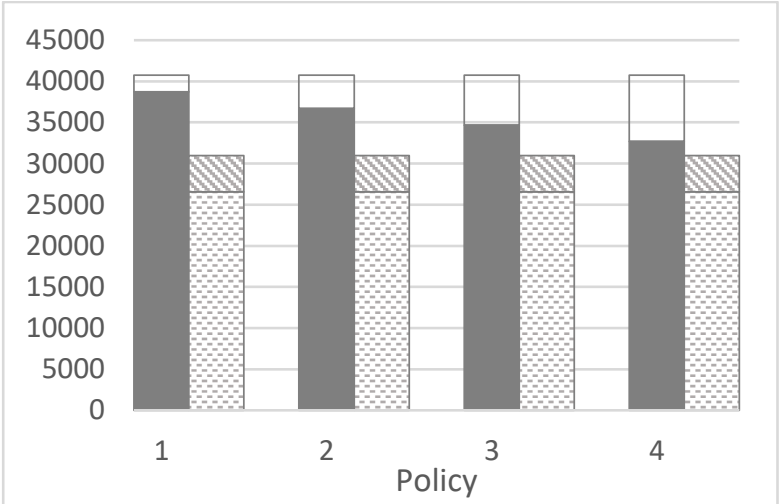

(b) Increasing marginal cost

Figure 4.3 Subsidy and social welfare comparison.

Farmer subsidy ( $($ ). Producer subsidy $(\square)$. Total producer surplus increase (E). Total consumer surplus increase $(\mathbb{D})$. 
Another observation from Figure 4.3 is that the government spends the same amount of expenditure (farmer subsidy plus producer subsidy) under all the policies with $\rho \approx 0.71$. The way a certain amount of budget is distributed between the farmer and the advanced refineries does not make a difference in the value of $\rho$. In addition, the same amount of the budget under different distributions between the farmer and producers results in the same values of total consumer surplus increase and total producer surplus increase. It is worth mentioning that these findings hold for both types of the cost structures. The same conclusion is drawn by Bajgiran et al. (2018), in which they consider the same biofuel supply chain with two refineries and a single farmer.

It is worth mentioning that the government's subsidy is larger than the increase in the social welfare increase under either of the cost structures (Figure 3). Bajgiran et al. (2018), using a numerical example, show that, with constant marginal cost, if the farmer allocates the same amount of land to the corn and energy crop refineries, the subsidy offset is possible to happen. In this example, none of the equilibria suggests an equal land allocation between the two types of refineries and the subsidy offset is not seen for either of the cost structures. Figure 3 shows that the change of the marginal cost from constant to increasing results in higher social welfare with a higher cost of the government subsidy. The increase of the social welfare is because of the higher profit of the refineries with the increased subsidy payment.

\subsubsection{Effect of competition}

In this subsection, we study the effect of the number of refineries on the supply chain's performance. To make the comparison, we solve the example for two cases: (1) with a single refinery of each type and (2) with two identical refineries of each type. In Figure 4.4a, farmer subsidy and producer subsidy are shown for the two cases. We observe that as the number of refineries increases, the amount of subsidy required to meet the RFS mandate also increases. Because of the higher competition, the offered prices for land allocation from both types of refineries are higher, and advanced refineries, while being less efficient, should be compensated by more subsidy. This decline of the advanced refineries' competing power is the 
major source of increase in the total subsidy. In Figure 4.4b, we observe a slight increase in the total social welfare because of the higher competition. As the competition gets stronger, the refineries increase their price offer, and this leads to lower profit for them. However, that decrease is compensated by the increase in the consumer surplus in the fuel market and increase in the total social welfare.

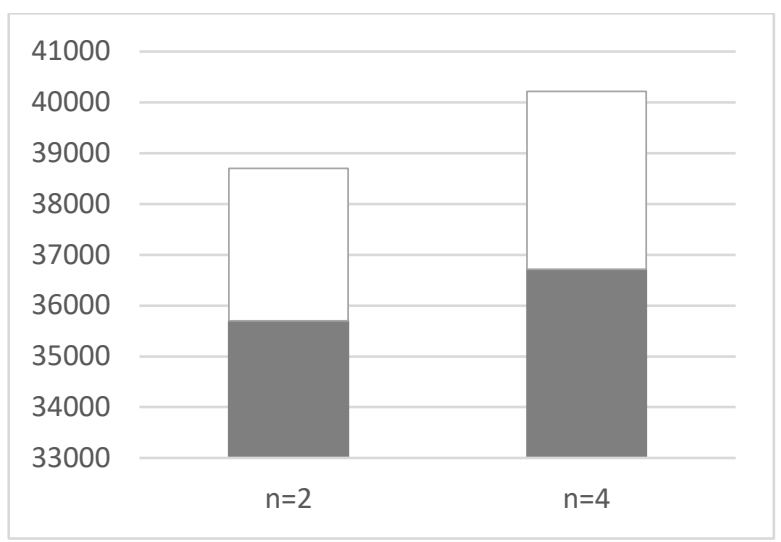

(a) Subsidy

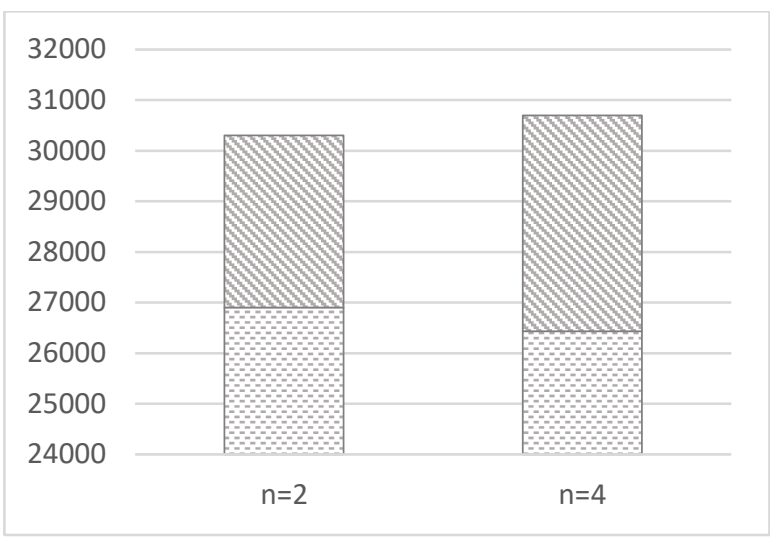

(a) Social welfare

Figure 4.4 Effect of competition on the performance measures.

Farmer subsidy ( ). Producer subsidy $(\square)$. Total producer surplus increase $\left(\square_{--}^{-}\right)$. Total consumer surplus increase $(\mathbb{N})$.

\subsubsection{Sensitivity Analysis}

A sensitivity analysis is made using a numerical example to study the effect of technological advancements and economic conditions. We assume a $\pm 10 \%$ change from the baseline value in four parameters including the conversion factor of energy crop to biofuel $\left(\beta_{2}\right)$, production cost of advanced biofuel $\left(c_{2}^{R}\right)$, the slope of the corn market price functions (b), and the biofuel market price function (b0), illustrated in each panel of Figure 4.5, respectively.

In Figure 4.5a and 4.c, we change the parameters related to the efficiency of the advanced biofuel production, i.e. conversion factor and production cost. Figure $4.5 \mathrm{a}$ shows a negative relationship between the conversion factor and the subsidy required to meet the RFS mandate. When refineries can produce more advanced biofuel from the same amount of energy crop (higher conversion factor), they become more competitive against conventional refineries and need less support to stay in the market. The producer 
subsidy in Figure 4.5a shows this trend. The conversion factor has a negative effect on the farmer subsidy, which can be justified by the higher energy crop price offers receive; making them more willing to grow energy crops with lower incentives from the government. The change of the subsidy payment would have a direct impact on their share from the social welfare, e.g., a decrease in the subsidy paid to refineries results in a decrease in the producer surplus. The opposite trends hold for the increase of the production cost parameter as shown in Figure 4.5c, with lower impact though.

In Figure 4.5(b, d), we observe the effect of the food and fuel market elasticities on the performance measures. The decreasing subsidy trend in Figure $4.5 \mathrm{~b}$ tells that as the food market becomes more elastic, less subsidy is required to meet the RFS mandate. The reason is that the increase of the corn market elasticity results in lower corn quantity in the market and lower farmer's revenue from selling to the corn market while her cost decreases faster because of the expansion costs (increasing marginal cost). Hence, the balance is positive in favor of the farmer, and she would need less subsidy. The biofuel market elasticity has a minimal increasing effect on the subsidy requirement since the higher elasticity of the biofuel market would result in lower revenue of the refineries, which needs to be made up with more subsidy payment. However, effect of the biofuel market elasticity on other measures is negligible. Finally, we observe the effect the quadratic terms' coefficients (in the farmer's cost function) on the studied measures. It is shown that as the coefficients increase, the farmer subsidy would also increase, but there is a minimal decrease in the producers' subsidy. The social welfare would also slightly decrease as a result of that change, which mostly is due to the lower corn production.

The sensitivity of considered measures are different. The resultant change from the deviation of the conversion factor $\left(\beta_{2}\right)$ is the most significant followed by the corn market price slope $(b)$, advanced biofuel production $\operatorname{cost}\left(c_{2}^{R}\right)$ and biofuel market price slope $\left(b_{0}\right)$. As an example, a $10 \%$ change in the conversion factor results in almost $20 \%$ change in the subsidy payment while a $10 \%$ change in the production cost results in less than $3 \%$ change in the subsidy payment. 


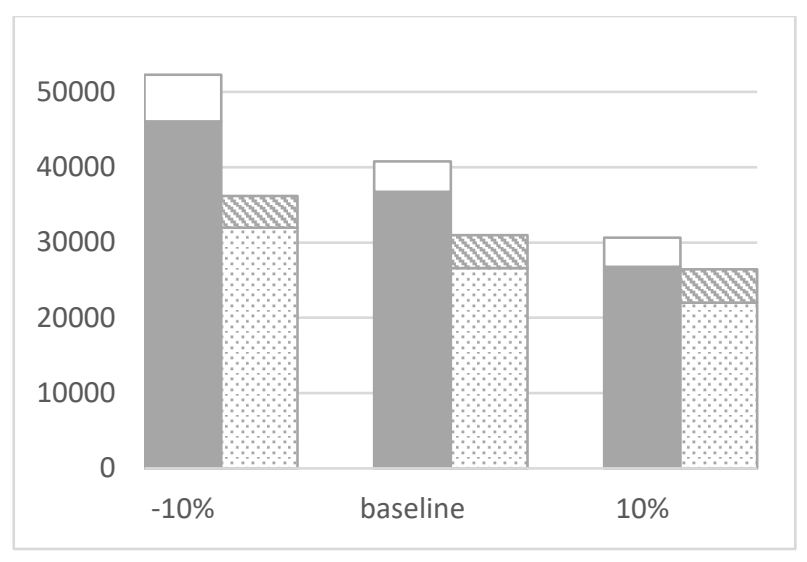

(a) $\beta_{2}$

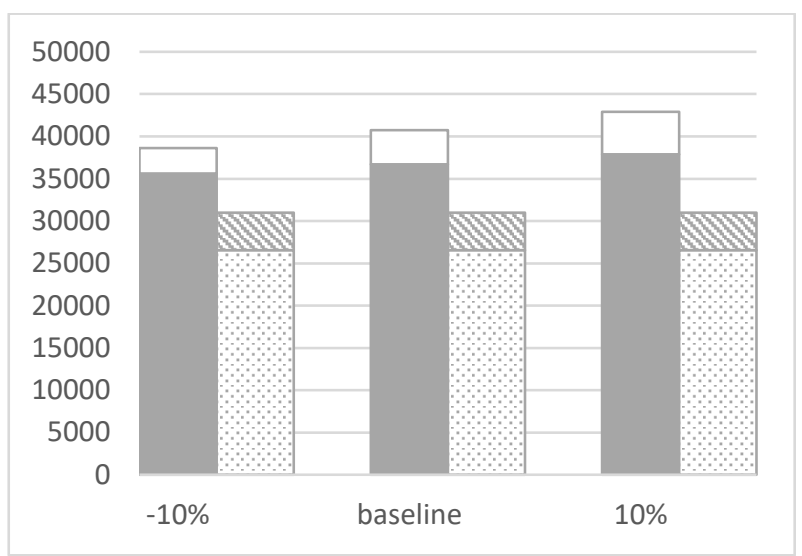

(c) $c_{2}^{R}$

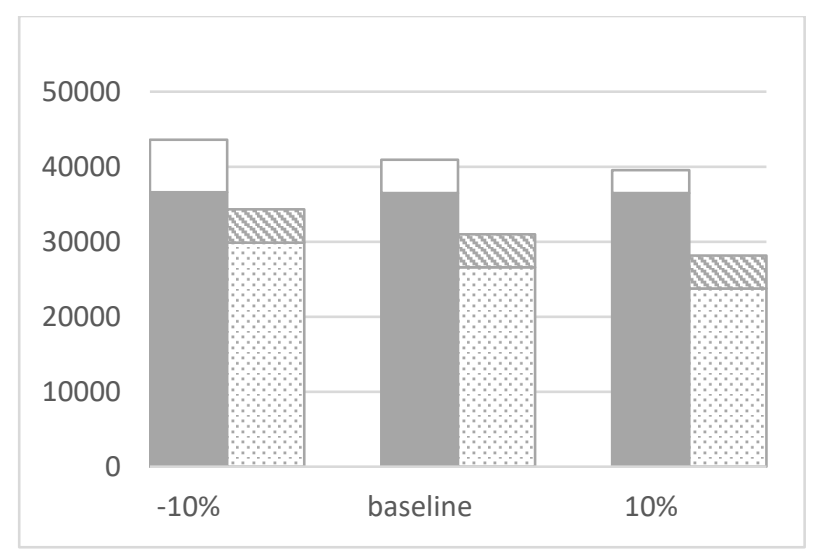

(b) $b$

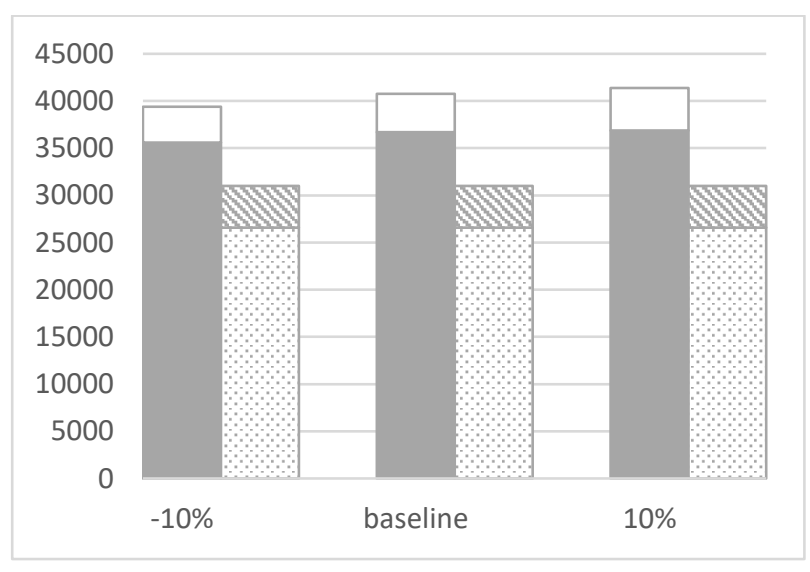

(d) $b_{0}$

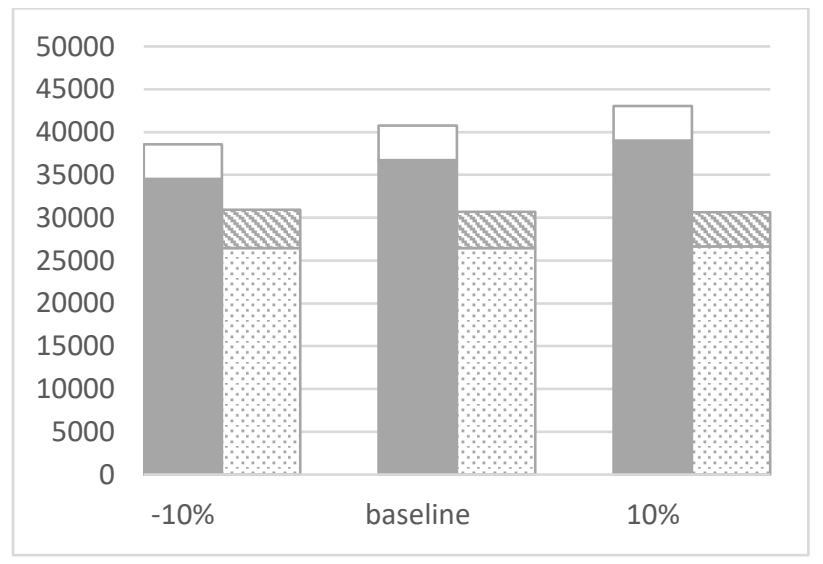

(e) $c_{i}^{e}$

Figure 4.5 Sensitivity analysis on critical parameters.

Farmer subsidy ( $\square$. Producer subsidy $(\square)$. Total producer surplus increase ( $\left.{ }_{--}^{--}\right)$. Total consumer surplus increase $(\mathbb{N})$. 


\subsection{Conclusion}

In this chapter, we address a biofuel supply chain problem to study the subsidy policies and incentives, paid directly to participating firms. Subsidies are studied as they are one of the few options that government can use to motivate the advanced biofuel refineries and farmers (who can grow energy crops) to invest more in this industry and meet the RFS mandate which requires the production of $36 \mathrm{~B}$ gallons of biofuel by 2022 , out of which 21B gallons should be advanced/cellulosic biofuel. To address the problem, a non-linear game theory-based optimization model is designed, which incorporates real world complications such as transportation, diseconomies of scale, capacity restrictions and several producers. Our model is one of the first among those who utilize EPEC formulation in the biofuel supply chain modeling.

We use a numerical example to study the derived equilibrium and perform several analyses on it. First, we find that based on our model, which is more comprehensive than other existing works, more subsidy is required to meet the RFS mandate than what is claimed before. Second, having considered several refineries, we verify and numerically generalize our findings from our previous work (Bajgiran et al. 2018) and study the effect of the number of refineries on the performance of the supply chain. We find that as the number of refineries increases, the subsidy requirement and the total social welfare increase as well. We also verify that it is unlikely to payback the government's expenditure on subsidy by the increase of the social welfare even when there is no farmer's land expansion cost. Third, we perform a sensitivity analysis to study the effect of the more important parameters on the performance of the supply chain and conclude that the advanced biofuel technology improvement can incredibly reduce the amount of subsidy requirement and needs more attention from the scientific point of view.

Although many realistic conditions are consolidated in our model, there are other opportunities to make it even closer to real world practice. A future direction for this research is considering a nonlinear inverse demand function for the food and fuel markets. This is something that has been widely neglected in the literature and needs to be addressed based on industry practitioners' idea. Another future research direction would be addressing the uncertainty that exists in the agriculture industry and is inevitable. It is apparent 
that models with the ability to consider the effect of unpredictable factors such weather on the farmer's production quantity would be more reliable. 


\section{Chapter 5}

\section{Summary of findings}

\subsection{Introduction}

We present various sets of models in Chapters 2-4. A summary of models in shown in Figure 5.1. In Chapter 2, we consider four cases based on the existence of the food market, and the const structure of the farmer. In this chapter, it is assumed that the refineries have no capacity limit and can process all the farmer's land. One refinery is considered for each type. In Chapter 3, we relax the unlimited capacity of the refineries and make the problem more realistic but maintain the four cases already discussed. In Chapter 4, we generalize the problem by considering several refineries of each type and remove the cases where there was no food market.
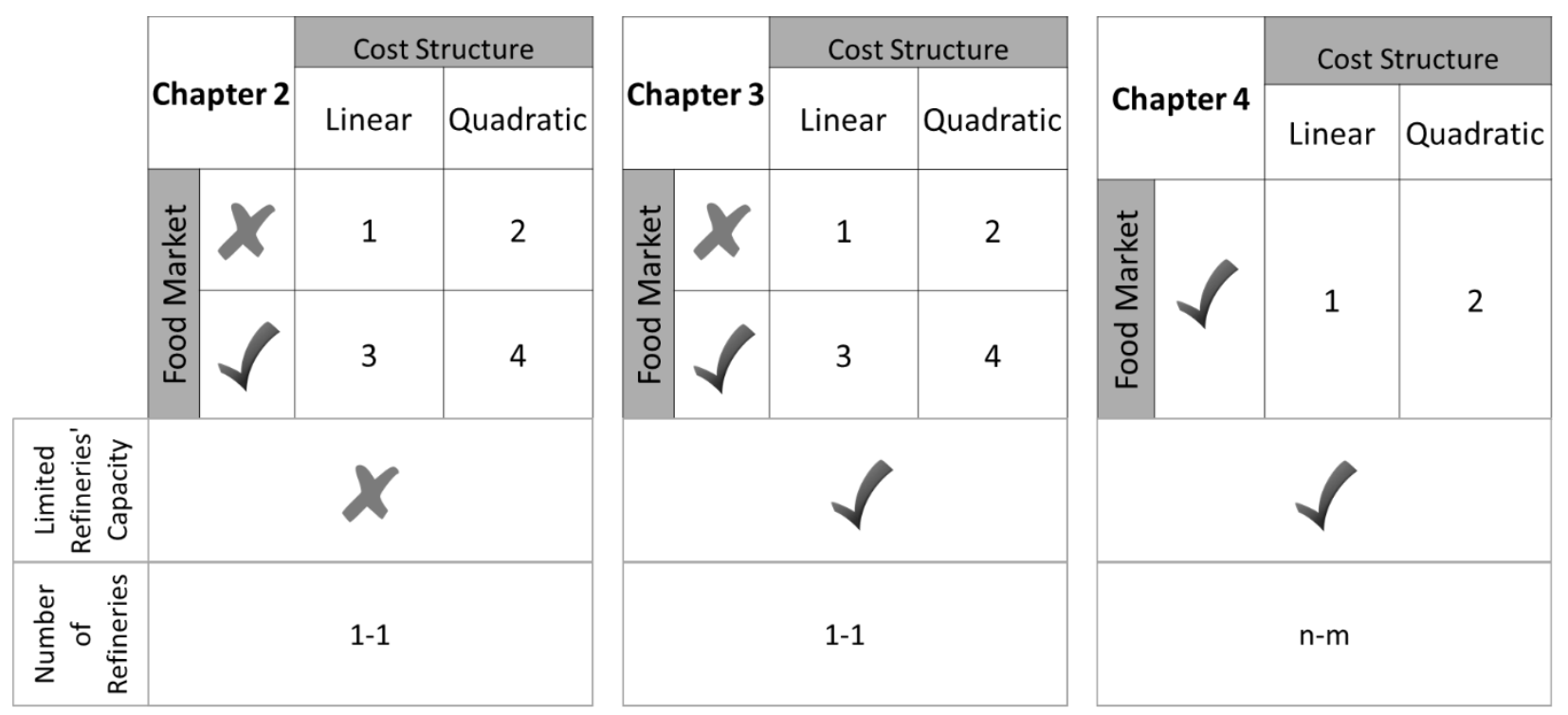

Figure 5.1 Summary of considered models in all chapters 

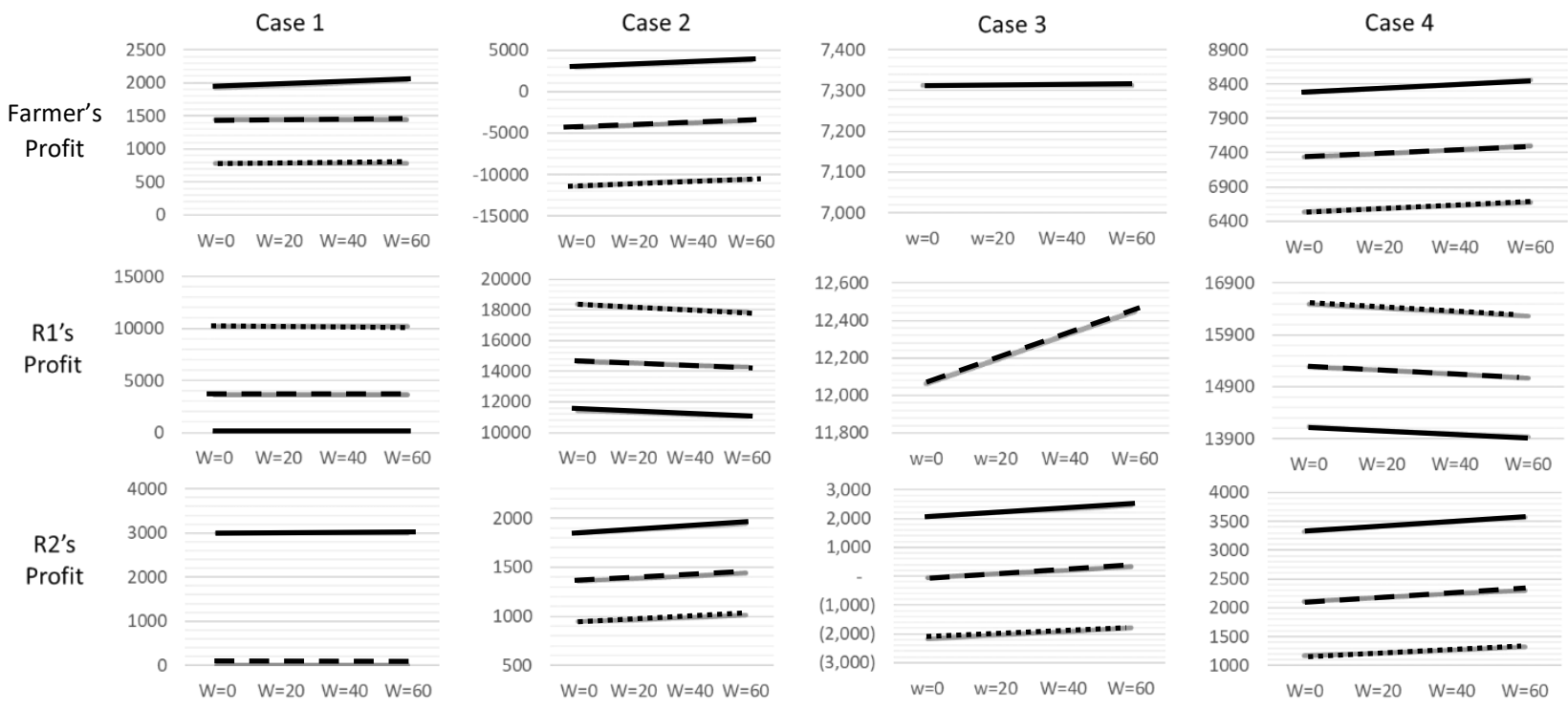

Figure 5.2 Effect of the subsidies on the profits

The effect of the subsidies on the profit of the firms remains the same through all the chapters. The effects are shown in Figure 5.2. On average, the farmer is better off in Cases 3 and 4 where there is food market and subsidies make her better off in all cases, unless case 3. The reason is the domain that we have selected in which the farmer would share the land equally among the refineries regardless of the subsidies. R1 is on average better off at Case 2 (no food market and increasing marginal cost) followed by Case 4 (food market and constant marginal cost) than the other two cases, showing that R1 can get advantage from the absence of food market and the increasing marginal cost of the farmer. R2 benefits from subsidies in all cases and It is in Case 4 (food market and increasing marginal cost) (followed by Case 2) where R2 has the highest average profit, showing that the increasing marginal cost of the farmer can be beneficial for R2 as well.

\subsection{Effect of the capacity constraint (Chapter 3)}

In Chapter 3, we add refineries' capacity constraint to the problem to make it more realistic. The first effect of the capacity constraint that we address is the number of the domains of the offered prices. As shown in Figure 5.3, the number of domains will increase in all cases, except Case 1 where it would remain the same. The increase is because of the fact that refineries have limited capacity and even if they lose the competition, 
they have the chance to get some land, and this increases the possible domains in the solution space. Second, we are interested to study the effect of the capacity constraints on the socio-economic measures. As discussed in Chapters 2-3, we selected the most representative domain for each case to perform our analysis and do not observe any change between the results of Chapters 2 and 3 in those selected domains, except in Case 1. As shown in Figure 5.4, because of the capacity limit, the refinery which loses the competition would have the chance to get some land. For this reason, under the capacitated model, refineries would offer lower prices, because they know they would be given some land even of they lose. This results in higher refineries' profit and lower farmer's profit.

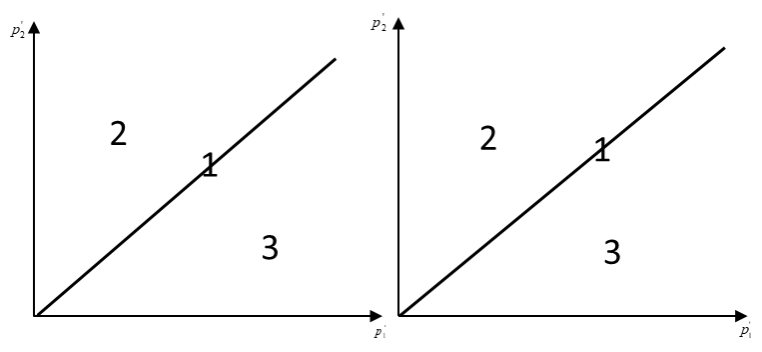

Case 1 (No food market-linear cost)

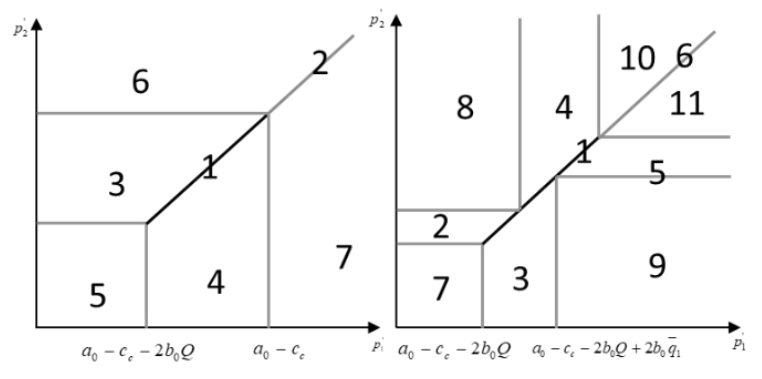

Case 3 (Food market-linear cost)

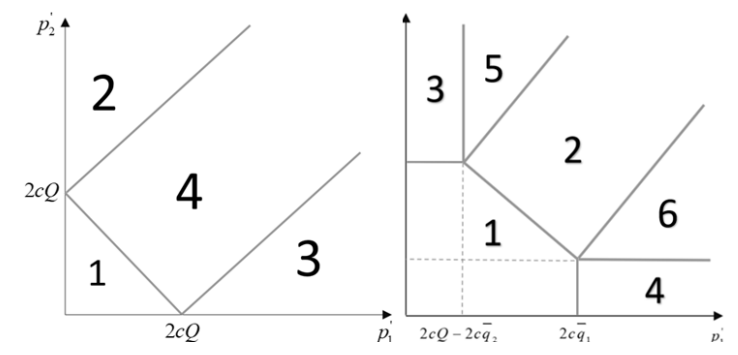

Case 2 (No food market-nonlinear cost)

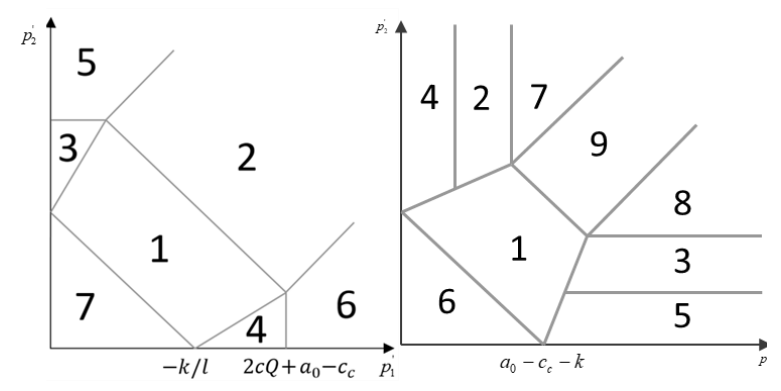

Case 4 (Food market-nonlinear cost)

Figure 5.3 Comparison of the domains of price offers 


\begin{tabular}{ccc}
\hline Capacitated & & Uncapacitated \\
\hline$p_{i}^{\max }$ & $<$ & $p_{i}^{\max }$ \\
$\pi_{\text {refineries }}$ & $>$ & $\pi_{\text {refineries }}$ \\
$\pi_{\text {Farmer }}<$ & $\pi_{\text {Farmer }}$ \\
\hline
\end{tabular}

Figure 5.4 Comparison of results for Case 1 after adding capacity limit

\subsection{Effect of competition (Chapter 4)}

Next, we are interested in the effect of the number of refineries (competition) on the socio-economic measures. Looking at the subsidy payments in Figure 5.5, we find that as more refineries are built, more subsidy is required for both refineries and the farmer. Also, we find that the increase of refineries would benefit the consumers by increasing the consumer surplus. The reason is that the competition between refineries would result in lower biofuel prices in the market, and although the we observe a shrinkage in the producer surplus, the total social welfare would increase as more refineries are built.

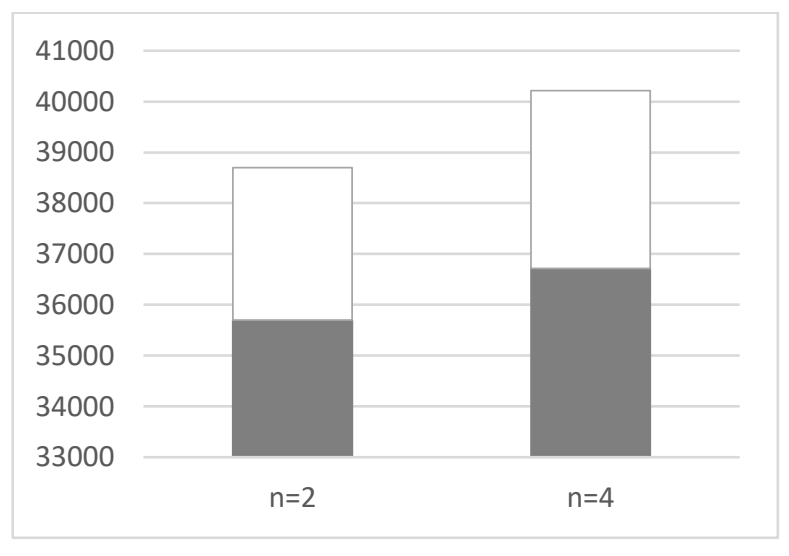

(a) Subsidy

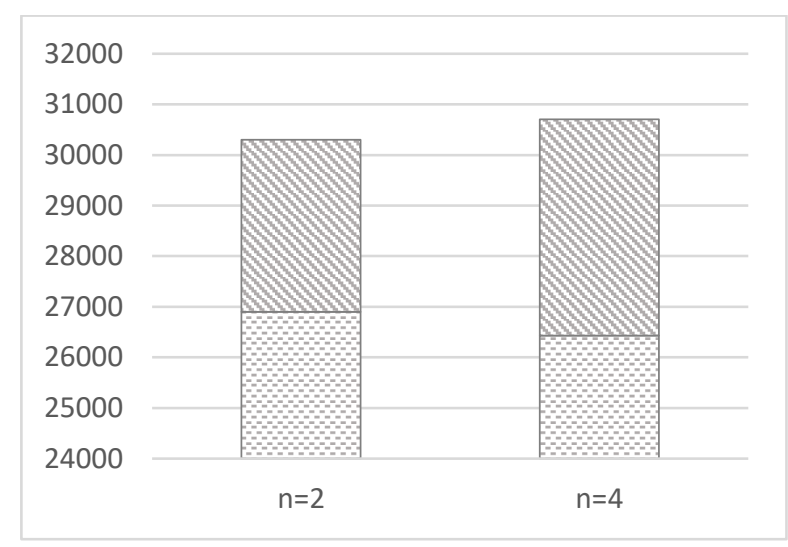

(a) Social welfare

Figure 5.5 Effect of the refineries' competition

Farmer subsidy ( $($ ). Producer subsidy $(\square)$. Total producer surplus increase ( increase $(\mathbb{N})$. 


\section{References}

110th U.S. Congress, 2007. Energy Independence and Security Act of 2007, H.R.6.

Annual Energy Outlook: https://www.eia.gov/outlooks/aeo/pdf/0383(2016).pdf

Agbo, M., Rousselière, D., \& Salanié, J. (2015). Agricultural marketing cooperatives with direct selling: A cooperative-noncooperative game. Journal of Economic Behavior \& Organization, 109, 56-71.

Akgul, O., Shah, N., \& Papageorgiou, L. G. (2012). Economic optimisation of a UK advanced biofuel supply chain. Biomass and Bioenergy, 41, 57-72.

Alizamir, S., Iravani, F., \& Mamani, H. (2015). Price vs. revenue protection: An analysis of government subsidies in the agriculture industry. Working paper.

Allevi, E., Conejo, A. J., Oggioni, G., Riccardi, R., \& Ruiz, C. (2018). Evaluating the strategic behavior of cement producers: An equilibrium problem with equilibrium constraints. European Journal of Operational Research, 264(2), 717-731.

An, H., Wilhelm, W. E., \& Searcy, S. W. (2011). A mathematical model to design a lignocellulosic biofuel supply chain system with a case study based on a region in Central Texas. Bioresource technology, 102(17), 7860-7870.

Awudu, I., \& Zhang, J. (2013). Stochastic production planning for a biofuel supply chain under demand and price uncertainties. Applied Energy, 103, 189-196.

Awudu, I., \& Zhang, J. (2012). Uncertainties and sustainability concepts in biofuel supply chain management: A review. Renewable and Sustainable Energy Reviews, 16(2), 1359-1368.

Bai, Y., Hwang, T., Kang, S., \& Ouyang, Y. (2011). Biofuel refinery location and supply chain planning under traffic congestion. Transportation Research Part B: Methodological, 45(1), 162-175.

Bai, Y., Ouyang, Y., \& Pang, J. S. (2012). Biofuel supply chain design under competitive agricultural land use and feedstock market equilibrium. Energy Economics, 34(5), 1623-1633.

Bai, Y., Ouyang, Y., \& Pang, J. S. (2016). Enhanced models and improved solution for competitive biofuel supply chain design under land use constraints. European Journal of Operational Research, 249(1), 281-297.

Bajgiran, H. A., Jang, J., Fang, X., Peoples H. J., (2018). A biofuel supply chain equilibrium analysis with subsidy consideration. Submitted to International Journal of Energy Research.

Cobuloglu, H. I., \& Büyüktahtakın, İ. E. (2015). Food vs. biofuel: An optimization approach to the spatio-temporal analysis of land-use competition and environmental impacts. Applied Energy, 140, 418-434.

Camanzi, L., Malorgio, G., \& Azcárate, T. G. (2011). The role of producer organizations in supply concentration and marketing: a comparison between European countries in the fruit and vegetable sector. Journal of Food Products Marketing, 17(2-3), 327-354.

Chen, X., Huang, H., Khanna, M., \& Önal, H. (2011). Meeting the mandate for biofuels: implications for land use, food, and fuel prices. In The intended and unintended effects of US agricultural and biotechnology policies (pp. 223-267). University of Chicago Press.

Dal-Mas, M., Giarola, S., Zamboni, A., \& Bezzo, F. (2011). Strategic design and investment capacity planning of the ethanol supply chain under price uncertainty. Biomass and Bioenergy, 35(5), 2059-2071.

Debertin, David L., "Agricultural Production Economics" (2012). Agricultural Economics Textbook Gallery. 1. http://uknowledge.uky.edu/agecon_textbooks/1

Ferris, M. C., \& Munson, T. S. (2000). Complementarity Problems in GAMS and the PATH Solver1. Journal of Economic Dynamics and Control, 24(2), 165-188.

Ferris, J. J., \& Joshi, S. (2010). Prospects for Ethanol and Biodiesel, 2008 to 2017 and Impacts on Agriculture and Food. In Handbook of bioenergy economics and policy (pp. 91-111). Springer New York. Gerbens-Leenes P.W., Hoekstra A.Y., Van der Meer Th. H., 2009a. The Water footprint of bioenergy and other primary energy carriers. Ecol. Econ. 68 (4), $1052-1060$. 
Gabriel, S. A., Conejo, A. J., Fuller, J. D., Hobbs, B. F., \& Ruiz, C. (2012). Complementarity modeling in energy markets, ser. International series in operations research \& management science.

Giarola, S., Zamboni, A., \& Bezzo, F. (2011). Spatially explicit multi-objective optimisation for design and planning of hybrid first and second generation biorefineries. Computers \& Chemical Engineering, 35(9), 1782-1797.

$\mathrm{Hu}$, X., \& Ralph, D. (2007). Using EPECs to model bilevel games in restructured electricity markets with locational prices. Operations research, 55(5), 809-827.

Hu, X. (2002): Mathematical Programs with Complementarity Constraints and Game Theory Models in Electricity Markets, Ph.D. Thesis, Department of Mathematics and Statistics, University of Melbourne.

Huang, H., Khanna, M., Önal, H., \& Chen, X. (2013). Stacking low carbon policies on the renewable fuels standard: Economic and greenhouse gas implications. Energy Policy, 56, 5-15.

Huang, Y., Fan, Y,. Chen, C,. 2014, An Integrated Biofuel Supply Chain to Cope with Feedstock Seasonality and Uncertainty. Transportation Science 48(4):540-554.

Huang Y., Chen Y., 2014, Analysis of an imperfectly competitive cellulosic biofuel supply chain, Journal of Transportation Research Part E

Jang, W., \& Klein, C. M. (2011). Supply chain models for small agricultural enterprises. Annals of Operations Research, 190(1), 359-374.

Kazempour, S. J., Conejo, A. J., \& Ruiz, C. (2013). Generation investment equilibria with strategic producers-Part I: Formulation. IEEE Transactions on Power Systems, 28(3), 2613-2622.

Kehoe, Timothy J. 1998. Uniqueness and Stability. In Elements of General Equilibrium Analysis, ed. Alan Kirman, 38-87. Malden, MA: Blackwell.

Koh, A. (2012). An evolutionary algorithm based on Nash dominance for equilibrium problems with equilibrium constraints. Applied soft computing, 12(1), 161-173.

Leyffer, S., \& Munson, T. (2010). Solving multi-leader-common-follower games. Optimisation Methods \& Software, 25(4), 601623.

Li, Y., Tseng, C.L. and Hu, G., 2015. Is now a good time for Iowa to invest in cellulosic biofuels? A real options approach considering construction lead times. International Journal of Production Economics, 167, pp.97-107.

Luo, Z. Q., Pang, J. S., \& Ralph, D. (1996). Mathematical programs with equilibrium constraints. Cambridge University Press.

Luo, Y., Miller S., 2013. A game theory analysis of market incentives for US switchgrass ethanol. Ecological Economics, 93 (2013) $42-56$.

Marufuzzaman M., Eksioglu S. D., Hernandez R., 2014, Environmentally Friendly Supply Chain Planning and Design for Biodiesel Production via Wastewater Sludge, Journal of Transportation Science

Nasiri, F., \& Zaccour, G. (2009). An exploratory game-theoretic analysis of biomass electricity generation supply chain. Energy Policy, 37(11), 4514-4522.

Nuñez, H. M., Önal, H., \& Khanna, M. (2013). Land use and economic effects of alternative biofuel policies in Brazil and the United States. Agricultural Economics, 44(4-5), 487-499.

Palak, G., Ekşioğlu, S.D. and Geunes, J., 2014. Analyzing the impacts of carbon regulatory mechanisms on supplier and mode selection decisions: An application to a biofuel supply chain. International Journal of Production Economics, 154, pp.198-216.

Papapostolou C., Kondili E., Kaldellis John K., 2011, Development and implementation of an optimization model for biofuel supply chain, Journal of ENERGY. 
Panichelli L., Gnansounou E., 2008, GIS-based approach for defining bioenergy facilities' location: A case study in Northern Spain based on marginal delivery costs and resources competition between facilities, Biomass and Bioenergy.

Parikh, A. (1979). Estimation of supply functions for coffee. Applied Economics, 11(1), 43-54.

Pereira, M. V., Granville, S., Fampa, M. H., Dix, R., \& Barroso, L. A. (2005). Strategic bidding under uncertainty: a binary expansion approach. IEEE Transactions on Power Systems, 20(1), 180-188.

Peterson, W. L. (1997). Are large farms more efficient? (No. 13411). University of Minnesota, Department of Applied Economics.

Pozo, D., \& Contreras, J. (2011). Finding multiple nash equilibria in pool-based markets: A stochastic EPEC approach. IEEE Transactions on Power Systems, 26(3), 1744-1752.

Renewable Fuel Standard Program: https://www.epa.gov/renewable-fuel-standard-program/program-overview-renewable-fuelstandard-program

Ralph, D. (2007). Nonlinear programming advances in mathematical programming with complementarity constraints. Royal Society.

RFS 2014-2016: https://www.epa.gov/renewable-fuel-standard-program/final-renewable-fuel-standards-2014-2015-and-2016and-biomass-based

Ruiz, C., Conejo, A. J., \& Smeers, Y. (2012). Equilibria in an oligopolistic electricity pool with stepwise offer curves. IEEE Transactions on Power Systems, 27(2), 752-761.

Schmidt, J., Leduc, S., Dotzauer, E., Kindermann, G., \& Schmid, E. (2010). Cost-effective CO2 emission reduction through heat, power and biofuel production from woody biomass: A spatially explicit comparison of conversion technologies. Applied Energy, 87(7), 2128-2141.

Su, C. L. (2005). Equilibrium problems with equilibrium constraints: Stationarities, algorithms, and applications. Stanford University.

Tchami, G. (2007). Handbook on Cooperatives for use by Workers' Organizations. Geneva: International Labour Office.

Wu, J., Langpap, C., 2015, The Price and Welfare Effects of Biofuel Mandates and Subsidies, Environ. Resource Econ. 62:35-57.

Wanga X., Ouyang Y., Yang H., Bai Y., 2013, Optimal biofuel supply chain design under consumption mandates with renewable identification numbers, Journal of Transportation Research Part B.

Wickens, M. R., \& Greenfield, J. N. (1973). The econometrics of agricultural supply: an application to the world coffee market. The Review of Economics and Statistics, 433-440.

Wu, J., \& Langpap, C. (2015). The price and welfare effects of biofuel mandates and subsidies. Environmental and Resource Economics, 62(1), 35-57.

Xie, F., Huang a, Y., Eksioglu, S., 2014, Integrating multimodal transport into cellulosic biofuel supply chain design under feedstock seasonality with a case study based on California, Bioresource Technology 152:15-23.

Yue, D., You, F., \& Snyder, S. W. (2014). Biomass-to-bioenergy and biofuel supply chain optimization: overview, key issues and challenges. Computers \& Chemical Engineering, 66, 36-56.

Yue, D., \& You, F. (2014). Game-theoretic modeling and optimization of multi-echelon supply chain design and operation under Stackelberg game and market equilibrium. Computers \& Chemical Engineering, 71, 347-361.

Yue, D., \& You, F. (2014). Fair profit allocation in supply chain optimization with transfer price and revenue sharing: MINLP model and algorithm for cellulosic biofuel supply chains. AIChE Journal, 60(9), 3211-3229.

Wang, M., Han, J., Dunn, J. B., Cai, H., \& Elgowainy, A. (2012). Well-to-wheels energy use and greenhouse gas emissions of ethanol from corn, sugarcane and cellulosic biomass for US use. Environmental research letters, 7(4), 045905. 
Wogrin, S., Barquín, J., \& Centeno, E. (2013). Capacity expansion equilibria in liberalized electricity markets: an EPEC approach. IEEE Transactions on Power Systems, 28(2), 1531-1539.

Zamarripa, M. A., Aguirre, A. M., Méndez, C. A., \& Espuña, A. (2013). Mathematical programming and game theory optimizationbased tool for supply chain planning in cooperative/competitive environments. Chemical Engineering Research and Design, 91(8), 1588-1600.

Zamboni A., Shah N., Bezzo F., 2009, Spatially Explicit Static Model for the Strategic Design of Future Bioethanol Production Systems. 1. Cost Minimization, Energy Fuels, 23 (10), pp 5121-5133

Zamboni A., Bezzo F., Shah N., 2009, Spatially Explicit Static Model for the Strategic Design of Future Bioethanol Production Systems. 2. Multi-Objective Environmental Optimization, Energy Fuels, 23, 5134-5143

Zhang F., Johnson Dana M., Sutherland John W., 2011, A GIS-based method for identifying the optimal location for a facility to convert forest biomass to biofuel, biomass and bioenergy 


\section{Appendices}

\section{Appendix A.1}

$$
\pi_{R 1}=\left(a-b\left(\beta_{1} q_{1}+\beta_{2} q_{2}\right)\right) \beta_{1} q_{1}-p_{1} q_{1}-c_{R 1} q_{1}
$$

From the assumption that refineries will not offer prices lower than the marginal production cost of the farmer, it is clear that $q_{2}=Q-q_{1}$ in the optimal solution of the farmer. We plug this in the profit function of $\mathrm{R} 1$ and get the following:

$\pi_{R 1}=a \beta_{1} q_{1}-b \beta_{1}^{2} q_{1}^{2}-b \beta_{1} \beta_{2} q_{1} Q+b \beta_{1} \beta_{2} q_{1}^{2}-p_{1} q_{1}-c_{R 1} q_{1}$

First we replace all $q_{1}$ 's with $Q$ for the case that R1 wins the Bertrand game and set $\pi_{R 1}=0$ (i.e. R1 quotes its highest possible price such that it wins all the capacity and its profit would be zero). The solution is the maximum price of $\mathrm{R} 1$ to win the Bertrand competition:

$p_{1}^{\max }=a \beta_{1}-b \beta_{1}^{2} Q-c_{R 1}$

Similarly, replacing $q_{1}=Q-q_{2}$ in $\pi_{R 2}$ gives $\pi_{R 2}=a \beta_{2} q_{2}-b \beta_{1} \beta_{2} q_{2} Q+b \beta_{1} \beta_{2} q_{2}^{2}-b \beta_{2}^{2} q_{2}^{2}-p_{2} q_{2}-c_{R 2} q_{2}+T \beta_{2} q_{2}$, and following the same approach, we find the maximum price of $\mathrm{R} 2$ to win the Bertrand competition:

$p_{2}^{\max }=a \beta_{2}-b \beta_{2}^{2} Q-c_{R 2}+T \beta_{2}$

Having found the refineries' maximum prices, we find the maximum marginal profit of the farmer from each crop using those prices and make comparisons. The refinery which provides higher marginal profit for the farmer wins by offering a small value above the maximum price of the rival and wins the land. Tqhis has been shown mathematically in Proposition 2.1.

\section{Appendix A.2}

In domain 1, we have the following farmer's BR from Table 2.3:

$$
\begin{aligned}
q_{1}^{*} & =\frac{p_{1}-c_{c}}{2 c} \\
q_{2}^{*} & =\frac{p_{2}-c_{e}+W}{2 c}
\end{aligned}
$$


Backward induction requires the replacement of these farmer's BR into the refineries' problems shown in Equations (1)-(2) of Chapter 2, and taking the FOC of these equations with respect to their corresponding decision variable, which would give the following:

$$
\begin{aligned}
& p_{1}^{*}=\frac{z e-y f}{x e-y^{2}} \\
& p_{2}^{*}=\frac{z y-x f}{y^{2}-x e}
\end{aligned}
$$

The values of constants in this NE are as follows:

$x=\frac{2 b \beta_{1}^{2}}{2 c}+2$

$y=\frac{b \beta_{1} \beta_{2}}{2 c}$

$z=a \beta_{1}+\frac{2 b \beta_{1}^{2} c_{c}}{2 c}+\frac{b \beta_{1} \beta_{2} c_{e}}{2 c}-\frac{b \beta_{1} \beta_{2} W}{2 c}-c_{R 1}+c_{c}$

$e=\frac{2 b \beta_{2}^{2}}{2 c}+2$

$f=a \beta_{2}+\frac{2 b \beta_{2}^{2} c_{e}}{2 c}+\frac{b \beta_{1} \beta_{2} c_{c}}{2 c}-\frac{2 b \beta_{2}^{2} W}{2 c}-c_{R 2}+c_{e}-W+T \beta_{2}$

In domain 2, we would have the farmer's BR and KKT multipliers from Table 2.3. From the stationary conditions of the KKT conditions, we find the value of $q_{i}^{*}$ as shown below. Given the domain 2 in which $q_{2}$ wins all the land, we set $q_{1}^{*}$ and $q_{2}^{*}$ equal to 0 and Q respectively. Following the positivity conditions on the Lagrangian multipliers would give us the domain limits as shown below:

$$
\begin{aligned}
& q_{1}^{*}=\frac{p_{1}^{\prime}-\lambda_{3}+\lambda_{1}}{2 c}=0 \rightarrow \frac{p_{1}^{\prime}-p_{2}^{\prime}+2 c Q+\lambda_{1}}{2 c}=0 \rightarrow \lambda_{1}=p_{2}^{\prime}-p_{1}^{\prime}-2 c Q>0 \\
& q_{2}^{*}=\frac{p_{2}^{\prime}-\lambda_{3}}{2 c}=Q \rightarrow \lambda_{3}=p_{2}^{\prime}-2 c Q>0
\end{aligned}
$$

Following the backward induction and taking FOC of refineries' problems (Equations (1)-(2) of Chapter 2), with respect to their corresponding decision variable, we find the NE as shown in Proposition 2.2. Similarly, we can find the NE in domain 3. 


\section{Appendix A.3}

In domain 4, having the BR of the farmer from Table 2.3, we follow the backward induction and solve the FOC of both refineries' profit functions simultaneously to obtain the NE shown in Proposition 2.3. The values of constants in Proposition 2.3 are shown below. All the signs are based on the assumption that the conversion rate of corn is higher than that of energy crop $\left(\beta_{1}>\beta_{2}\right)$. Given that assumption, the signs are easy to conclude.

$$
\begin{aligned}
& x=\frac{b \beta_{1} \beta_{2}-b \beta_{1}^{2}}{8 c^{2}}-\frac{1}{2 c}<0 \\
& y=\frac{b \beta_{1}^{2}-b \beta_{1} \beta_{2}}{8 c^{2}}+\frac{1}{4 c}>0 \\
& z=\frac{1}{4 c}\left(-a \beta_{1}+b \beta_{1}^{2} Q+2 c Q+c_{c}+c_{R 1}\right) \\
& d=\frac{b \beta_{2}^{2}-b \beta_{1} \beta_{2}}{8 c^{2}}+\frac{1}{4 c} \\
& e=\frac{b \beta_{1} \beta_{2}-b \beta_{2}^{2}}{8 c^{2}}-\frac{1}{2 c} \\
& f=\frac{1}{4 c}\left(-a \beta_{2}+b \beta_{2}^{2} Q+2 c Q+c_{e}+c_{R 2}-W-T \beta_{2}\right)
\end{aligned}
$$

\section{Appendix A.4}

All the signs are based on the assumption that the conversion rate of corn is higher than that of energy crop $\left(\beta_{1}>\beta_{2}\right)$. Given that assumption, the signs are easy to conclude.

$$
\begin{aligned}
& \frac{\partial p_{1}}{\partial W}=\left(\frac{-y}{x e-y d}\right) \frac{\partial f}{\partial W}=\left(\frac{-y}{x e-y d}\right) \times \frac{-1}{4 c}=\frac{\left(b \beta_{1} \beta_{2}-b \beta_{1}^{2}-2 c\right)<0}{\left[2 b c\left(\beta_{1}^{2}+\beta_{2}^{2}-2 \beta_{1} \beta_{2}\right)\right]>0} \times \frac{-1}{4 c} \rightarrow \frac{\partial p_{1}}{\partial W}>0 \\
& \frac{\partial p_{1}}{\partial T}=\left(\frac{-y}{x e-y d}\right) \frac{\partial f}{\partial T}=\left(\frac{-y}{x e-y d}\right) \times \frac{-\beta_{2}}{4 c}=\frac{\left(b \beta_{1} \beta_{2}-b \beta_{1}^{2}-2 c\right)<0}{\left[2 b c\left(\beta_{1}^{2}+\beta_{2}^{2}-2 \beta_{1} \beta_{2}\right)\right]>0} \times \frac{-\beta_{2}}{4 c} \rightarrow \frac{\partial p_{1}}{\partial T}>0 \\
& \frac{\partial p_{2}}{\partial W}=\left(\frac{-x}{y d-x e}\right) \frac{\partial f}{\partial W}=\left(\frac{-x}{y d-x e}\right) \times \frac{-1}{4 c}-1=\frac{\left(b \beta_{1} \beta_{2}-b \beta_{1}^{2}-4 c\right)<0}{\left[2 b c\left(2 \beta_{1} \beta_{2}-\beta_{1}^{2}-\beta_{2}^{2}\right)-12 c^{2}\right]<0} \times \frac{1}{4 c}-1 \rightarrow \frac{\partial p_{2}}{\partial W}<0 \\
& \frac{\partial p_{2}}{\partial T}=\left(\frac{-x}{y d-x e}\right) \frac{\partial f}{\partial T}=\left(\frac{-x}{y d-x e}\right) \times \frac{-\beta_{2}}{4 c}=\frac{\left(b \beta_{1} \beta_{2}-b \beta_{1}^{2}-4 c\right)<0}{\left[2 b c\left(2 \beta_{1} \beta_{2}-\beta_{1}^{2}-\beta_{2}^{2}\right)-12 c^{2}\right]<0} \times \frac{\beta_{2}}{4 c} \rightarrow \frac{\partial p_{2}}{\partial T}>0
\end{aligned}
$$




$$
\begin{aligned}
& \frac{\partial q_{1}}{\partial W}=\frac{1}{2 c}\left(\frac{\partial P_{1}}{\partial W}-\frac{\partial \lambda_{3}}{\partial W}\right)=\frac{1}{16 c^{2}}\left[\frac{\left(b \beta_{1} \beta_{2}-b \beta_{1}^{2}-2 c\right)}{2 b c\left(2 \beta_{1} \beta_{2}-\beta_{1}^{2}-\beta_{2}^{2}\right)}-\frac{\left(b \beta_{1} \beta_{2}-b \beta_{1}^{2}-4 c\right)}{2 b C\left(2 \beta_{1} \beta_{2}-\beta_{1}^{2}-\beta_{2}^{2}\right)-12 c^{2}}\right] \rightarrow \frac{\partial q_{1}}{\partial W}<0 \\
& q_{1}+q_{2}=Q \rightarrow \frac{\partial q_{2}}{\partial W}=-\frac{\partial q_{1}}{\partial W}>0 \\
& \frac{\partial q_{1}}{\partial T}=\frac{1}{2 c}\left(\frac{\partial P_{1}}{\partial T}-\frac{\partial \lambda_{3}}{\partial T}\right)=\frac{\beta_{2}}{16 c^{2}}\left[\frac{b \beta_{1} \beta_{2}-b \beta_{1}^{2}-2 c}{2 b c\left(2 \beta_{1} \beta_{2}-\beta_{1}^{2}-\beta_{2}^{2}\right)}-\frac{\left(b \beta_{1} \beta_{2}-b \beta_{1}^{2}-4 c\right)}{2 b c\left(2 \beta_{1} \beta_{2}-\beta_{1}^{2}-\beta_{2}^{2}\right)-12 c^{2}}\right] \rightarrow \frac{\partial q_{1}}{\partial T}<0 \\
& q_{1}+q_{2}=Q \rightarrow \frac{\partial q_{2}}{\partial T}=-\frac{\partial q_{1}}{\partial T}>0
\end{aligned}
$$

\section{Appendix A.5}

The values of constants in Proposition 2.5 are as follows:

$$
\begin{aligned}
& k_{1}=\frac{\left(a \beta_{1}-c_{c}-c_{R 1}\right)\left(C+P_{0}^{\prime \prime}\right)}{2 c\left(c+2 P_{0}^{\prime \prime}\right)} \\
& k_{2}=\frac{\left(b \beta_{1}^{2}\right)\left(c+P_{0}^{\prime \prime}\right)}{2 c\left(c+2 P_{0}^{\prime \prime}\right)} \\
& k_{3}=\frac{\left(b \beta_{1} \beta_{2}\right)\left(c+P_{0}^{\prime \prime}\right)}{2 c\left(c+2 P_{0}^{\prime \prime}\right)}=r_{3} \\
& k_{4}=\frac{\left(b \beta_{1}^{2}\right)\left(c+P_{0}^{\prime \prime}\right)-P_{0}^{\prime \prime} b \beta_{1} \beta_{2}}{2 c\left(c+2 P_{0}^{n}\right)} \\
& k_{5}=\frac{c+P_{0}^{\prime \prime}}{2 c\left(c+2 P_{0}^{\prime \prime}\right)} \\
& k_{6}=\frac{P_{0}^{\prime} c-2 Q P_{0}^{\prime \prime} c}{c+2 P_{0}^{\prime \prime}} \\
& k_{7}=\frac{P_{0}^{\prime \prime}}{c+2 P_{0}^{\prime \prime}} \\
& r_{1}=\frac{\left(a \beta_{2}-c_{e}-c_{R 2}+W+T \beta_{2}\right)\left(c+P_{0}^{n}\right)}{2 c\left(c+2 P_{0}^{\prime \prime}\right)} \\
& r_{2}=\frac{\left(b \beta_{2}^{2}\right)\left(c+P_{0}^{\prime \prime}\right)}{2 c\left(c+2 P_{0}^{n}\right)} \\
& r_{3}=\frac{\left(b \beta_{1} \beta_{2}\right)\left(c+P_{0}^{\prime \prime}\right)}{2 c\left(c+2 P_{0}^{\prime \prime}\right)} \\
& r_{4}=\frac{\left(b \beta_{2}^{2}\right)\left(c+P_{0}^{\prime \prime}\right)-P_{0}^{\prime \prime} b \beta_{1} \beta_{2}}{2 c\left(c+2 P_{0}^{\prime}\right)} \\
& r_{5}=\frac{c+P_{0}^{\prime \prime}}{2 c\left(c+2 P_{0}^{\prime \prime}\right)}=k_{5}
\end{aligned}
$$




\section{Appendix B.1}

In this appendix, we show the complete formulation of Equations (1)-(51) of Chapter 4, for an example with four refineries and a farmer and using the realistic parameter values in Table 4.2.

Upper level problem of four refineries in the example:

$$
\begin{aligned}
& \max _{p_{1}} \pi_{1}=\left(10-0.0005\left(693 q_{1}+693 q_{2}+330 q_{3}+330 q_{4}\right)\right) 693 q_{1}-p_{1} q_{1}-890 q_{1} \\
& \max _{p_{2}} \pi_{2}=\left(10-0.0005\left(693 q_{1}+693 q_{2}+330 q_{3}+330 q_{4}\right)\right) 693 q_{2}-p_{2} q_{2}-890 q_{2} \\
& \max _{p_{3}} \pi_{3}=\left(10-0.0005\left(693 q_{1}+693 q_{2}+330 q_{3}+330 q_{4}\right)\right) 330 q_{3}-p_{3} q_{3}-330 q_{3}+330 T q_{3} \\
& \max _{p_{4}} \pi_{4}=\left(10-0.0005\left(693 q_{1}+693 q_{2}+330 q_{3}+330 q_{4}\right)\right) 330 q_{4}-p_{4} q_{4}-330 q_{4}+330 T q_{4} \\
& 0 \leq p_{1} \\
& 0 \leq p_{2} \\
& 0 \leq p_{3} \\
& 0 \leq p_{4}
\end{aligned}
$$

Lower level problem:

$$
\begin{array}{ll}
\max _{x, q_{1}, q_{2}, q_{3}, q_{4}} \pi=\sum_{i=1}^{4} p_{i} q_{i}+\left(1500-0.0005 q_{0}\right) q_{0}-50\left(\sum_{i=1}^{2} q_{i}+q_{0}\right)^{2}-50\left(\sum_{i=3}^{4} q_{i}\right)^{2} \\
-610 q_{1}-610 q_{2}-550 q_{3}-550 q_{4}-610 q_{0}+W\left(q_{3}+q_{4}\right) \\
\sum_{i=1}^{4} q_{i}+q_{0} \leq Q \\
0 \leq q_{1} \leq \bar{q}_{1} & \left(\mu_{1}^{1}\right) \\
0 \leq q_{2} \leq \bar{q}_{2} & \left(\mu_{2}^{1}\right) \\
0 \leq q_{3} \leq \bar{q}_{3} & \left(\mu_{3}^{1}\right) \\
0 \leq q_{4} \leq \bar{q}_{4} & \left(\mu_{4}^{1}\right) \\
0 \leq q_{0} \leq \bar{q}_{0} & \left(\mu^{2}\right)
\end{array}
$$

KKT conditions of lower level problem:

$$
\begin{aligned}
& 0 \leq \lambda+\mu_{1}^{1}+2 \times 50 \sum_{i=1}^{2} q_{i}+610-p_{1} \perp q_{1} \geq 0 \\
& 0 \leq \lambda+\mu_{2}^{1}+2 \times 50 \sum_{i=1}^{2} q_{i}+610-p_{2} \perp q_{2} \geq 0 \\
& 0 \leq \lambda+\mu_{3}^{1}+2 \times 50 \sum_{i=3}^{4} q_{i}+550-p_{3}-W \perp q_{3} \geq 0 \\
& 0 \leq \lambda+\mu_{4}^{1}+2 \times 50 \sum_{i=3}^{4} q_{i}+550-p_{4}-W \perp q_{4} \geq 0 \\
& 0 \leq \lambda+\mu^{2}+2 \times 50 q_{0}+610-1500+2 \times 0.0005 q_{0} \perp q_{0} \geq 0 \\
& 0 \leq Q-\sum_{i=1}^{4} q_{i}-q_{0} \perp \lambda \geq 0 \\
& 0 \leq \bar{q}_{1}-q_{1} \perp \mu_{1}^{1} \geq 0 \\
& 0 \leq \bar{q}_{2}-q_{2} \perp \mu_{2}^{1} \geq 0
\end{aligned}
$$




$$
\begin{aligned}
& 0 \leq \bar{q}_{3}-q_{3} \perp \mu_{3}^{1} \geq 0 \\
& 0 \leq \bar{q}_{4}-q_{4} \perp \mu_{4}^{1} \geq 0 \\
& 0 \leq \bar{q}_{0}-q_{0} \perp \mu^{2} \geq 0
\end{aligned}
$$

\begin{tabular}{|c|c|c|}
\hline $\max _{p_{1}} \pi_{1}=\left(a-b \sum_{i=1}^{4} \beta_{i} q_{i}\right) \times 693 q_{1}-p_{1} q_{1}-890 q_{1}+693 T_{1} q_{1}$ & & (14) \\
\hline $0 \leq p_{1}$ & $\left(\xi_{1}\right)$ & $(15)$ \\
\hline $0 \leq q_{1}$ & $\left(\varepsilon_{11}^{1}\right)$ & $(16.1)$ \\
\hline $0 \leq q_{2}$ & $\left(\varepsilon_{21}^{1}\right)$ & (16.2) \\
\hline $0 \leq q_{3}$ & $\left(\varepsilon_{31}^{1}\right)$ & (16.3) \\
\hline $0 \leq q_{4}$ & $\left(\varepsilon_{41}^{1}\right)$ & (16.4) \\
\hline $0 \leq q_{0}$ & $\left(\varepsilon_{1}^{2}\right)$ & (17) \\
\hline $0 \leq \mu_{1}^{1}$ & $\left(\psi_{11}^{1}\right)$ & (18.1) \\
\hline $0 \leq \mu_{2}^{1}$ & $\left(\psi_{21}^{1}\right)$ & $(18.2)$ \\
\hline $0 \leq \mu_{3}^{1}$ & $\left(\psi_{31}^{1}\right)$ & (18.3) \\
\hline $0 \leq \mu_{4}^{1}$ & $\left(\psi_{41}^{1}\right)$ & (18.4) \\
\hline $0 \leq \mu^{2}$ & $\left(\psi_{1}^{2}\right)$ & (19) \\
\hline $0 \leq \lambda$ & $\left(\psi_{1}^{3}\right)$ & (20) \\
\hline $0 \leq \bar{q}_{1}-q_{1}$ & $\left(\alpha_{11}^{1}\right)$ & (21.1) \\
\hline $0 \leq \bar{q}_{2}-q_{2}$ & $\left(\alpha_{21}^{1}\right)$ & $(21.2)$ \\
\hline $0 \leq \bar{q}_{3}-q_{3}$ & $\left(\alpha_{31}^{1}\right)$ & $(21.3)$ \\
\hline $0 \leq \bar{q}_{4}-q_{4}$ & $\left(\alpha_{41}^{1}\right)$ & (21.4) \\
\hline $0 \leq \bar{q}_{0}-q_{0}$ & $\left(\alpha_{1}^{2}\right)$ & (22) \\
\hline $0 \leq Q-\sum_{i=1}^{4} q_{i}-q_{0}$ & $\left(\alpha_{1}^{3}\right)$ & (23) \\
\hline $0 \leq \lambda+\mu_{1}^{1}+2 \times 50 \sum_{i=1}^{2} q_{i}+610-p_{1}$ & $\left(\delta_{11}^{1}\right)$ & (24.1) \\
\hline $0 \leq \lambda+\mu_{2}^{1}+2 \times 50 \sum_{i=1}^{2} q_{i}+610-p_{2}$ & $\left(\delta_{21}^{1}\right)$ & $(24.2)$ \\
\hline $0 \leq \lambda+\mu_{3}^{1}+2 \times 50 \sum_{i=3}^{4} q_{i}+550-p_{3}-W$ & $\left(\delta_{31}^{1}\right)$ & (25.1) \\
\hline $0 \leq \lambda+\mu_{4}^{1}+2 \times 50 \sum_{i=3}^{4} q_{i}+550-p_{4}-W$ & $\left(\delta_{41}^{1}\right)$ & $(25.2)$ \\
\hline $0 \leq \lambda+\mu^{2}+2 \times 50 q_{0}+610-1500+2 \times 0.0005 q_{0}$ & $\left(\delta_{2}^{2}\right)$ & (26) \\
\hline$q_{1}\left(\lambda+\mu_{1}^{1}+100 \sum_{i=1}^{2} q_{i}+610-p_{1}\right)=0$ & $\left(\theta_{11}^{1}\right)$ & (27.1) \\
\hline$q_{2}\left(\lambda+\mu_{2}^{1}+100 \sum_{i=1}^{2} q_{i}+610-p_{2}\right)=0$ & $\left(\theta_{21}^{1}\right)$ & $(27.2)$ \\
\hline$q_{3}\left(\lambda+\mu_{3}^{1}+100 \sum_{i=3}^{4} q_{i}+550-p_{3}-W\right)=0$ & $\left(\theta_{31}^{1}\right)$ & (28.1) \\
\hline$q_{4}\left(\lambda+\mu_{4}^{1}+100 \sum_{i}^{4} q_{i}+550-p_{4}-W\right)=0$ & $\left(\theta_{41}^{1}\right)$ & (28.2) \\
\hline
\end{tabular}

MPEC for refinery $r=1$ : 


$$
\begin{array}{ll}
x\left(\lambda+\mu^{2}+100 q_{0}+610-1500+0.001 q_{0}\right)=0 & \left(\theta_{1}^{2}\right) \\
\lambda\left(Q-\sum_{i=1}^{4} q_{i}-q_{0}\right)=0 & \left(\varphi_{1}^{3}\right) \\
\mu_{1}^{1}\left(\bar{q}_{1}-q_{1}\right)=0 & \left(\varphi_{11}^{1}\right) \\
\mu_{2}^{1}\left(\bar{q}_{2}-q_{2}\right)=0 & \left(\varphi_{21}^{1}\right) \\
\mu_{3}^{1}\left(\bar{q}_{3}-q_{3}\right)=0 & \left(\varphi_{31}^{1}\right) \\
\mu_{4}^{1}\left(\bar{q}_{4}-q_{4}\right)=0 & \left(\varphi_{41}^{1}\right) \\
\mu^{2}\left(d-q_{0}\right)=0 & \left(\varphi_{1}^{2}\right)
\end{array}
$$

EPEC:

$$
\begin{aligned}
& \frac{\partial L_{1}}{\partial p_{1}}=-q_{1}-\delta_{11}^{1}+\theta_{11}^{1} q_{1}+\xi_{1}=0 \\
& \frac{\partial L_{2}}{\partial p_{2}}=-q_{2}-\delta_{22}^{1}+\theta_{22}^{1} q_{2}+\xi_{2}=0 \\
& \frac{\partial L_{3}}{\partial p_{3}}=-q_{3}-\delta_{33}^{1}+\theta_{33}^{1} q_{3}+\xi_{3}=0 \\
& \frac{\partial L_{4}}{\partial p_{4}}=-q_{4}-\delta_{44}^{1}+\theta_{44}^{1} q_{4}+\xi_{4}=0 \\
& \frac{\partial L_{1}}{\partial q_{1}}=-0.0005 \times 693^{2} q_{1}+693\left(10-0.0005 \sum_{i=1}^{4} \beta_{i} q_{i}\right)-p_{1}-890+\sum_{i=1}^{2} 100 \delta_{i 1}^{1}+\varepsilon_{11}^{1}-\sum_{\substack{i=1 \\
i \neq 1}}^{2} 100 \theta_{i 1}^{1} q_{i} \\
& -\theta_{11}^{1}\left(\lambda+\mu_{1}^{1}+100 \sum_{i=1}^{2} q_{i}+890-p_{1}\right)-100 \theta_{11}^{1} q_{1}-\alpha_{1}^{3}+\varphi_{1}^{3} \lambda-\alpha_{11}^{1}+\varphi_{1}^{2} \mu_{1}^{1}=0 \\
& \frac{\partial L_{2}}{\partial q_{2}}=-0.0005 \times 693^{2} q_{2}+693\left(10-0.0005 \sum_{i=1}^{4} \beta_{i} q_{i}\right)-p_{2}-890+\sum_{i=1}^{2} 100 \delta_{i 2}^{1}+\varepsilon_{22}^{1}-\sum_{\substack{i=1 \\
i \neq 2}}^{2} 100 \theta_{i 2}^{1} q_{i} \\
& -\theta_{22}^{1}\left(\lambda+\mu_{2}^{1}+100 \sum_{i=1}^{2} q_{i}+890-p_{2}\right)-100 \theta_{22}^{1} q_{2}-\alpha_{2}^{3}+\varphi_{2}^{3} \lambda-\alpha_{22}^{1}+\varphi_{2}^{2} \mu_{2}^{1}=0 \\
& \frac{\partial L_{3}}{\partial q_{3}}=-0.0005 \times 330^{2} q_{3}+330\left(10-0.0005 \sum_{i=1}^{4} \beta_{i} q_{i}\right)-p_{3}-1211+330 T+\sum_{i=3}^{4} 100 \delta_{i 3}^{1}+\varepsilon_{33}^{1}-\sum_{\substack{i=3 \\
i \neq 3}}^{4} 100 \theta_{i 3}^{1} q_{i} \\
& -\theta_{33}^{1}\left(\lambda+\mu_{3}^{1}+100 \sum_{i=3}^{4} q_{i}+1211-p_{3}-W\right)-100 \theta_{33}^{1} q_{3}-\alpha_{3}^{3}+\varphi_{3}^{3} \lambda-\alpha_{33}^{1}+\varphi_{3}^{2} \mu_{3}^{1}=0 \\
& \frac{\partial L_{4}}{\partial q_{4}}=-0.0005 \times 330^{2} q_{4}+330\left(10-0.0005 \sum_{i=1}^{4} \beta_{i} q_{i}\right)-p_{4}-1211+330 T+\sum_{i=3}^{4} 100 \delta_{i 4}^{1}+\varepsilon_{44}^{1}-\sum_{\substack{i=3 \\
i \neq 4}}^{4} 100 \theta_{i 4}^{1} q_{i} \\
& -\theta_{44}^{1}\left(\lambda+\mu_{4}^{1}+100 \sum_{i=3}^{4} q_{i}+1211-p_{4}-W\right)-100 \theta_{44}^{1} q_{4}-\alpha_{4}^{3}+\varphi_{4}^{3} \lambda-\alpha_{44}^{1}+\varphi_{4}^{2} \mu_{4}^{1}=0 \\
& \frac{\partial L_{1}}{\partial q_{0}}=0.001 \delta_{1}^{2}+100 \delta_{1}^{2}+\varepsilon_{1}^{2}-\theta_{1}^{2}\left(\lambda+\mu^{2}+100 x+610-1500+0.001 q_{0}\right) \\
& -\theta_{1}^{2} q_{0}(100+0.001)-\alpha_{1}^{3}+\varphi_{1}^{3} \lambda-\alpha_{1}^{2}+\varphi_{1}^{2} \mu^{2}=0 \\
& \frac{\partial L_{2}}{\partial q_{0}}=0.001 \delta_{2}^{2}+100 \delta_{2}^{2}+\varepsilon_{2}^{2}-\theta_{2}^{2}\left(\lambda+\mu^{2}+100 q_{0}+610-1500+0.001 q_{0}\right) \\
& -\theta_{2}^{2} q_{0}(100+0.001)-\alpha_{2}^{3}+\varphi_{2}^{3} \lambda-\alpha_{2}^{2}+\varphi_{2}^{2} \mu^{2}=0 \\
& \frac{\partial L_{3}}{\partial q_{0}}=0.001 \delta_{3}^{2}+100 \delta_{3}^{2}+\varepsilon_{3}^{2}-\theta_{3}^{2}\left(\lambda+\mu^{2}+100 q_{0}+610-1500+0.001 q_{0}\right) \\
& -\theta_{3}^{2} q_{0}(100+0.001)-\alpha_{3}^{3}+\varphi_{3}^{3} \lambda-\alpha_{3}^{2}+\varphi_{3}^{2} \mu^{2}=0 \\
& \frac{\partial L_{4}}{\partial q_{0}}=0.001 \delta_{4}^{2}+100 \delta_{4}^{2}+\varepsilon_{4}^{2}-\theta_{4}^{2}\left(\lambda+\mu^{2}+100 x+610-1500+0.001 q_{0}\right)
\end{aligned}
$$




$$
\begin{aligned}
& -\theta_{4}^{2} q_{0}(100+0.001)-\alpha_{4}^{3}+\varphi_{4}^{3} \lambda-\alpha_{4}^{2}+\varphi_{4}^{2} \mu^{2}=0 \\
& \frac{\partial L_{1}}{\partial \lambda}=\sum_{i=1}^{4} \delta_{i 1}^{1}-\sum_{i=1}^{4} \theta_{i 1}^{1} q_{i}+\delta_{1}^{2}-\theta_{1}^{2} q_{0}+\psi_{1}^{3}-\varphi_{1}^{3} Q=0 \\
& \frac{\partial L_{2}}{\partial \lambda}=\sum_{i=1}^{4} \delta_{i 2}^{1}-\sum_{i=1}^{4} \theta_{i 2}^{1} q_{i}+\delta_{2}^{2}-\theta_{2}^{2} q_{0}+\psi_{2}^{3}-\varphi_{2}^{3} Q=0 \\
& \frac{\partial L_{3}}{\partial \lambda}=\sum_{i=1}^{4} \delta_{i 3}^{1}-\sum_{i=1}^{4} \theta_{i 3}^{1} q_{i}+\delta_{3}^{2}-\theta_{3}^{2} q_{0}+\psi_{3}^{3}-\varphi_{3}^{3} Q=0 \\
& \frac{\partial L_{4}}{\partial \lambda}=\sum_{i=1}^{4} \delta_{i 4}^{1}-\sum_{i=1}^{4} \theta_{i 4}^{1} q_{i}+\delta_{4}^{2}-\theta_{4}^{2} q_{0}+\psi_{4}^{3}-\varphi_{4}^{3} Q=0 \\
& \frac{\partial L_{1}}{\partial \mu_{1}^{1}}=\delta_{11}^{1}-\theta_{11}^{1} q_{1}+\psi_{11}^{1}-\varphi_{11}^{1}\left(\bar{q}_{1}-q_{1}\right)=0 \\
& \frac{\partial L_{1}}{\partial \mu_{2}^{1}}=\delta_{21}^{1}-\theta_{21}^{1} q_{2}+\psi_{21}^{1}-\varphi_{21}^{1}\left(\bar{q}_{2}-q_{2}\right)=0 \\
& \frac{\partial L_{1}}{\partial \mu_{3}^{1}}=\delta_{31}^{1}-\theta_{31}^{1} q_{3}+\psi_{31}^{1}-\varphi_{31}^{1}\left(\bar{q}_{3}-q_{3}\right)=0 \\
& \frac{\partial L_{1}}{\partial \mu_{4}^{1}}=\delta_{41}^{1}-\theta_{41}^{1} q_{4}+\psi_{41}^{1}-\varphi_{41}^{1}\left(\bar{q}_{4}-q_{4}\right)=0 \\
& \frac{\partial L_{2}}{\partial \mu_{1}^{1}}=\delta_{12}^{1}-\theta_{12}^{1} q_{1}+\psi_{12}^{1}-\varphi_{12}^{1}\left(\bar{q}_{1}-q_{1}\right)=0 \\
& \frac{\partial L_{2}}{\partial \mu_{2}^{1}}=\delta_{22}^{1}-\theta_{22}^{1} q_{2}+\psi_{22}^{1}-\varphi_{22}^{1}\left(\bar{q}_{2}-q_{2}\right)=0 \\
& \frac{\partial L_{2}}{\partial \mu_{3}^{1}}=\delta_{32}^{1}-\theta_{32}^{1} q_{3}+\psi_{32}^{1}-\varphi_{32}^{1}\left(\bar{q}_{3}-q_{3}\right)=0 \\
& \frac{\partial L_{2}}{\partial \mu_{4}^{1}}=\delta_{42}^{1}-\theta_{42}^{1} q_{4}+\psi_{42}^{1}-\varphi_{42}^{1}\left(\bar{q}_{4}-q_{4}\right)=0 \\
& \frac{\partial L_{3}}{\partial \mu_{1}^{1}}=\delta_{13}^{1}-\theta_{13}^{1} q_{1}+\psi_{13}^{1}-\varphi_{13}^{1}\left(\bar{q}_{1}-q_{1}\right)=0 \\
& \frac{\partial L_{3}}{\partial \mu_{2}^{1}}=\delta_{23}^{1}-\theta_{23}^{1} q_{2}+\psi_{23}^{1}-\varphi_{23}^{1}\left(\bar{q}_{2}-q_{2}\right)=0 \\
& \frac{\partial L_{3}}{\partial \mu_{3}^{1}}=\delta_{33}^{1}-\theta_{33}^{1} q_{3}+\psi_{33}^{1}-\varphi_{33}^{1}\left(\bar{q}_{3}-q_{3}\right)=0 \\
& \frac{\partial L_{3}}{\partial \mu_{4}^{1}}=\delta_{43}^{1}-\theta_{43}^{1} q_{4}+\psi_{43}^{1}-\varphi_{43}^{1}\left(\bar{q}_{4}-q_{4}\right)=0 \\
& \frac{\partial L_{4}}{\partial \mu_{1}^{1}}=\delta_{14}^{1}-\theta_{14}^{1} q_{1}+\psi_{14}^{1}-\varphi_{14}^{1}\left(\bar{q}_{1}-q_{1}\right)=0 \\
& \frac{\partial L_{4}}{\partial \mu_{2}^{1}}=\delta_{24}^{1}-\theta_{24}^{1} q_{2}+\psi_{24}^{1}-\varphi_{24}^{1}\left(\bar{q}_{2}-q_{2}\right)=0 \\
& \frac{\partial L_{4}}{\partial \mu_{3}^{1}}=\delta_{34}^{1}-\theta_{34}^{1} q_{3}+\psi_{34}^{1}-\varphi_{34}^{1}\left(\bar{q}_{3}-q_{3}\right)=0 \\
& \frac{\partial L_{4}}{\partial \mu_{4}^{1}}=\delta_{44}^{1}-\theta_{44}^{1} q_{4}+\psi_{44}^{1}-\varphi_{44}^{1}\left(\bar{q}_{4}-q_{4}\right)=0 \\
& \frac{\partial L_{1}}{\partial \mu^{2}}=\delta_{1}^{2}-\theta_{1}^{2} q_{0}+\psi_{1}^{2}-\varphi_{1}^{2}\left(\bar{q}_{0}-q_{0}\right)=0 \\
& \frac{\partial L_{2}}{\partial \mu^{2}}=\delta_{2}^{2}-\theta_{2}^{2} q_{0}+\psi_{2}^{2}-\varphi_{2}^{2}\left(\bar{q}_{0}-q_{0}\right)=0 \\
& \frac{\partial L_{3}}{\partial \mu^{2}}=\delta_{3}^{2}-\theta_{3}^{2} q_{0}+\psi_{3}^{2}-\varphi_{3}^{2}\left(\bar{q}_{0}-q_{0}\right)=0 \\
& \frac{\partial L_{4}}{\partial \mu^{2}}=\delta_{4}^{2}-\theta_{4}^{2} q_{0}+\psi_{4}^{2}-\varphi_{4}^{2}\left(\bar{q}_{0}-q_{0}\right)=0
\end{aligned}
$$




$$
\begin{aligned}
& 0 \leq \delta_{11}^{1} \perp\left(\lambda+\mu_{1}^{1}+100 \sum_{i=1}^{2} q_{i}+610-p_{1}\right) \geq 0 \\
& 0 \leq \delta_{21}^{1} \perp\left(\lambda+\mu_{2}^{1}+100 \sum_{i=1}^{2} q_{i}+610-p_{2}\right) \geq 0 \\
& 0 \leq \delta_{12}^{1} \perp\left(\lambda+\mu_{1}^{1}+100 \sum_{i=1}^{2} q_{i}+610-p_{1}\right) \geq 0 \\
& 0 \leq \delta_{22}^{1} \perp\left(\lambda+\mu_{2}^{1}+100 \sum_{i=1}^{2} q_{i}+610-p_{2}\right) \geq 0 \\
& 0 \leq \delta_{13}^{1} \perp\left(\lambda+\mu_{1}^{1}+100 \sum_{i=1}^{2} q_{i}+610-p_{1}\right) \geq 0 \\
& 0 \leq \delta_{23}^{1} \perp\left(\lambda+\mu_{2}^{1}+100 \sum_{i=1}^{2} q_{i}+610-p_{2}\right) \geq 0 \\
& 0 \leq \delta_{14}^{1} \perp\left(\lambda+\mu_{1}^{1}+100 \sum_{i=1}^{2} q_{i}+610-p_{1}\right) \geq 0 \\
& 0 \leq \delta_{24}^{1} \perp\left(\lambda+\mu_{2}^{1}+100 \sum_{i=1}^{2} q_{i}+610-p_{2}\right) \geq 0 \\
& 0 \leq \delta_{31}^{1} \perp\left(\lambda+\mu_{3}^{1}+100 \sum_{i=3}^{4} q_{i}+550-p_{3}-W\right) \geq 0 \\
& 0 \leq \delta_{41}^{1} \perp\left(\lambda+\mu_{4}^{1}+100 \sum_{i=3}^{4} q_{i}+550-p_{4}-W\right) \geq 0 \\
& 0 \leq \delta_{32}^{1} \perp\left(\lambda+\mu_{3}^{1}+100 \sum_{i=3}^{4} q_{i}+550-p_{3}-W\right) \geq 0 \\
& 0 \leq \delta_{42}^{1} \perp\left(\lambda+\mu_{4}^{1}+100 \sum_{i=3}^{4} q_{i}+550-p_{4}-W\right) \geq 0 \\
& 0 \leq \delta_{33}^{1} \perp\left(\lambda+\mu_{3}^{1}+100 \sum_{i=3}^{4} q_{i}+550-p_{3}-W\right) \geq 0 \\
& 0 \leq \delta_{43}^{1} \perp\left(\lambda+\mu_{4}^{1}+100 \sum_{i=3}^{4} q_{i}+550-p_{4}-W\right) \geq 0 \\
& 0 \leq \delta_{34}^{1} \perp\left(\lambda+\mu_{3}^{1}+100 \sum_{i=3}^{4} q_{i}+550-p_{3}-W\right) \geq 0 \\
& 0 \leq \delta_{44}^{1} \perp\left(\lambda+\mu_{4}^{1}+100 \sum_{i=3}^{4} q_{i}+550-p_{4}-W\right) \geq 0 \\
& 0 \leq \delta_{1}^{2} \perp\left(\lambda+\mu^{2}+100 \bar{q}_{0}+610-1500+0.001 \bar{q}_{0}\right) \geq 0 \mathrm{~s} \\
& 0 \leq \delta_{2}^{2} \perp\left(\lambda+\mu^{2}+100 \bar{q}_{0}+610-1500+0.001 \bar{q}_{0}\right) \geq 0 \\
& 0 \leq \delta_{3}^{2} \perp\left(\lambda+\mu^{2}+100 \bar{q}_{0}+610-1500+0.001 \bar{q}_{0}\right) \geq 0 \\
& 0 \leq \delta_{4}^{2} \perp\left(\lambda+\mu^{2}+100 \bar{q}_{0}+610-1500+0.001 \bar{q}_{0}\right) \geq 0 \\
& 0 \leq \xi_{1} \perp p_{1} \geq 0 \\
& 0 \leq \xi_{2} \perp p_{2} \geq 0 \\
& 0 \leq \xi_{3} \perp p_{3} \geq 0 \\
& 0 \leq \xi_{4} \perp p_{4} \geq 0 \\
& 0 \leq \varepsilon_{11}^{1} \perp q_{1} \geq 0 \\
& 0 \leq \varepsilon_{21}^{1} \perp q_{2} \geq 0 \\
& 0 \leq \varepsilon_{31}^{1} \perp q_{3} \geq 0 \\
& 0 \leq \varepsilon_{41}^{1} \perp q_{4} \geq 0 \\
& 0 \leq \varepsilon_{12}^{1} \perp q_{1} \geq 0 \\
& 0 \leq \varepsilon_{22}^{1} \perp q_{2} \geq 0
\end{aligned}
$$




\begin{tabular}{|c|c|}
\hline $0 \leq \varepsilon_{32}^{1} \perp q_{3} \geq 0$ & $(44.7)$ \\
\hline $0 \leq \varepsilon_{42}^{1} \perp q_{4} \geq 0$ & $(44.8)$ \\
\hline $0 \leq \varepsilon_{13}^{1} \perp q_{1} \geq 0$ & $(44.9)$ \\
\hline $0 \leq \varepsilon_{23}^{1} \perp q_{2} \geq 0$ & $(44.10)$ \\
\hline $0 \leq \varepsilon_{33}^{1} \perp q_{3} \geq 0$ & $(44.11)$ \\
\hline $0 \leq \varepsilon_{43}^{1} \perp q_{4} \geq 0$ & $(44.12)$ \\
\hline $0 \leq \varepsilon_{14}^{1} \perp q_{1} \geq 0$ & $(44.13)$ \\
\hline $0 \leq \varepsilon_{24}^{1} \perp q_{2} \geq 0$ & $(44.14)$ \\
\hline $0 \leq \varepsilon_{34}^{1} \perp q_{3} \geq 0$ & $(44.15)$ \\
\hline $0 \leq \varepsilon_{44}^{1} \perp q_{4} \geq 0$ & $(44.16)$ \\
\hline $0 \leq \varepsilon_{1}^{2} \perp \bar{q}_{0} \geq 0$ & $(45.1)$ \\
\hline $0 \leq \varepsilon_{2}^{2} \perp \bar{q}_{0} \geq 0$ & $(45.2)$ \\
\hline $0 \leq \varepsilon_{4}^{2} \perp \bar{q}_{0} \geq 0$ & $(45.4)$ \\
\hline $0 \leq \psi_{11}^{1} \perp \mu_{1}^{1} \geq 0$ & $(46.1)$ \\
\hline $0 \leq \psi_{21}^{1} \perp \mu_{2}^{1} \geq 0$ & $(46.2)$ \\
\hline $0 \leq \psi_{31}^{1} \perp \mu_{3}^{1} \geq 0$ & $(46.3)$ \\
\hline $0 \leq \psi_{41}^{1} \perp \mu_{4}^{1} \geq 0$ & $(46.4)$ \\
\hline $0 \leq \psi_{12}^{1} \perp \mu_{1}^{1} \geq 0$ & $(46.5)$ \\
\hline $0 \leq \psi_{22}^{1} \perp \mu_{2}^{1} \geq 0$ & $(46.6)$ \\
\hline $0 \leq \psi_{32}^{1} \perp \mu_{3}^{1} \geq 0$ & $(46.7)$ \\
\hline $0 \leq \psi_{42}^{1} \perp \mu_{4}^{1} \geq 0$ & $(46.8)$ \\
\hline $0 \leq \psi_{13}^{1} \perp \mu_{1}^{1} \geq 0$ & $(46.9)$ \\
\hline $0 \leq \psi_{23}^{1} \perp \mu_{2}^{1} \geq 0$ & $(46.10)$ \\
\hline $0 \leq \psi_{33}^{1} \perp \mu_{3}^{1} \geq 0$ & $(46.11)$ \\
\hline $0 \leq \psi_{43}^{1} \perp \mu_{4}^{1} \geq 0$ & $(46.12)$ \\
\hline $0 \leq \psi_{14}^{1} \perp \mu_{1}^{1} \geq 0$ & $(46.13)$ \\
\hline $0 \leq \psi_{24}^{1} \perp \mu_{2}^{1} \geq 0$ & $(46.14)$ \\
\hline $0 \leq \psi_{34}^{1} \perp \mu_{3}^{1} \geq 0$ & $(46.15)$ \\
\hline $0 \leq \psi_{44}^{1} \perp \mu_{4}^{1} \geq 0$ & $(46.16)$ \\
\hline $0 \leq \psi_{1}^{2} \perp \mu^{2} \geq 0$ & $(47.1)$ \\
\hline $0 \leq \psi_{2}^{2} \perp \mu^{2} \geq 0$ & $(47.2)$ \\
\hline $0 \leq \psi_{3}^{2} \perp \mu^{2} \geq 0$ & $(47.3)$ \\
\hline $0 \leq \psi_{4}^{2} \perp \mu^{2} \geq 0$ & $(47.4)$ \\
\hline $0 \leq \alpha_{1}^{3} \perp\left(Q-\sum_{i=1}^{4} q_{i}-q_{0}\right) \geq 0$ & $(48.1)$ \\
\hline $0 \leq \alpha_{2}^{3} \perp\left(Q-\sum_{i=1}^{4} q_{i}-q_{0}\right) \geq 0$ & $(48.2)$ \\
\hline $0 \leq \alpha_{3}^{3} \perp\left(Q-\sum_{i=1}^{4} q_{i}-q_{0}\right) \geq 0$ & $(48.3)$ \\
\hline $0 \leq \alpha_{4}^{3} \perp\left(Q-\sum^{4} q_{i}-q_{0}\right) \geq 0$ & $(48.4)$ \\
\hline
\end{tabular}




$$
\begin{aligned}
& 0 \leq \alpha_{1}^{2} \perp\left(\bar{q}_{0}-q_{0}\right) \geq 0 \\
& 0 \leq \alpha_{2}^{2} \perp\left(\bar{q}_{0}-q_{0}\right) \geq 0 \\
& 0 \leq \alpha_{3}^{2} \perp\left(\bar{q}_{0}-q_{0}\right) \geq 0 \\
& 0 \leq \alpha_{4}^{2} \perp\left(\bar{q}_{0}-q_{0}\right) \geq 0 \\
& 0 \leq \alpha_{11}^{1} \perp\left(\bar{q}_{1}-q_{1}\right) \geq 0 \\
& 0 \leq \alpha_{21}^{1} \perp\left(\bar{q}_{2}-q_{2}\right) \geq 0 \\
& 0 \leq \alpha_{31}^{1} \perp\left(\bar{q}_{3}-q_{3}\right) \geq 0 \\
& 0 \leq \alpha_{41}^{1} \perp\left(\bar{q}_{4}-q_{4}\right) \geq 0 \\
& 0 \leq \alpha_{12}^{1} \perp\left(\bar{q}_{1}-q_{1}\right) \geq 0 \\
& 0 \leq \alpha_{22}^{1} \perp\left(\bar{q}_{2}-q_{2}\right) \geq 0 \\
& 0 \leq \alpha_{32}^{1} \perp\left(\bar{q}_{3}-q_{3}\right) \geq 0 \\
& 0 \leq \alpha_{42}^{1} \perp\left(\bar{q}_{4}-q_{4}\right) \geq 0 \\
& 0 \leq \alpha_{13}^{1} \perp\left(\bar{q}_{1}-q_{1}\right) \geq 0 \\
& 0 \leq \alpha_{23}^{1} \perp\left(\bar{q}_{2}-q_{2}\right) \geq 0 \\
& 0 \leq \alpha_{33}^{1} \perp\left(\bar{q}_{3}-q_{3}\right) \geq 0 \\
& 0 \leq \alpha_{43}^{1} \perp\left(\bar{q}_{4}-q_{4}\right) \geq 0 \\
& 0 \leq \alpha_{14}^{1} \perp\left(\bar{q}_{1}-q_{1}\right) \geq 0 \\
& 0 \leq \alpha_{24}^{1} \perp\left(\bar{q}_{2}-q_{2}\right) \geq 0 \\
& 0 \leq \alpha_{34}^{1} \perp\left(\bar{q}_{3}-q_{3}\right) \geq 0 \\
& 0 \leq \alpha_{44}^{1} \perp\left(\bar{q}_{4}-q_{4}\right) \geq 0 \\
& 0 \leq \psi_{1}^{3} \perp \lambda \geq 0 \\
& 0 \leq \psi_{2}^{3} \perp \lambda \geq 0 \\
& 0 \leq \psi_{3}^{3} \perp \lambda \geq 0 \\
& 0 \leq \psi_{4}^{3} \perp \lambda \geq 0 \\
&
\end{aligned}
$$




\section{Appendix B.2}

The EPEC in section 4.2.5 has the following nonlinearities:

1. The complementarity conditions (40)-(51)

2. The term $\theta_{r r}^{1} q_{r}$ in (33)-(35) and (37)-(38)

3. The term $\theta_{r r}^{1} \lambda$ in (34)-(35)

4. The term $\theta_{r r}^{1} \mu_{r}^{1}$ in (34)-(35)

5. The term $\theta_{r r}^{1} p_{r}$ in (34)-(35)

6. The term $\theta_{r}^{2} x$ in (36)-(37) and (39)

7. The term $\theta_{r}^{2} \lambda$ in (36)

8. The term $\theta_{r}^{2} \mu^{2}$ in (36)

9. The term $\varphi_{r}^{3} \lambda$ in (34)-(36)

10. The term $\varphi_{r}^{2} x$ in (39)

11. The term $\varphi_{r}^{2} \mu_{r}^{1}$ in (34)-(36)

12. The term $\varphi_{i r}^{1} q_{i}$ in (38)

Linearization of constrains (33):

$$
\begin{aligned}
& \frac{\partial L_{r}}{\partial p_{r}}=-q_{r}-\delta_{r r}^{1}+\Delta_{\theta 1} \sum_{f} 2^{f} z_{f r r}^{\theta 1, q}+\xi_{r}=0 \quad r \in I \\
& 0 \leq z_{f r r}^{\theta 1, q} \leq M b_{f r r}^{\theta 1, q} \\
& 0 \leq q_{r}-z_{f r r}^{\theta 1, q} \leq M\left(1-b_{f r r}^{\theta 1, q}\right)
\end{aligned}
$$

Linearization of constraints (34)-(35):

$$
\begin{aligned}
& \frac{\partial L_{r}}{\partial q_{r}}=-b \beta_{r}^{2} q_{r}+\beta_{r}\left(a-b \sum_{i \in I} \beta_{i} q_{i}\right)-p_{r}-c_{r}^{R}+T_{r} \beta_{r}-c_{r}^{1}-c_{r}^{2}+\sum_{i \in J} 2 c^{e 1} \delta_{i r}^{1}+\varepsilon_{r r}^{1}-2 c^{e 1} \Delta_{\theta 1} \sum_{\substack{i \in J \\
i \neq r}} \sum_{f}^{f} z_{f i r}^{\theta l, q} \\
& -\Delta_{\theta 1} \sum_{f} 2^{f}\left(z_{f i r}^{\theta 1, \lambda}+z_{f r r}^{\theta 1, \mu 1}+2 c^{e 1} \sum_{i \in J} z_{f i r}^{\theta 1, q}+c_{r}^{f}-z_{f r r}^{\theta 1, p}\right)-2 c^{e 1} \Delta_{\theta 1} \sum_{f} 2^{f} z_{f i r}^{\theta 1, q}-\alpha_{r}^{3}+\Delta_{\varphi 3} \sum_{f} 2^{f} z_{f r}^{\varphi, 3}-\alpha_{r r}^{1}+\Delta_{\varphi 2} \sum_{f} 2^{f} z_{f r}^{\varphi 2, \mu 1}=0 \quad r \in J \\
& 0 \leq z_{f i r}^{\theta 1, q} \leq M b_{f i r}^{\theta 1, q} \\
& 0 \leq q_{r}-z_{f i r}^{\theta 1, q} \leq M\left(1-b_{f i r}^{\theta 1, q}\right) \\
& 0 \leq z_{\text {fri }}^{\theta 1, \lambda} \leq M b_{f r r}^{\theta 1, \lambda} \\
& 0 \leq \lambda-z_{\text {frr }}^{\theta 1, \lambda} \leq M\left(1-b_{f r r}^{\theta 1, \lambda}\right) \\
& 0 \leq z_{f r r}^{\theta 1, \mu 1} \leq M b_{f r r}^{\theta 1, \mu 1} \\
& 0 \leq \mu_{r}^{1}-z_{f r r}^{\theta 1, \mu 1} \leq M\left(1-b_{f r r}^{\theta 1, \mu 1}\right) \\
& 0 \leq z_{\text {frr }}^{\theta 1, p} \leq M b_{\text {frr }}^{\theta 1, p} \\
& 0 \leq p_{r}-z_{f i r}^{\theta 1, p} \leq M\left(1-b_{f i r}^{\theta 1, p}\right) \\
& 0 \leq z_{f r}^{\varphi 3, \lambda} \leq M b_{f r}^{\varphi 3, \lambda} \\
& 0 \leq \lambda-z_{f r}^{\varphi 3, \lambda} \leq M\left(1-b_{f r}^{\varphi 3, \lambda}\right) \\
& 0 \leq z_{f r}^{\varphi 2, \mu 1} \leq M b_{f r}^{\varphi 2, \mu 1} \\
& 0 \leq \mu_{r}^{1}-z_{f r}^{\varphi 2, \mu 1} \leq M\left(1-b_{f r}^{\varphi 2, \mu 1}\right)
\end{aligned}
$$

Linearization of constraints (36):

$\frac{\partial L_{r}}{\partial x}=2 \delta_{r}^{2} b_{0}+2 c^{e 2} \delta_{r}^{2}+\varepsilon_{r}^{2}-\Delta_{\theta 2} \sum_{f} 2^{f}\left(z_{f r}^{\theta 2, \lambda}+z_{f r}^{\theta 2, \mu 2}+2 c^{e 2} z_{f r}^{\theta 2, q_{0}}+c_{1}^{f}-a_{0}+2 b_{0} z_{f r}^{\theta 2, q_{0}}\right)$ 


$$
\begin{aligned}
& -\Delta_{\theta 2} \sum_{f} 2^{f} z_{f r}^{\theta 2, q_{0}}\left(2 c^{e 2}+2 b_{0}\right)-\alpha_{r}^{3}+\Delta_{\varphi 3} \sum_{f} 2^{f} z_{f r}^{\varphi 3, \lambda}-\alpha_{r}^{2}+\Delta_{\varphi 2} \sum_{f} 2^{f} z_{f r}^{\varphi 2, \mu}=0 \\
& 0 \leq z_{f r}^{\theta 2, \lambda} \leq M b_{f r}^{\theta 2, \lambda} \\
& 0 \leq \lambda-z_{f r}^{\theta 2, \lambda} \leq M\left(1-b_{f r}^{\theta 2, \lambda}\right) \\
& 0 \leq z_{f r}^{\theta 2, \mu 2} \leq M b_{f r}^{\theta 2, \mu 2} \\
& 0 \leq \mu^{2}-z_{f r}^{\theta 2, \mu 2} \leq M\left(1-b_{f r}^{\theta 2, \mu 2}\right) \\
& 0 \leq z_{f r}^{\theta 2, q_{0}} \leq M b_{f r}^{\theta 2, q_{0}} \\
& 0 \leq q_{0}-z_{f r}^{\theta 2, q_{0}} \leq M\left(1-b_{f r}^{\theta 2, q_{0}}\right) \\
& 0 \leq z_{f r}^{\varphi 3, \lambda} \leq M b_{f r}^{\varphi 3, \lambda} \\
& 0 \leq x-z_{f r}^{\varphi 3, \lambda} \leq M\left(1-b_{f r}^{\varphi 3, \lambda}\right) \\
& 0 \leq z_{f r}^{\varphi 2, \mu 2} \leq M b_{f r}^{\varphi 2, \mu 2} \\
& 0 \leq x-z_{f r}^{\varphi 2, \mu 2} \leq M\left(1-b_{f r}^{\varphi 2, \mu 2}\right)
\end{aligned}
$$

Linearization of constraints (37):

$$
\begin{aligned}
& \frac{\partial L_{r}}{\partial \lambda}=\sum_{i \in I} \delta_{i r}^{1}-\Delta_{\theta 1} \sum_{\substack{i \in l \\
i \neq r}} \sum_{f}^{f f} z_{f i r}^{\theta 1, q}+\delta_{r}^{2}-\Delta_{\theta 2} \sum_{f} 2^{f} z_{f r r}^{\theta 2, q_{0}}+\psi_{r}^{3}-\varphi_{r}^{3} Q=0 \quad r \in I \\
& 0 \leq z_{f f r}^{\theta 1, q} \leq M b_{f i r}^{\theta \theta, q} \\
& 0 \leq q_{i}-z_{f i r}^{\theta 1, q} \leq M\left(1-b_{f i r}^{\theta 1, q}\right) \\
& 0 \leq z_{f r}^{\theta 2, q_{0}} \leq M b_{f r}^{\theta 2, q_{0}} \\
& 0 \leq q_{i}-z_{f r}^{\theta 2, q_{0}} \leq M\left(1-b_{f r}^{\theta 2, x}\right)
\end{aligned}
$$

Linearization of constraints (38):

$$
\begin{aligned}
& \frac{\partial L_{r}}{\partial \mu_{i}^{1}}=\delta_{i r}^{1}-\Delta_{\theta 1} \sum_{f} 2^{f} z_{f i r}^{\theta 1, q}+\psi_{i r}^{1}-\Delta_{\varphi 1} \sum_{f} 2^{f}\left(\bar{q}_{i}-z_{f i r}^{\varphi 1, q}\right)=0 \quad \forall r, i \in I \\
& 0 \leq z_{f i r}^{\varphi 1, q} \leq M b_{f i r}^{\varphi 1, q} \\
& 0 \leq q_{i}-z_{f i r}^{\varphi 1, q} \leq M\left(1-b_{f i r}^{\varphi 1, q}\right)
\end{aligned}
$$

Linearization of constraints (39):

$\frac{\partial L_{r}}{\partial \mu^{2}}=\delta_{r}^{2}-\Delta_{\theta 2} \sum_{f} 2^{f} z_{f r}^{\theta 2, q_{0}}+\psi_{r}^{2}-\Delta_{\varphi 2} \sum_{f} 2^{f} z_{f r}^{\varphi 2, q_{0}} d+\Delta_{\varphi 2} \sum_{f} 2^{f} z_{f r}^{\varphi 2, q_{0}}=0 \quad \forall r \in I$

Linearization of constraints (40):

$$
\begin{array}{ll}
\lambda+\mu_{i}^{1}+2 c^{e 1} \sum_{j \in J} q_{j}+c_{i}^{f}-p_{i} \geq 0 & i \in J \\
\delta_{i r}^{1} \geq 0 & i \in J \\
\lambda+\mu_{i}^{1}+2 c^{e 1} \sum_{j \in J} q_{j}+c_{i}^{f}-p_{i} \leq\left(1-\omega_{i r}\right) M^{1} & i \in J \\
\delta_{i r}^{1} \leq \omega_{i r} M^{1} & i \in J
\end{array}
$$

Linearization of constraints (41):

$$
\begin{array}{ll}
\lambda+\mu_{i}^{1}+2 c^{e 2} \sum_{k \in K} q_{k}+c_{i}^{f}-p_{i}-W \geq 0 & i \in K \\
\delta_{i r}^{1} \geq 0 & i \in K \\
\lambda+\mu_{i}^{1}+2 c^{e 2} \sum_{k \in K} q_{k}+c_{i}^{f}-p_{i}-W \leq\left(1-\omega_{i r}\right) M^{1} & i \in K \\
\delta_{i r}^{1} \leq \omega_{i r} M^{1} & i \in K
\end{array}
$$

Linearization of constraints (42): 
$\lambda+\mu^{2}+2 c^{e 2} q_{0}+c_{1}^{f}-a_{0}+2 b_{0} q_{0} \geq 0$

$\delta_{r}^{2} \geq 0$

$\lambda+\mu^{2}+2 c^{e 2} q_{0}+c_{1}^{f}-a_{0}+2 b_{0} q_{0} \leq\left(1-\omega_{r}^{2}\right) M^{2}$

$\delta_{r}^{2} \leq \omega_{r}^{2} M^{2}$

Linearization of constraints (43):

$p_{r} \geq 0$

$\xi_{r} \geq 0$

$p_{r} \leq\left(1-\omega_{r}^{3}\right) M^{3}$

$\xi_{r} \leq \omega_{r}^{3} M^{3}$

Linearization of constraints (44):

$q_{i} \geq 0$

$\varepsilon_{i r}^{1} \geq 0$

$q_{i} \leq\left(1-\omega_{i r}^{4}\right) M^{4}$

$\varepsilon_{i r}^{1} \leq \omega_{i r}^{4} M^{4}$

Linearization of constraints (45):

$q_{0} \geq 0$

$\varepsilon_{r}^{2} \geq 0$

$x \leq\left(1-\omega_{r}^{5}\right) M^{5}$

$\varepsilon_{r}^{2} \leq \omega_{r}^{5} M^{5}$

Linearization of constraints (46):

$\mu_{i}^{1} \geq 0$

$\psi_{i r}^{1} \geq 0$

$\mu_{i}^{1} \leq\left(1-\omega_{i r}^{6}\right) M^{6}$

$\psi_{i r}^{1} \leq \omega_{i r}^{6} M^{6}$

Linearization of constraints (47):

$\mu^{2} \geq 0$

$\psi_{r}^{2} \geq 0$

$\mu^{2} \leq\left(1-\omega_{r}^{7}\right) M^{7}$

$\psi_{r}^{2} \leq \omega_{r}^{7} M^{7}$

Linearization of constraints (48):

$\left(Q-\sum_{i \in I} q_{i}-q_{0}\right) \leq\left(1-\omega_{r}^{14}\right) M^{14}$

$\lambda \leq \omega_{r}^{14} M^{14}$

Linearization of constraints (49):

$\left(Q-\sum_{i \in I} q_{i}-q_{0}\right) \geq 0$

$\alpha_{r}^{3} \geq 0$ 
$\left(Q-\sum_{i \in I} q_{i}-q_{0}\right) \leq\left(1-\omega_{r}^{8}\right) M^{8}$

$\alpha_{r}^{3} \leq \omega_{r}^{8} M^{8}$

Linearization of constraints (50):

$\left(\bar{q}_{0}-q_{0}\right) \geq 0$

$\alpha_{r}^{2} \geq \mathbf{O}$

$\left(\bar{q}_{0}-q_{0}\right) \leq\left(1-\omega_{r}^{9}\right) M^{9}$

$\alpha_{r}^{2} \leq \omega_{r}^{9} M^{9}$

Linearization of constraints (51):

$\left(\bar{q}_{i}-q_{i}\right) \geq 0$

$\alpha_{i r}^{1} \geq 0$

$\left(\bar{q}_{i}-q_{i}\right) \leq\left(1-\omega_{i r}^{10}\right) M^{10}$

$\alpha_{i r}^{1} \leq \omega_{i r}^{10} M^{10}$

Linearization of constraints (52.1):

$\lambda \geq 0$

$\psi_{r}^{3} \geq 0$

$\lambda \leq\left(1-\omega_{r}^{11}\right) M^{11}$

$\psi_{r}^{3} \leq \omega_{r}^{11} M^{11}$

Linearization of constraints (52.2):

$\left(\lambda+\mu_{j}^{1}+2 c^{e 1} \sum_{i \in J} q_{i}+c_{j}^{f}-p_{j}\right) \leq\left(1-\omega_{j r}^{12}\right) M^{12}$

$q_{j} \leq \omega_{j r}^{12} M^{12}$

Linearization of constraints (52.3):

$\left(\lambda+\mu_{k}^{1}+2 c^{e 2} \sum_{i \in k} q_{i}+c_{k}^{f}-p_{k}-W\right) \leq\left(1-\omega_{k r}^{12}\right) M^{12}$

$q_{k} \leq \omega_{k r}^{12} M^{12}$

Linearization of constraints (52.4):

$\left(\lambda+\mu^{2}+2 c^{e 1} q_{0}+c_{1}^{f}-a_{0}+2 b_{0} q_{0}\right) \leq\left(1-\omega_{r}^{13}\right) M^{13}$

$q_{0} \leq \omega_{r}^{13} M^{13}$

Linearization of constraints (52.5):

$\bar{q}_{i}-q_{i} \leq\left(1-\omega_{i r}^{15}\right) M^{15}$

$\mu_{i}^{1} \leq \omega_{i r}^{15} M^{15}$

Linearization of constraints (52.6):

$\bar{q}_{0}-q_{0} \leq\left(1-\omega_{r}^{16}\right) M^{16}$

$\mu^{2} \leq \omega_{r}^{16} M^{16}$ 


\section{Curriculum Vitae}

\section{EDUCATION}

\section{University of Wisconsin-Milwaukee}

Ph.D. in Operations Research

Minor: Business Statistics

Sharif University of Technology

M.Sc. in Industrial Engineering

Amirkabir University of Technology

B.Sc. in Industrial and Systems Engineering
Milwaukee, WI

Fall 2014 - Fall 2020 | GPA: 3.85

Tehran

Fall 2010 - Fall 2012

Tehran

Fall 2006 - Spring 2010

\section{RESEARCH INTEREST}

- Applied Operations Research/Game Theory

- Operations Management

- Statistics

\section{PUBLICATIONS AND PRESENTATIONS}

- Pichka, K., Bajgiran, A., Jang, J., (2018), The two-echelon open location routing problem: Mathematical model and hybrid heuristic, Journal of Computers and Industrial Engineering.

- Bajgiran, A, (2016), Game-theoretic modeling of a biofuel supply chain with existence of incentives, Presentation at INFORMS Annual Meeting.

- Bajgiran, A., Jang, J., Fang, X., Peoples, J., (2018), Game-theoretic modeling of a biofuel supply chain with existence of incentives, International Journal of Production Economics, Under the first review step.

- Bajgiran, A., Mardi, M., Soofi, E., (2018), Consistent Maximum Entropy Newsvendor Demand Distribution, International Journal of Operations Research, Under the first review step.

- Jang, J., Bajgiran, A., (2018), Strategic Inventory management with Cournot competition, Production and Operations Management, Under the first review step.

- Bajgiran, A., Mardi, M., Soofi, E., Maximum entropy demand models with newsvendor information, to be presented by Prof. Soofi at the invited session "Statistics in Operations" of International Symposium on Business and Industrial Statistics (ISBIS Meeting), July 2-4, 2018, Greece

\section{TEACHING EXPERIENCE}

University of Wisconsin Milwaukee

Milwaukee, WI

Instructor / Graduate Teaching Assistant, Statistics for Engineering Students, Aug 2014 - Present

\section{HONORS AND AWARDS}

- NSF Grant: Full financial support to attend the 34th Quality and Productivity Research Conference to present a poster with the title of "Maximum entropy newsvendor models", June 2017

- Chancellor's Graduate Student Award: University of Wisconsin-Milwaukee, summer 2016- 2017 
- UWM Travel Fellowship Award: Full financial support to attend the INFORMS Annual Meeting on Business Analytics \& Operations Research, fall 2015-2017

\section{CERTIFICATES}

- Exploratory Data Analysis (August 2016), Johns Hopkins University

- Getting and Cleaning Data (July 2016), Johns Hopkins University

- R Programming (June 2016), Johns Hopkins University

- Data Scientist's Toolbox (May 2016), Johns Hopkins University

- Applied Data Analysis Using SAS (Candidate), University of Wisconsin-Milwaukee

\section{NOTABLE COURSES}

- Advanced statistical analysis (Regression study, predictive models)

- Multivariate techniques in management research (Data mining, machine learning)

- Advanced machine learning and pattern recognition

- Probability models for operations decisions

- Advanced Microeconomics

- Industrial Organization

- Microeconomics

- Pricing and revenue analysis

- Supply chain management (Inventory control, operation management)

- Non-linear optimization (Gradient methods)

- Advanced computational methods in operations research (IP, MIP, metaheuristic methods)

- Database management (Relational databases and SQL writing)

- SAS programming 